\title{
Gold(I)-Catalyzed Reactivity of Furan-ynes with N-Oxides: Synthesis of Substituted Dihydropyridinones and Pyranones
}

\author{
Stefano Nejrotti, * Francesco Marra, Emanuele Priola, Andrea Maranzana, and Cristina Prandi* \\ Cite This: J. Org. Chem. 2021, 86, 8295-8307 \\ Read Online
}

ABSTRACT: The reactivity of "furan-ynes" in combination with pyridine and quinoline $\mathrm{N}$-oxides in the presence of a $\mathrm{Au}(\mathrm{I})$ catalyst, has been studied, enabling the synthesis of three different heterocyclic scaffolds. Selective access to two out of the three possible products, a dihydropyridinone and a furan enone, has been achieved through the fine-tuning of the reaction conditions. The reactions proceed smoothly at room temperature and open-air, and were further extended to a broad substrate scope, thus affording functionalized dihydropyridinones and pyranones.

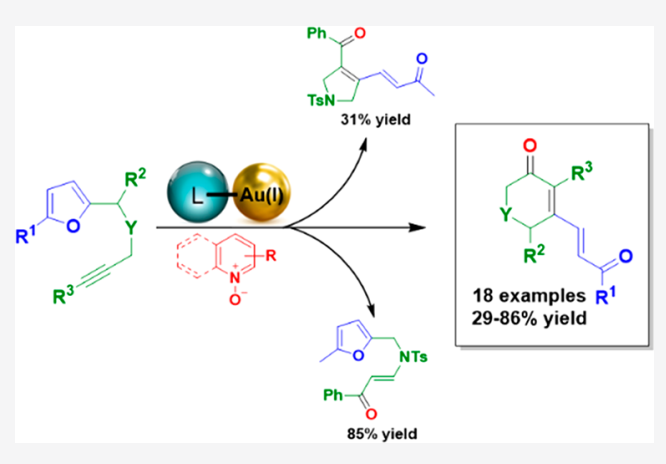

\section{INTRODUCTION}

The activation of alkynes toward the attack of a nucleophile by means of gold catalysis is a well-established tool in modern organic synthesis. ${ }^{1}$ Since the beginning of this century, the furan ring has emerged as a versatile candidate for both $\mathrm{Au}(\mathrm{I})$ - and $\mathrm{Au}(\mathrm{III})$-catalyzed transformations, by virtue of its nucleophilicity. ${ }^{2}$ A successful example in this regard is represented by the synthesis of phenols from furan-tethered alkynes ("furan-ynes"), which has been widely explored throughout the years, mainly by Hashmi and his group, enabling the synthesis of a variety of molecular scaffolds. ${ }^{3}$ More recently, the combination of furans with $\mathrm{Au}(\mathrm{I})$ carbene chemistry has disclosed intriguing synthetic possibilities. In 2014, Echavarren reported that three different types of $\mathrm{Au}(\mathrm{I})$ carbenes, generated respectively by rearrangement of propargyl esters, cycloisomerization of 1,6-enynes, and retro-Buchner reaction, underwent intermolecular reaction with furans through related mechanistic pathways, thus giving access to an array of diverse molecular frames (Scheme 1a). ${ }^{4}$ Gold(I) carbenes obtained from propargyl esters were later employed by Tang and Shi in intramolecular reactions with furans, achieving the synthesis of functionalized $\mathrm{N}$-heterocycles and $\mathrm{O}$-bridged tricyclic scaffolds (Scheme 1b). ${ }^{5}$ Another relevant class of Au(I) carbenes is represented by $\alpha$-oxo gold(I) carbenes. These reactive intermediates can be accessed by treatment of an alkyne with either a pyridine or quinoline $\mathrm{N}$-oxide in the presence of a $\mathrm{Au}(\mathrm{I})$ complex, through nucleophilic attack of the $\mathrm{O}$ atom of the $\mathrm{N}$-oxide onto the gold-activated triple bond and subsequent cleavage of the pyridine or quinoline (Scheme 1c). ${ }^{6}$

$\alpha$-Oxo gold(I) carbenes can undergo several reaction pathways, including cyclopropanations, migrations, ring expansions, insertions, and reactions with nucleophiles.

On these premises, following our interest for the synthetic manipulation of heterocycles through gold catalysis, ${ }^{8}$ we
Scheme 1. Background of the Work
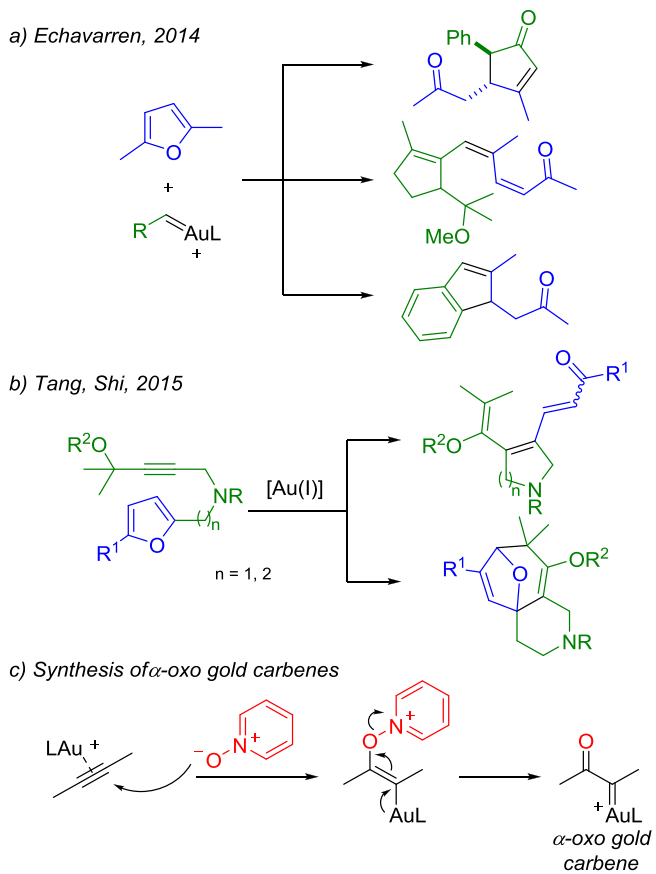

Received: March 31, 2021

Published: June 8, 2021 
Table 1. Optimization of the Reaction Conditions ${ }^{a}$

\begin{tabular}{|c|c|c|c|c|c|c|}
\hline \multirow[b]{2}{*}{ entry } & \multirow[b]{2}{*}[\mathrm{Au}]{} & \multirow[b]{2}{*}{ Ox } & \multirow[b]{2}{*}{ time } & \multicolumn{3}{|c|}{ yield $\%^{b}$} \\
\hline & & & & $\mathbf{2 a}(E / Z)$ & $3 \mathbf{a}(E / Z)$ & $4 a$ \\
\hline 1 & {$\left[(\mathrm{IPr}) \mathrm{Au}\left(\mathrm{NTf}_{2}\right)\right]$} & A & $6 \mathrm{~h}$ & $81(12 / 88)$ & - & - \\
\hline 2 & {$\left[\left(\left(2,4-{ }^{t} \mathrm{Bu}_{2} \mathrm{C}_{6} \mathrm{H}_{3} \mathrm{O}\right)_{3} \mathrm{P}\right) \mathrm{AuCl}\right] / \mathrm{AgNTf}_{2}$} & A & $6 \mathrm{~h}$ & $15(0 / 100)$ & $6(0 / 100)$ & 11 \\
\hline 3 & {$\left[\left(\left(p-\mathrm{CF}_{3} \mathrm{Ph}\right)_{3} \mathrm{P}\right) \mathrm{AuCl}\right] / \mathrm{AgNTf}_{2}$} & $\mathbf{A}$ & $6 \mathrm{~h}$ & $23(0 / 100)$ & $6(0 / 100)$ & 8 \\
\hline 4 & [(JohnPhos)AuCl]/AgNTf $f_{2}$ & A & $6 \mathrm{~h}$ & $77(6 / 94)$ & - & - \\
\hline 5 & {$[(\mathrm{JohnPhos}) \mathrm{Au}(\mathrm{NCMe})] \mathrm{SbF}_{6}$} & A & $6 \mathrm{~h}$ & $40(25 / 75)$ & - & - \\
\hline 6 & {$\left[\left({ }^{t} \mathrm{BuXPhos}\right) \mathrm{AuCl}\right] / \mathrm{AgNTf}_{2}$} & A & $6 \mathrm{~h}$ & $67(10 / 90)$ & - & - \\
\hline 7 & {$\left[(\mathrm{IPr}) \mathrm{Au}\left(\mathrm{NTf}_{2}\right)\right]$} & B & $6 \mathrm{~h}$ & - & $31(0 / 100)$ & 60 \\
\hline 8 & {$\left[(\mathrm{IPr}) \mathrm{Au}\left(\mathrm{NTf}_{2}\right)\right]$} & $\mathrm{C}$ & $6 \mathrm{~h}$ & $48(10 / 90)$ & - & - \\
\hline 9 & {$\left[(\mathrm{IPr}) \mathrm{Au}\left(\mathrm{NTf}_{2}\right)\right]$} & $\mathbf{D}$ & $6 \mathrm{~h}$ & $56(14 / 86)$ & $17(0 / 100)$ & 27 \\
\hline 10 & {$\left[(\mathrm{IPr}) \mathrm{Au}\left(\mathrm{NTf}_{2}\right)\right]$} & $\mathbf{E}$ & $6 \mathrm{~h}$ & - & - & 22 \\
\hline 11 & {$\left[(\mathrm{IPr}) \mathrm{Au}\left(\mathrm{NTf}_{2}\right)\right]$} & $\mathbf{F}$ & $6 \mathrm{~h}$ & $68(1 / 99)$ & - & - \\
\hline 12 & {$\left[(\mathrm{IPr}) \mathrm{Au}\left(\mathrm{NTf}_{2}\right)\right]$} & G & $6 \mathrm{~h}$ & - & - & - \\
\hline 13 & {$\left[(\mathrm{IPr}) \mathrm{Au}\left(\mathrm{NTf}_{2}\right)\right]$} & $\mathbf{F}$ & $20 \mathrm{~h}$ & $96(2 / 98)$ & - & - \\
\hline 14 & {$[($ MorDalPhos $) \mathrm{Au}(\mathrm{NCMe})] \mathrm{SbF}_{6}$} & B & $20 \mathrm{~h}$ & - & $4(0 / 100)$ & $85^{c}$ \\
\hline 15 & {$\left[\left(\left(2,4-{ }^{t} \mathrm{Bu}_{2} \mathrm{C}_{6} \mathrm{H}_{3} \mathrm{O}\right)_{3} \mathrm{P}\right) \mathrm{AuCl}\right] / \mathrm{AgNTf}_{2}$} & B & $20 \mathrm{~h}$ & - & $13(0 / 100)$ & 29 \\
\hline 16 & [(JohnPhos)AuCl] $/ \mathrm{AgNTf}_{2}$ & B & $20 \mathrm{~h}$ & - & $15(0 / 100)$ & 60 \\
\hline 17 & {$\left[\left(\left(\mathrm{C}_{6} \mathrm{~F}_{5}\right)_{3} \mathrm{P}\right) \mathrm{AuCl}\right] / \mathrm{AgNTf}_{2}$} & B & $20 \mathrm{~h}$ & - & $4(0 / 100)$ & 11 \\
\hline
\end{tabular}

${ }^{a}$ Conditions: $0.1 \mathrm{mmol}$ of $\mathbf{1 a}, 0.12 \mathrm{mmol}$ of $\mathrm{Ox}, 0.005 \mathrm{mmol}$ of $\mathrm{Au}(\mathrm{I})$ complex, and, when specified, $0.005 \mathrm{mmol}$ of Ag salt, in $1.0 \mathrm{~mL}$ of DCE. ${ }^{b}$ Determined by ${ }^{1} \mathrm{H}$ NMR with $n$-heptane as internal standard. ${ }^{c} 74 \%$ yield of isolated product.

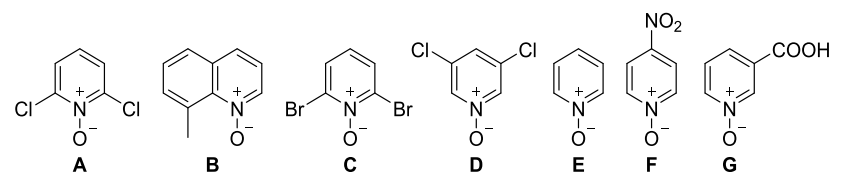

decided to investigate the reactivity of furan-ynes with $\mathrm{N}$-oxides, in the presence of a gold catalyst.

\section{RESULTS AND DISCUSSION}

To start the investigation, furan-yne 1a, provided with a phenyl terminus at the alkyne moiety, ${ }^{9}$ was reacted with the $\mathrm{Au}(\mathrm{I})$ complex $\left[(\mathrm{IPr}) \mathrm{Au}\left(\mathrm{NTf}_{2}\right)\right]$ and 2,6-dichlopyridine $\mathrm{N}$-oxide $\mathbf{A}$, at room temperature in 1,2-dichloroethane (Table 1 , entry 1 ). Under these conditions, the complete consumption of the starting material and the formation of the 6-membered dihydropyridinone $2 \mathrm{a}$ in $81 \%$ yield were observed after $6 \mathrm{~h}$. The structure of $2 \mathrm{a}$ was unequivocally identified through $\mathrm{X}$-ray diffraction. ${ }^{10}$ On the other hand, when $\left[\left(\left(2,4-{ }^{t} \mathrm{Bu}_{2} \mathrm{C}_{6} \mathrm{H}_{3} \mathrm{O}\right)_{3} \mathrm{P}\right)\right.$ $\mathrm{AuCl}]$ was used as the $\mathrm{Au}(\mathrm{I})$ complex, in combination with $\mathrm{AgNTf}_{2}$, the formation of two other products was observed: the 5-membered dihydropyrrole $3 a$ and the furan enone $4 a$, whose structures were again confirmed by XRD. ${ }^{10}$ However, in this case the yields of all three products were quite low (entry 2).

With the aim of optimizing and driving the selectivity of the reaction, several conditions and gold complexes were tested (entries 3-6; see Table S1 in the Supporting Information (SI) for the complete list of experiments), but none of them outdid $\left[(\mathrm{IPr}) \mathrm{Au}\left(\mathrm{NTf}_{2}\right)\right]$ in terms of the yield of $\mathbf{2 a}$. It should also be noted that the reaction proceeded with good stereoselectivity for the configuration of the exocyclic $\mathrm{C}-\mathrm{C}$ double bond in $\mathbf{2 a}$ and 3a, observing a general preference for the $Z$ isomer. With the best performing $\mathrm{Au}(\mathrm{I})$ complex, [(IPr) $\left.\mathrm{Au}\left(\mathrm{NTf}_{2}\right)\right]$, we shifted our attention to a screening of $\mathrm{N}$-oxides (entries 7-12), and we found that the selectivity of the reaction was sensitively affected by the choice of this reagent. With 8-methylquinoline $\mathrm{N}$-oxide $\mathbf{B}$, the formation of $2 \mathbf{a}$ was completely suppressed in favor of a $1 / 2$ ratio of $3 \mathbf{a}$ and $4 \mathbf{a}$ (entry 7$)$. Other $N$-oxides were either less selective (D, entry 9 ) or less active (entries 8, 10-12). However, 4-nitropyridine $\mathrm{N}$-oxide $\mathbf{F}$ afforded $\mathbf{2 a}$ with a remarkably high $Z$ selectivity (entry 11). Eventually, we identified the most suitable conditions for the synthesis of $2 \mathbf{a}$ with oxidant $\mathbf{F}$, by extending the reaction time up to $20 \mathrm{~h}$ (entry 13 ).

We then considered the outcome of the reaction with $\mathbf{B}$ and $\left[(\mathrm{IPr}) \mathrm{Au}\left(\mathrm{NTf}_{2}\right)\right]$ (entry 7), and we deemed it worthwhile to further explore the reactivity of this oxidant with different $\mathrm{Au}(\mathrm{I})$ complexes. With [(MorDalPhos) $\mathrm{Au}(\mathrm{NCMe})] \mathrm{SbF}_{6}$ the selective synthesis of the furan enone $4 a$ was achieved (entry 14). Unfortunately, we were not able to increase the yield of $3 a$ beyond $31 \%$, which remained as the best result for this product (entry 7 ). With the conditions in entry 13 (Table 1), product 2a was isolated in good yields, however we found that the $Z$ isomer slowly isomerized into $(E)-2 a$ over time. On this basis, we decided to direct the reaction toward complete $E$ selectivity, by a further optimization of the conditions described in entry 13. As shown in Table 2, the first parameter we investigated was the 
Table 2. Optimization of the $E / Z$ Ratio in the Synthesis of $2 a^{a}$

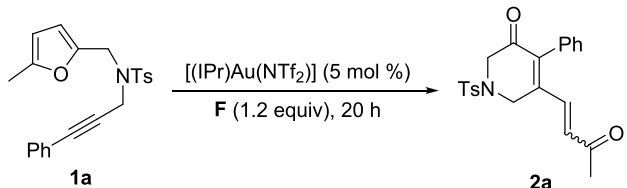

$1 \mathrm{a}$

\begin{tabular}{cllll}
\hline entry & $T\left({ }^{\circ} \mathrm{C}\right)$ & solvent & \multicolumn{1}{c}{ additive } & yield $\%^{b}(E / Z)$ \\
1 & $80{ }^{\circ} \mathrm{C}$ & $\mathrm{DCE}$ & - & $66(23 / 77)$ \\
2 & $\mathrm{rt}$ & $\mathrm{DCE}$ & $\mathrm{TFA}(2.0$ equiv $)$ & $73(16 / 84)$ \\
3 & $\mathrm{rt}$ & $\mathrm{DCE}$ & $p$-TsOH $(2.0$ equiv $)$ & $43(100 / 0)$ \\
4 & $\mathrm{rt}$ & $\mathrm{DCE}$ & $\mathrm{MsOH}(2.0$ equiv $)$ & $58(100 / 0)$ \\
5 & $\mathrm{rt}$ & $\mathrm{CHCl}$ & - & $63(3 / 97)$ \\
6 & $\mathrm{rt}$ & $\mathrm{HFIP}$ & - & - \\
7 & $\mathrm{rt}$ & $\mathrm{DCE}$ & $p-\mathrm{TsOH}(2.0 \text { equiv })^{c}$ & $89(11 / 89)$ \\
8 & $\mathrm{rt}$ & $\mathrm{DCE}$ & $\mathrm{MsOH}(2.0 \text { equiv })^{c}$ & $94(17 / 83)$ \\
9 & $\mathrm{rt}$ & $\mathrm{DCE}$ & $\mathrm{MsOH}(5.0 \text { equiv })^{c}$ & $80^{d}(100 / 0)$
\end{tabular}

${ }^{a}$ TFA $=$ trifluoroacetic acid; HFIP $=$ hexafluoroisopropanol. ${ }^{b}$ Determined by ${ }^{1} \mathrm{H}$ NMR with $n$-heptane as internal standard. ${ }^{c}$ Added after $20 \mathrm{~h}$, then stirred for one additional hour. ${ }^{d} 72 \%$ yield of isolated product.

temperature, which was raised to $80{ }^{\circ} \mathrm{C}$ (Table 2 , entry 1$)$. This resulted indeed in an improvement of the $E / Z$ ratio, but with a decrease in the overall yield. A different approach, based on the addition of a Brønsted acid as promoter of the isomerization, afforded (E)-2a with complete selectivity, but the yields were again not satisfactory ( $p$-toluenesulfonic acid, $p$ - $\mathrm{TsOH}$, and methanesulfonic acid, $\mathrm{MsOH}$, entries 3 and 4). Also attempts to perform the reaction in more acidic solvents, such as chloroform and hexafuoroisopropanol (entries 5 and 6), were met with failure. The decrease in the yield may be attributed to the possible degradation of the starting material $\mathbf{1 a}$ in the presence of a Brønsted acid. On this basis, we decided to add the acid only at the end of the $\mathrm{Au}(\mathrm{I})$-catalyzed step, i.e. after $20 \mathrm{~h}$ (entries 79): under these conditions, with $\mathrm{MsOH}$ a remarkable $80 \% \mathrm{NMR}$ yield of $(E)-\mathbf{2 a}$ (72\% after isolation) was obtained by adjusting the amount of $\mathrm{MsOH}$ to 5.0 equiv (entry 9; see Table $\mathrm{S} 2$ in the SI for the complete list of experiments).

Having identified the most suitable conditions to orient the divergent reactivity of $\mathbf{1 a}$ toward the exclusive formation of dihydropyridinone $\mathbf{2 a}$ as the $E$ isomer, we investigated the possible extension of the reaction substrate scope. Furan-ynes $\mathbf{1 b}-\mathbf{r}$ were synthesized and reacted with 4-nitropyridine $\mathrm{N}$ oxide $\mathbf{F}$ and $\left[(\operatorname{IPr}) \mathrm{Au}\left(\mathrm{NTf}_{2}\right)\right]$ for $20 \mathrm{~h}$, after which 5.0 equiv of $\mathrm{MsOH}$ were added, and the mixture was stirred for one additional hour (Scheme 2). Variations on the terminal aromatic ring were introduced, proving that a variety of electron-donating $(\mathbf{2} \mathbf{b}-\mathbf{d})$ and electron-withdrawing $(\mathbf{2} \mathbf{e}-\mathbf{g})$ functional groups are well tolerated. Apart from substituted phenyl rings, thiophene and naphthalene were also suitable $(\mathbf{2} \mathbf{h}-\mathbf{i})$. Conversely, in the presence of an alkyl chain $\left(\mathrm{R}^{3}=n-\mathrm{Bu}\right)$ the reaction did not occur, and degradation of the starting material was observed. The methyl group on the furan ring in the starting material was replaced with phenyl, thienyl, and furyl groups, obtaining aryl ketones $\mathbf{2 j}-\mathbf{m}$ as product. Unfortunately, with $\mathrm{R}^{1}=\mathrm{H}(\mathbf{1} \mathbf{s}$; see the SI), the reaction was troublesome and hardly reproducible. On the other hand, replacing the $N$-Ts linker with an $O$ linker resulted in the synthesis of pyranones $\mathbf{2 n - p}$. Substituents on the heterocyclic ring were also successfully introduced, affording pyranones $\mathbf{2 q}-\mathbf{r}$. In all cases, apart from product $\mathbf{2 p}$, complete $E$ selectivity was observed, and the yields ranged from moderate to high.
Scheme 2. Substrate Scope for the Synthesis of Dihydropyridinones and Pyranones ${ }^{a}$
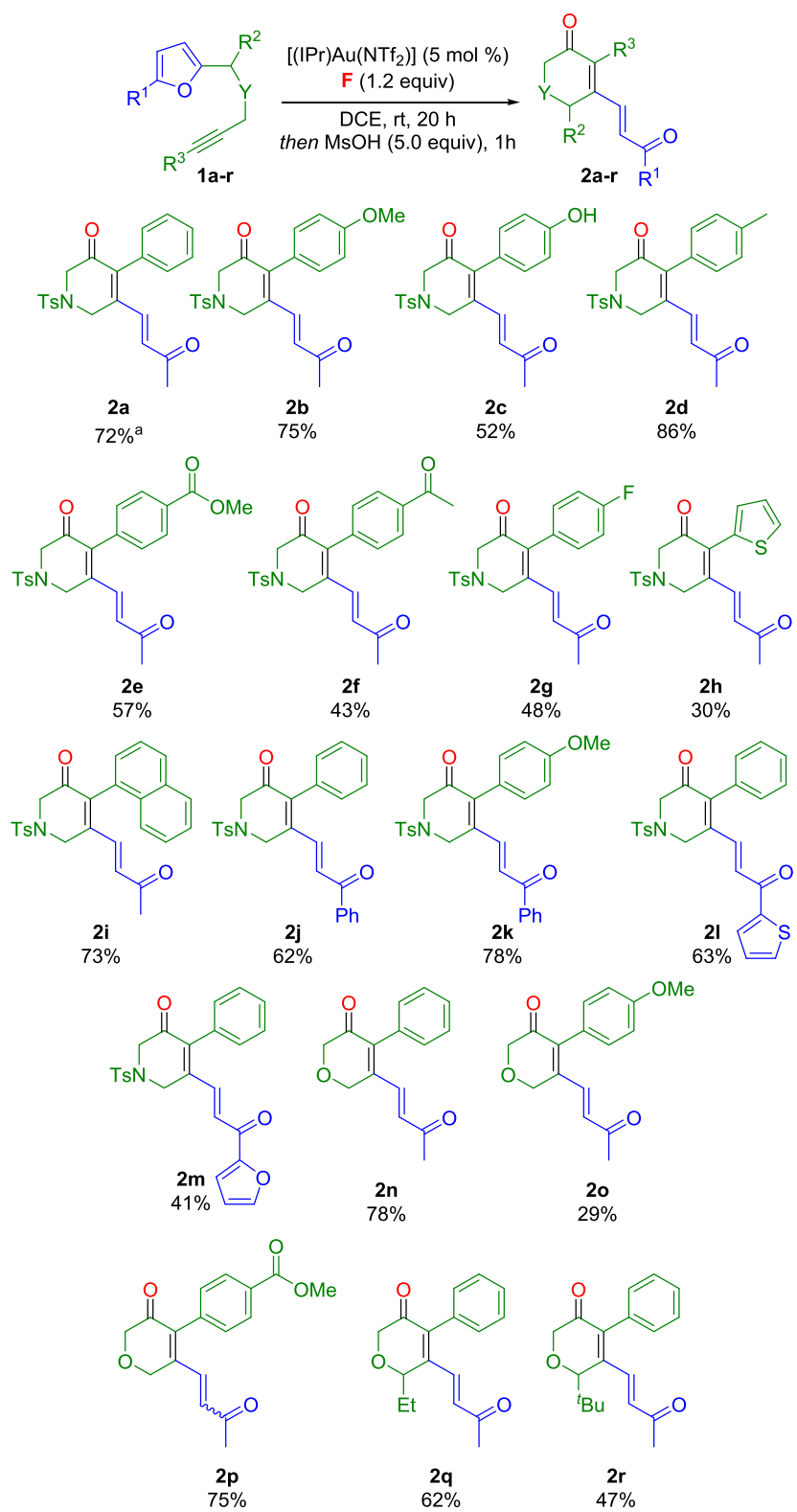

$\mathrm{Me}$

$29 \%$
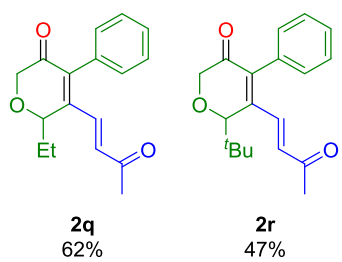

${ }^{a}$ Yield unchanged at $1.3 \mathrm{mmol}$ scale. ${ }^{a}$ Reactions performed at a 0.2 mmol scale. Yields of isolated products. $E / Z$ ratio $100 / 0$ in all cases, except for $2 \mathbf{p}(E / Z 40 / 60)$.

A plausible mechanistic picture for the formation of products $\mathbf{2 a}, \mathbf{3 a}$, and $\mathbf{4 a}$ from furan-yne $\mathbf{1 a}$ is depicted in Scheme 3.,6,11 Upon activation of the alkyne moiety in substrate 1 a by the $\mathrm{Au}(\mathrm{I})$ complex, the attack of the $\mathrm{N}$-oxide onto the $\mathrm{C} 1$ atom of the triple bond leads to $\alpha$-oxo gold(I) carbene I (see Scheme 1c), which then undergoes cyclization through nucleophilic attack by the furan ring, affording the spirocyclic intermediate II (path a). A cyclopropanation/cyclopropane opening sequence eventually determines the opening of the furan ring, leading to the formation of 2a. Intermediate II might also convert directly to $2 \mathrm{a}$ through an elimination step. Based on the results presented in Tables 1 and 2, the $Z$ isomer is likely first formed and then converted into $(E)-2 \mathbf{a} .{ }^{12}$ If the regioselectivity of the $N$-oxide attack is switched, the spirocyclic intermediate $\mathbf{V}$ is formed upon attack of the furan onto $\mathrm{Au}(\mathrm{I})$ carbene IV (path b). Then, the 
Scheme 3. Mechanistic Pathways for 2a, 3a, and 4a

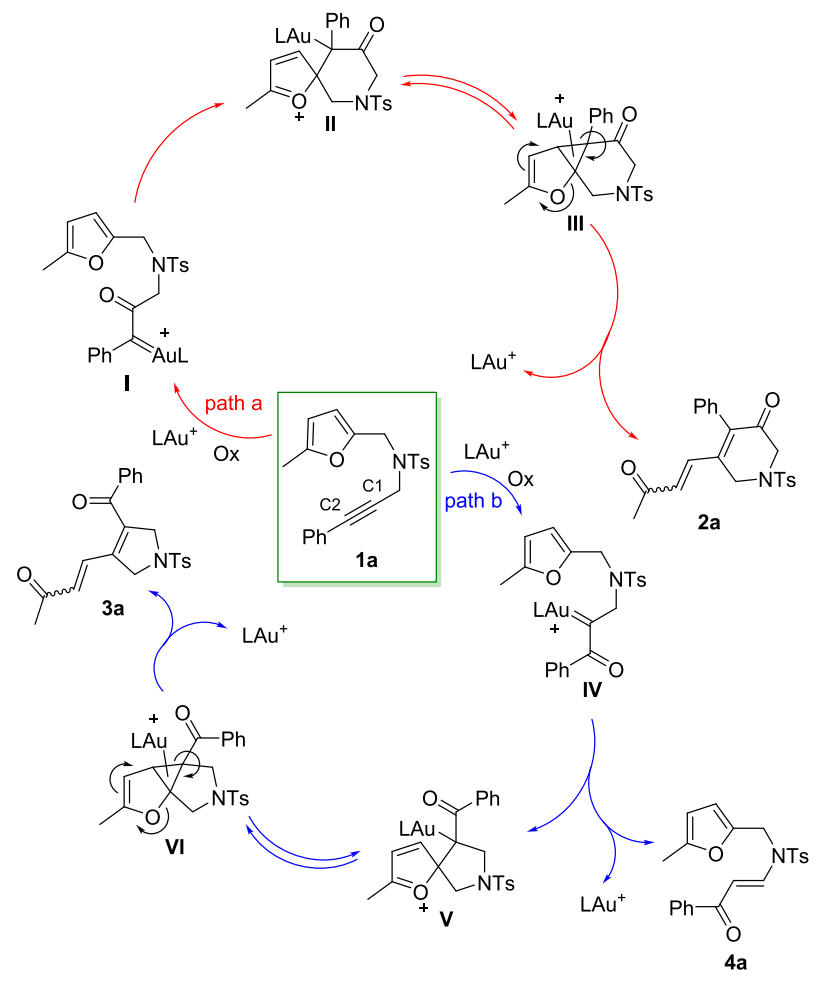

final product $3 \mathbf{a}$ is obtained through a mechanism analogous to the one described for 2a. Alternatively, the $\alpha$-oxo gold(I) carbene intermediate IV may also undergo a 1,2-H shift with the neighboring $\mathrm{CH}_{2}$ group, leading to the formation of furan enone 4a. ${ }^{8 c, 13}$

It should be pointed out that the best selectivity for $\mathbf{4 a}$ was obtained with the gold(I) complex [(MorDalPhos)Au$(\mathrm{NCMe})] \mathrm{SbF}_{6}$ (Table 1, entry 14). The MorDalPhos ligand has been reported to temper the electrophilicity of the $\mathrm{Au}(\mathrm{I})$ carbene through bidentate coordination from both the $\mathrm{P}$ and the $\mathrm{N}$ atom. ${ }^{14}$ This would be consistent what was observed in our case, as the less electrophilic carbene is likely less prone to get attacked by the nucleophilic furan ring, and thus more available to selectively undergo a 1,2-H shift. However, when more electrophilic $\mathrm{Au}(\mathrm{I})$ complexes were employed, the expected enhancement in the selectivity toward 3a was not observed (Table 1, entries 15 and 17).

As highlighted by the study on the reaction conditions (Table 1 ), the $\mathrm{C} 1$ vs $\mathrm{C} 2$ regioselectivity of the nucleophilic attack is highly dependent on the nature of the $\mathrm{N}$-oxide reagent, while the electronic effects of the alkyne substituent $\mathrm{R}^{3}$ are not relevant in modifying this selectivity (Scheme 2 ). To better elucidate the influence of the electronic and steric factors, we studied the attack of $N$-oxides $\mathbf{F}$ and $\mathbf{B}$ onto the $[\mathrm{LAu}]^{+}-\mathbf{1 a} \pi$-complex through DFT calculation (Table 3). The Natural Bond Analysis of this intermediate revealed that the charge density values on $\mathrm{C} 1$ and $\mathrm{C} 2$ are very small, being slightly negative on $\mathrm{C} 1(-0.10)$ and slightly positive on $\mathrm{C} 2(+0.04)$. On the other hand, the partial negative charge on the $\mathrm{O}$ atom in the $\mathrm{N}$-oxide is larger in $\mathbf{B}(-0.55)$ than in $\mathbf{F}(-0.49)$. By considering the charge density only, $\mathbf{B}$ would be expected to be more reactive than $\mathbf{F}$ and $\mathrm{C} 2$ would be the preferential site of reaction. Thus, a more detailed study on the transition states for the attack of $\mathbf{B}$ and $\mathbf{F}$ onto $\mathbf{C 1}$ and $\mathrm{C} 2$ was carried out, and the results are reported in Table 3. The addition of $\mathbf{B}$ to $\mathrm{C} 1$ is preferred to the addition to $\mathrm{C} 2$ : the
Table 3. Free Energy Barriers and Energy Decomposition Analysis, ${ }^{a}$ in $\mathrm{kcal} \mathrm{mol}^{-1}$, for the Addition of B and F to $\mathrm{Cl}$ and C2

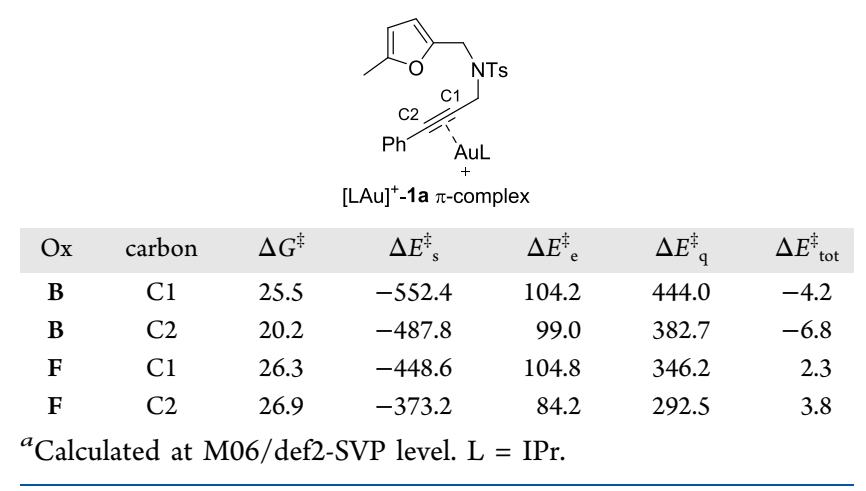

free energy barriers $\left(\Delta G^{\ddagger}\right)$ are 20.2 and $25.5 \mathrm{kcal} \mathrm{mol}^{-1}$, respectively. On the other hand, $\mathbf{F}$ displays opposite behavior, with a smaller barrier for the addition to $\mathrm{C} 1\left(26.3 \mathrm{kcal} \mathrm{mol}^{-1}\right)$. B appears to be more reactive than $F$. To explain the regioselectivity, Shubin Liu's energy decomposition analysis (EDA-SBL) was carried out. The EDA-SBL method splits the total energy $\left(E_{\text {tot }}\right)$ in three components: $E_{\mathrm{e}}$ (sum of nuclearnuclear, electron-electron, and nuclear-electron electrostatic interactions), $E_{\mathrm{q}}$ (quantum interaction: sum of exchangecorrelation and Pauli energies, associated to the repulsion between filled orbitals), and $E_{\mathrm{s}}$ (steric energy repulsion). The larger values of the three components indicate the main effects responsible for the barriers. In all the four reactions, the barriers are dominated by change of steric $\left(\Delta E_{\mathrm{s}}^{\ddagger}\right)$ and quantum $\left(\Delta E_{\mathrm{q}}^{\ddagger}\right)$ effects. The quantum effect contributes positively to the height of the barrier, and the steric effect, negatively. Electrostatic effects $\left(\Delta E_{\mathrm{e}}^{\ddagger}\right)$ are relevant but less important. By comparing the $\Delta E^{\ddagger}{ }_{s}$ for $\mathrm{C} 1$ and $\mathrm{C} 2$, the addition to the former displays less negative values. The difference $\Delta E^{\ddagger}(\mathrm{C} 2)-\Delta E^{\ddagger}{ }_{s}(\mathrm{C} 1)$ is 64.4 and 75.4 for $\mathbf{B}$ and $\mathbf{F}$ respectively, which could indicate a larger steric effect when addition occurs to $\mathrm{C} 2$. The regioselectivity is supposed to depend on a delicate balance between density of charge on the oxidant, responsible for the positive contribution to the barrier, and steric effect. By comparison, $N$-oxide $\mathbf{D}$ shows a charge density on the $\mathrm{O}$ atom of -0.52 , intermediate between $\mathbf{B}$ and F, and its attack on both C1 and C2 (Table 1, entry 9). Noncovalent interaction plots (Figure S5 in the SI) visualize noncovalent interactions from the topological analysis of the electron density. These highlight the presence of van der Waals attractive interactions between the phenyl group and the pyridine or quinoline rings, while strong steric repulsion between the $N$-oxide and the reactant are lacking in the transition structures.

To provide a glimpse of the synthetic value of the products of our $\mathrm{Au}(\mathrm{I})$-catalyzed methodology, we subjected $\mathbf{2 a}$ to the synthetic manipulations reported in Scheme 4. Remarkably, the Michael addition of thiophenol, catalyzed by $\mathrm{Et}_{3} \mathrm{~N},{ }^{15}$ proceeded smoothly at room temperature with complete selectivity for the exocyclic double bond over the endocyclic one, thus obtaining sulfide 5. Similarly to the Michael addition, also the hydrogenation over $\mathrm{Pd} / \mathrm{C}$ was selective for the exocyclic double bond, affording the partially saturated compound 6. On the other hand, reduction with $\mathrm{NaBH}_{4}$ did not discriminate between the two carbonyl groups, and diol 7 was obtained as the product. 


\section{Scheme 4. Synthetic Manipulations of 2a}

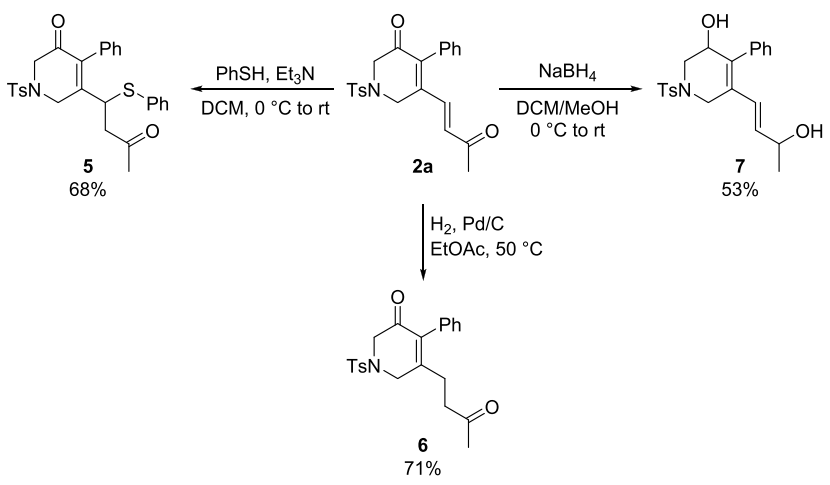

\section{CONCLUSION}

In conclusion, the study of the reactivity of furan-ynes with $\mathrm{N}$ oxides, in the presence of a $\mathrm{Au}(\mathrm{I})$ catalyst, has disclosed an intriguing divergent mechanistic picture, with the formation of three possible densely functionalized heterocyclic scaffolds. This work contributes to extend the wide landscape of synthetic opportunities offered by the powerful combination of gold catalysis and furans, enabling the straightforward synthesis of dihydropyridinones and pyranones through control over the product selectivity and the diastereoselectivity of the reaction. Moreover, the good tolerance of structural variations in the substrate, as well as of the presence of either nucleophilic or electrophilic functional groups, allows for a tailored application of the methodology, in view of its exploitation in synthesis. The theoretical investigation on the regioselectivity contributes to the understanding of the role of electronic and steric effects in the mechanism of this class of reactions, and could potentially prompt a systematic more-in-detail analysis of previously reported similar methodologies.

\section{EXPERIMENTAL SECTION}

Computational Method. The stationary points were optimized in the gas phase with the DFT M06 functional, ${ }^{16}$ with the ONIOM procedure. ${ }^{17}$ In this procedure, the molecule is divided into two layers treated with different levels of calculations. This approach has been found to be useful for modeling large molecular systems. In this work, the higher layer was treated at the M06/def2-SVP ${ }^{18}$ level, whereas for the lower layer the chosen level was M06/STO-3G ${ }^{19}$ (for Au only, effective core potential LanL2DZ was used). This procedure was labeled as ONIOM(M06/def2-SVP:M06/STO-3G). The figures showing the layers assignment of the atoms are reported in the Supporting Information (Figure S4). The energies were refined by single-point energy calculations at level ONIOM(M06/def2TZVP:M06/STO-3G) including the solvent effect (dichloroethane) by the Solvation Model based on Density (SMD). ${ }^{20}$ The relative Gibbs free energies in solution $(\Delta G)$ were estimated at $T=298 \mathrm{~K}$. Multiwfn software ${ }^{21}$ was used for the noncovalent interaction analysis $(\mathrm{NCI})^{22}$ and for Shubin Liu's energy decomposition analysis (EDA-SBL) ${ }^{23}$ Geometry optimizations and thermochemistry calculations were carried out by using the Gaussian 16 programs.

General Information. Flasks and all equipment used for the generation and reaction of moisture-sensitive compounds were dried by electric heat gun under nitrogen. Reaction heating was provided through an oil bath. Reagents and solvents were purchased from Merck, Acros Organics, TCI or Alfa Aesar. Anhydrous THF was obtained by distillation over $\mathrm{LiAlH}_{4}$, followed by distillation over Na-benzophenone; $\mathrm{Et}_{3} \mathrm{~N}$ was distilled over $\mathrm{CaH}_{2}$. All other reagents were used as received, without further purification. Flash column chromatography was performed over silica gel (40-63 $\mu \mathrm{m}, 230-400 \mathrm{mesh}) ; R_{f}$ values refer to TLC carried out on silica gel plates. ${ }^{1} \mathrm{H}$ NMR and ${ }^{13} \mathrm{C}$ NMR spectra were recorded on a Jeol ECZR600, in $\mathrm{CDCl}_{3}$ or in $\mathrm{CD}_{3} \mathrm{OD}$, using the residual solvent peak as an internal reference $\left(\mathrm{CHCl}_{3},{ }^{1} \mathrm{H}\right.$ : 7.26 ppm; $\mathrm{CDCl}_{3},{ }^{13} \mathrm{C}: 77.16$ ppm; $\mathrm{CH}_{3} \mathrm{OH},{ }^{1} \mathrm{H}: 3.34$ ppm; $\mathrm{CD}_{3} \mathrm{OD}$, ${ }^{13} \mathrm{C}$ : $\left.49.86 \mathrm{ppm}\right)$. Multiplicity is reported as follows: s (singlet), $\mathrm{d}$ (doublet), t (triplet), q (quartet), m (multiplet), br (broad). GC-MS spectra were recorded at an ionizing voltage of $70 \mathrm{eV}$. HRMS analysis were run on a high resolving power hybrid mass spectrometer (HRMS) Orbitrap Fusion (Thermo Scientific, Rodano, Italy), equipped with an a ESI ion source. The samples were analyzed in acetonitrile solution using a syringe pump at a flow rate of $5 \mu \mathrm{L} / \mathrm{min}$. The tuning parameters adopted for the ESI source were as follows: source voltage $5.0 \mathrm{kV}$, source current $0.5 \mu \mathrm{A}$, capillary voltage $32 \mathrm{~V}$, tube lens voltage $75 \mathrm{~V}$. The heated capillary temperature was maintained at $275^{\circ} \mathrm{C}$. Crystals of 2a, 3a, and 4a were obtained by slow evaporation from a $\mathrm{MeCN}$ solution at 4-6 ${ }^{\circ} \mathrm{C}$. Reaction schemes for the synthesis of the substrates, as well as details about XRD analysis, are available in the Supporting Information.

Synthesis of Furan-2-ylboronic Acid, int-1. This is a modified literature procedure. ${ }^{24} \mathrm{~A}$ solution of furan (1.0 equiv, $20 \mathrm{mmol}$ ) in anhydrous THF $(1.0 \mathrm{M})$, under a $\mathrm{N}_{2}$ atmosphere, was cooled down to $-10{ }^{\circ} \mathrm{C} . n$-BuLi (1.0 equiv, $2.5 \mathrm{M}$ solution in hexane) was added, and the mixture was allowed to warm to $0{ }^{\circ} \mathrm{C}$ and stirred for $1 \mathrm{~h}$. Then, triisopropylborate (2.0 equiv) was added, and the mixture was allowed to warm to room temperature and stirred for $30 \mathrm{~min}$, before addition of $50 \mathrm{~mL}$ of a $3 \mathrm{M} \mathrm{HCl}$ solution. The mixture was extracted three times with $\mathrm{Et}_{2} \mathrm{O}$, and the combined organic layers were extracted three times with a $1 \mathrm{M} \mathrm{NaOH}$ solution. The combined $\mathrm{NaOH}$ acqueous layers were brought to acidic $\mathrm{pH}$ with a $6 \mathrm{M} \mathrm{HCl}$ solution and extracted three times with $\mathrm{Et}_{2} \mathrm{O}$; the combined organic layers were dried over anhydrous $\mathrm{Na}_{2} \mathrm{SO}_{4}$ and filtered, and the volatiles wee evaporated under reduced pressure to afford furan-2-ylboronic acid as a yellow solid, which was used for the next step without further purification $(1.320 \mathrm{~g}, 59 \%$ yield). ${ }^{1} \mathrm{H}$ NMR (600 MHz, dmso-d6) $\delta$ (ppm): 8.21 (br s, 2H), 7.84 (d, $1 \mathrm{H}, J$ $=1.7 \mathrm{~Hz}), 7.09(\mathrm{~d}, 1 \mathrm{H}, J=3.3 \mathrm{~Hz}), 6.50(\mathrm{dd}, 1 \mathrm{H}, J=3.3,1.7 \mathrm{~Hz})$.

General Procedure for the Suzuki Coupling of 5-Bromo-2furaldehyde (GP1). ${ }^{25}$ Into a flask, 2-bromofuradelhyde (1.0 equiv, 15 $\mathrm{mmol})$, arylboronic acid (1.1 equiv), palladium(II) acetate (0.02 equiv), potassium carbonate (2.5 equiv), and tetrabutylammonium bromide ( 1.0 equiv) were added, then deionized $\mathrm{H}_{2} \mathrm{O}(0.5 \mathrm{M}$ with respect to 2-bromofuraldehyde) was added, and the mixture was vigorously stirred for $2 \mathrm{~h}$ at room temperature. Then, the mixture was extracted three times with $\mathrm{EtOAc}$; the combined organic layers were dried over anhydrous $\mathrm{Na}_{2} \mathrm{SO}_{4}$ and filtered, and the volatiles were evaporated under reduced pressure. The crude product was purified by flash column chromatography to obtain the pure 5-aryl substituted furfural.

5-Phenylfuran-2-carbaldehyde, ${ }^{26}$ int-2. Synthesized according to the general procedure GP1. Yellow oil, $1.93 \mathrm{~g}, 75 \%$ yield. $R_{f} 0.46(9 / 1$ PE/EtOAc). ${ }^{1} \mathrm{H}$ NMR $\left(600 \mathrm{MHz}, \mathrm{CDCl}_{3}\right) \delta(\mathrm{ppm}): 9.66(\mathrm{~s}, 1 \mathrm{H})$, 7.85-7.82 (m, 2H), 7.47-7.44 (m, 2H), 7.42-7.39 (m, 1H), $7.33(\mathrm{~d}$, $1 \mathrm{H}, J=3.7 \mathrm{~Hz}), 6.85(\mathrm{~d}, 1 \mathrm{H}, J=3.7 \mathrm{~Hz})$. GC-MS $m / z(\%): 172[\mathrm{M}]^{+}$ (100), 171 (45), 115 (77).

5-(Thiophen-2-yl)furan-2-carbaldehyde, ${ }^{27}$ int-3. Synthesized according to the general procedure GP1. Yellow oil, $2.54 \mathrm{~g}, 95 \%$ yield. $R_{f}$ 0.46 (9/1 PE/EtOAc). ${ }^{1} \mathrm{H}$ NMR $\left(600 \mathrm{MHz}, \mathrm{CDCl}_{3}\right) \delta(\mathrm{ppm}): 9.62(\mathrm{~s}$, $1 \mathrm{H}), 7.53(\mathrm{dd}, 1 \mathrm{H}, J=3.7,1.2 \mathrm{~Hz}), 7.41(\mathrm{dd}, 1 \mathrm{H}, J=5.0,1.2 \mathrm{~Hz}), 7.29$ $(\mathrm{d}, 1 \mathrm{H}, J=3.8 \mathrm{~Hz}), 7.11(\mathrm{dd}, 1 \mathrm{H}, J=5.0,3.7 \mathrm{~Hz}), 6.68(\mathrm{~d}, 1 \mathrm{H}, J=3.7$ $\mathrm{Hz})$. GC-MS $m / z(\%): 178[\mathrm{M}]^{+}(100), 121$ (72).

$\left[2,2^{\prime}\right.$-Bifuran]-5-carbaldehyde, ${ }^{28}$ int-4. Synthesized according to the general procedure GP1. Yellow oil, $2.06 \mathrm{~g}, 85 \%$ yield. $R_{f} 0.36(9 / 1$ PE/EtOAc). ${ }^{1} \mathrm{H}$ NMR $\left(600 \mathrm{MHz}, \mathrm{CDCl}_{3}\right) \delta(\mathrm{ppm}): 9.62(\mathrm{~s}, 1 \mathrm{H}), 7.52$ $(\mathrm{dd}, 1 \mathrm{H}, J=1.8,0.8 \mathrm{~Hz}), 7.30(\mathrm{~d}, 1 \mathrm{H}, J=3.7 \mathrm{~Hz}), 6.91(\mathrm{dd}, 1 \mathrm{H}, J=3.4$, $0.8 \mathrm{~Hz}), 6.73(\mathrm{~d}, 1 \mathrm{H}, J=3.7 \mathrm{~Hz}), 6.53(\mathrm{dd}, 1 \mathrm{H}, J=3.5,1.8 \mathrm{~Hz})$. GC-MS $m / z(\%): 162[\mathrm{M}]^{+}(100), 105(65)$.

General Procedure for the Synthesis of Terminal Alkynes with $N$-Ts Linker (GP2). ${ }^{29}$ To a $1.0 \mathrm{M}$ solution of furaldehyde (1.0 equiv, $10-20 \mathrm{mmol}$ ) in $\mathrm{DCM} \mathrm{MgSO}_{4}$ (1.1 equiv) and propargylamine (1.0 equiv) were added, and the mixture was stirred at room temperature for 24-48 h (GC-MS monitoring). The mixture was filtered over silica with EtOAc, and the volatiles were evaporated under 
reduced pressure to obtain the crude imine, which was used for the next step without further purification.

To a $1.0 \mathrm{M}$ solution of the crude furanyl imine in $\mathrm{MeOH}, \mathrm{NaBH}_{4}$ (1.0 equiv) was added portionwise at $0{ }^{\circ} \mathrm{C}$. The mixture was allowed to reach room temperature and stirred for 5-10 min (GC-MS monitoring) until complete conversion. Then, $\mathrm{MeOH}$ was evaporated to half of the initial volume under reduced pressure. Water was added, and the mixture was extracted three times with EtOAc; the combined organic layers were dried over $\mathrm{Na}_{2} \mathrm{SO}_{4}$ and filtered, and the volatiles were evaporated under reduced pressure to obtain the crude amine, which was used for the next step without further purification.

To a $1.0 \mathrm{M}$ solution of the crude amine in $\mathrm{DCM}, \mathrm{Et}_{3} \mathrm{~N}$ (1.0 equiv) was added, then $p$-toluenesulfonyl chloride (1.0 equiv) was added portionwise, and the mixture was stirred at room temperature overnight. Then, water was added, and the mixture was extracted three times with DCM; the combined organic layers were dried over $\mathrm{Na}_{2} \mathrm{SO}_{4}$ and filtered, and the volatiles were evaporated under reduced pressure. The crude product was purified by flash column chromatography to obtain the pure $\mathrm{N}$-Ts product.

$N$-(Furan-2-ylmethyl)-4-methyl- $N$-(prop-2-yn-1-yl)benzenesulfonamide, ${ }^{30}$ int -5 . Synthesized according to the general procedure GP2. White solid, $1.46 \mathrm{~g}, 51 \%$ yield (3 steps). $R_{f} 0.47$ (80/ $15 / 5 \mathrm{PE} / \mathrm{DCM} / \mathrm{EtOAc}) .{ }^{1} \mathrm{H}$ NMR $\left(600 \mathrm{MHz}, \mathrm{CDCl}_{3}\right) \delta(\mathrm{ppm})$ : $7.75-7.73(\mathrm{~m}, 2 \mathrm{H}), 7.35(\mathrm{dd}, 1 \mathrm{H}, J=1.8,0.9 \mathrm{~Hz}), 7.31-7.28(\mathrm{~m}, 2 \mathrm{H})$, $6.31(\mathrm{dd}, 1 \mathrm{H}, J=3.3,1.8 \mathrm{~Hz})$ superimposed to $6.30(\mathrm{dd}, 1 \mathrm{H}, J=6.3,0.8$ $\mathrm{Hz}), 4.43(\mathrm{~s}, 2 \mathrm{H}), 4.02(\mathrm{~d}, 2 \mathrm{H}, J=2.5 \mathrm{~Hz}), 2.43(\mathrm{~s}, 3 \mathrm{H}), 2.07(\mathrm{t}, 1 \mathrm{H}, J=$ $2.5 \mathrm{~Hz}$ ). GC-MS $m / z(\%): 289[\mathrm{M}]^{+}(1), 134$ (100), $106(32), 91$ (31), 81 (17).

4-Methyl- $N$-((5-methylfuran-2-yl)methyl)- $N$-(prop-2-yn-1yl)benzenesulfonamide, ${ }^{29}$ int- 6 . Synthesized according to the general procedure GP2. White solid, $3.82 \mathrm{~g}$, $63 \%$ yield ( 3 steps). $R_{f} 0.31$ (9/1 PE/EtOAc). ${ }^{1} \mathrm{H}$ NMR $\left(600 \mathrm{MHz}, \mathrm{CDCl}_{3}\right) \delta(\mathrm{ppm}): 7.75-7.73$ $(\mathrm{m}, 2 \mathrm{H}), 7.30-7.28(\mathrm{~m}, 2 \mathrm{H}), 6.16(\mathrm{~d}, 1 \mathrm{H}, J=3.0 \mathrm{~Hz}), 5.86(\mathrm{dq}, 1 \mathrm{H}, J=$ $3.0 \mathrm{~Hz}, 1.0 \mathrm{~Hz}), 4.37(\mathrm{~s}, 2 \mathrm{H}), 4.02(\mathrm{~d}, 2 \mathrm{H}, J=2.5 \mathrm{~Hz}), 2.43(\mathrm{~s}, 3 \mathrm{H}), 2.20$ $(\mathrm{d}, 3 \mathrm{H}, J=1.0 \mathrm{~Hz}), 2.05(\mathrm{t}, 1 \mathrm{H}, J=2.5 \mathrm{~Hz}) . \mathrm{GC}-\mathrm{MS} m / z(\%): 303[\mathrm{M}]^{+}$ (3), 148 (100), 147 (36), 120 (77), 95 (82), 91 (42).

4-Methyl- $\mathrm{N}$-((5-phenylfuran-2-yl)methyl)- $\mathrm{N}$-(prop-2-yn-1yl)benzenesulfonamide, int-7. Synthesized according to the general procedure GP2. White solid, $2.33 \mathrm{~g}$, $64 \%$ yield (3 steps), $\mathrm{mp} 89-91{ }^{\circ} \mathrm{C}$ $\left(\mathrm{Et}_{2} \mathrm{O}\right) . R_{f} 0.38$ (9/1 PE/EtOAc). ${ }^{1} \mathrm{H}$ NMR $\left(600 \mathrm{MHz}, \mathrm{CDCl}_{3}\right) \delta$ (ppm): 7.76-7.74 (m, 2H), 7.56-7.54 (m, 2H), 7.37-7.34 (m, 2H), $7.28-7.24(\mathrm{~m}, 3 \mathrm{H}), 6.55(\mathrm{~d}, 1 \mathrm{H}, J=3.3 \mathrm{~Hz}), 6.37(\mathrm{~d}, 1 \mathrm{H}, J=3.3 \mathrm{~Hz})$, $4.52(\mathrm{~s}, 2 \mathrm{H}), 4.09(\mathrm{~d}, 2 \mathrm{H}, J=2.5 \mathrm{~Hz}), 2.38(\mathrm{~s}, 3 \mathrm{H}), 2.11(\mathrm{t}, 1 \mathrm{H}, J=2.5$ $\mathrm{Hz}) .{ }^{13} \mathrm{C}\left\{{ }^{1} \mathrm{H}\right\} \operatorname{NMR}\left(150 \mathrm{MHz}, \mathrm{CDCl}_{3}\right) \delta(\mathrm{ppm}): 154.5(\mathrm{Cq}), 148.2$ (Cq), $143.8(\mathrm{Cq}), 136.2(\mathrm{Cq}), 130.5(\mathrm{Cq}), 129.7(\mathrm{CH}), 128.7(\mathrm{CH})$, $127.8(\mathrm{CH}), 127.7(\mathrm{CH}), 123.9(\mathrm{CH}), 112.3(\mathrm{CH}), 105.7(\mathrm{CH}), 76.7$ (Cq), $74.1(\mathrm{CH}), 43.1\left(\mathrm{CH}_{2}\right), 36.5\left(\mathrm{CH}_{2}\right), 21.6\left(\mathrm{CH}_{3}\right)$. HRMS (ESI) $m / z:[\mathrm{M}+\mathrm{Na}]^{+}$calcd for $\mathrm{C}_{21} \mathrm{H}_{19} \mathrm{NO}_{3} \mathrm{SNa}^{+}$388.0978; found 388.0974.

4-Methyl- $N$-(prop-2-yn-1-yl)- $N$-((5-(thiophen-2-yl)furan-2yl)methyl)benzenesulfonamide, int-8. Synthesized according to the general procedure GP2. White solid, $2.42 \mathrm{~g}$, $65 \%$ yield ( 3 steps), $\mathrm{mp}$ $127-131{ }^{\circ} \mathrm{C}$ (decomposition). $R_{f} 0.46(8 / 2 \mathrm{DCM} / \mathrm{PE}) .{ }^{1} \mathrm{H}$ NMR $(600$ $\left.\mathrm{MHz}, \mathrm{CDCl}_{3}\right) \delta(\mathrm{ppm}): 7.74(\mathrm{~d}, 2 \mathrm{H}, J=8.2 \mathrm{~Hz}), 7.27(\mathrm{~d}, 2 \mathrm{H}, J=8.3$ $\mathrm{Hz}), 7.22(\mathrm{~d}, 1 \mathrm{H}, J=5.0 \mathrm{~Hz}), 7.16(\mathrm{~d}, 1 \mathrm{H}, J=3.5 \mathrm{~Hz}), 7.03-7.00(\mathrm{~m}$, $1 \mathrm{H}), 6.38(\mathrm{~d}, 1 \mathrm{H}, J=3.3 \mathrm{~Hz}), 6.34(\mathrm{~d}, 1 \mathrm{H}, J=3.3 \mathrm{~Hz}), 4.49(\mathrm{~s}, 2 \mathrm{H})$, $4.08(\mathrm{~d}, 2 \mathrm{H}, J=2.4 \mathrm{~Hz}), 2.39(\mathrm{~s}, 3 \mathrm{H}), 2.11(\mathrm{t}, 1 \mathrm{H}, J=2.5 \mathrm{~Hz}) .{ }^{13} \mathrm{C}\left\{{ }^{1} \mathrm{H}\right\}$ NMR $\left(150 \mathrm{MHz}, \mathrm{CDCl}_{3}\right) \delta(\mathrm{ppm}): 149.9(\mathrm{Cq}), 147.9(\mathrm{Cq}), 143.8$ $(\mathrm{Cq}), 136.1(\mathrm{Cq}), 133.4(\mathrm{Cq}), 129.7(\mathrm{CH}), 127.8(\mathrm{CH}), 127.7(\mathrm{CH})$, $124.5(\mathrm{CH}), 123.0(\mathrm{CH}), 112.2(\mathrm{CH}), 105.8(\mathrm{CH}), 76.7(\mathrm{Cq}), 74.1$ $(\mathrm{CH}), 43.0\left(\mathrm{CH}_{2}\right), 36.5\left(\mathrm{CH}_{2}\right), 21.6\left(\mathrm{CH}_{3}\right)$. HRMS (ESI) $m / z:[\mathrm{M}+$ $\mathrm{Na}]^{+}$calcd for $\mathrm{C}_{19} \mathrm{H}_{17} \mathrm{NO}_{3} \mathrm{~S}_{2} \mathrm{Na}^{+}$394.0542; found 394.0528.

$N$-([2,2'-Bifuran]-5-ylmethyl)-4-methyl- $N$-(prop-2-yn-1-yl)benzenesulfonamide, int-9. Synthesized according to the general procedure GP2. White solid, $2.22 \mathrm{~g}$, 63\% yield (3 steps), mp 136-138 ${ }^{\circ} \mathrm{C}$ (EtOAc). $R_{f} 0.43$ (7/3 DCM/PE). ${ }^{1} \mathrm{H}$ NMR $\left(600 \mathrm{MHz}, \mathrm{CDCl}_{3}\right) \delta$ (ppm): 7.76-7.73 (m, 2H), 7.39 (dd, $1 \mathrm{H}, J=1.7,0.7 \mathrm{~Hz}), 7.28(\mathrm{~d}, 2 \mathrm{H}$, $J=8.0 \mathrm{~Hz}), 3.47(\mathrm{~d}, 1 \mathrm{H}, J=3.1 \mathrm{~Hz}), 6.45-6.43(\mathrm{~m}, 2 \mathrm{H}), 6.35(\mathrm{~d}, 1 \mathrm{H}, J$ $=3.4 \mathrm{~Hz}), 4.48(\mathrm{~s}, 2 \mathrm{H}), 4.06(\mathrm{~d}, 2 \mathrm{H}, J=2.4 \mathrm{~Hz}), 2.40(\mathrm{~s}, 3 \mathrm{H}), 2.09(\mathrm{t}$, $1 \mathrm{H}, J=2.5 \mathrm{~Hz}) \cdot{ }^{13} \mathrm{C}\left\{{ }^{1} \mathrm{H}\right\}$ NMR $\left(150 \mathrm{MHz}, \mathrm{CDCl}_{3}\right) \delta(\mathrm{ppm}): 147.9$ $(\mathrm{Cq}), 147.1(\mathrm{Cq}), 146.3(\mathrm{Cq}), 143.8(\mathrm{Cq}), 142.0(\mathrm{CH}), 136.0(\mathrm{Cq})$,
$129.6(\mathrm{CH}), 127.8(\mathrm{CH}), 112.0(\mathrm{CH}), 111.5(\mathrm{CH}), 105.8(\mathrm{CH}), 105.6$ $(\mathrm{CH}), 76.6(\mathrm{Cq}), 74.2(\mathrm{CH}), 43.0\left(\mathrm{CH}_{2}\right), 36.4\left(\mathrm{CH}_{2}\right), 21.7\left(\mathrm{CH}_{3}\right)$. HRMS (ESI) $m / z$ : $[\mathrm{M}+\mathrm{Na}]^{+}$calcd for $\mathrm{C}_{19} \mathrm{H}_{17} \mathrm{NO}_{4} \mathrm{SNa}^{+}$378.0770; found 378.0781.

General Procedure A for the Synthesis of Terminal Alkynes with $O$ Linker (GP3A). ${ }^{31}$ To a $1.0 \mathrm{M}$ solution of furaldehyde (1.0 equiv, $10 \mathrm{mmol}$ ) in $\mathrm{MeOH}, \mathrm{NaBH}_{4}$ (1.0 equiv) was added portionwise at $0{ }^{\circ} \mathrm{C}$. The mixture was allowed to reach room temperature and stirred overnight. Then, $\mathrm{MeOH}$ was evaporated to half of the initial volume under reduced pressure. Water was added, and the mixture was extracted three times with DCM; the combined organic layers were dried over $\mathrm{Na}_{2} \mathrm{SO}_{4}$ and filtered, and the volatiles were evaporated under reduced pressure to obtain the crude furyl alcohol, which was used for the next step without further purification.

To a 1.0 M solution of the crude furanyl alcohol in anhydrous DMF, at $0{ }^{\circ} \mathrm{C}$ under a $\mathrm{N}_{2}$ atmosphere, $\mathrm{NaH}$ (1.5 equiv) was added portionwise. The mixture was stirred at $0{ }^{\circ} \mathrm{C}$ for $15 \mathrm{~min}$, then propargyl bromide (1.5 equiv) was added, and the mixture was allowed to reach room temperature and stirred overnight. Then, the mixture was cooled down to $0{ }^{\circ} \mathrm{C}$, water was added, and the mixture was extracted three times with DCM; the combined organic layers were washed three times with water, dried over anhydrous $\mathrm{Na}_{2} \mathrm{SO}_{4}$, and filtered, and the volatiles were removed under reduced pressure. The crude product was purified by flash column chromatography to obtain the pure O-propargyl product.

General Procedure B for the Synthesis of Terminal Alkynes with $O$ Linker (GP3B). ${ }^{32}$ To a $0.5 \mathrm{M}$ solution of furaldehyde (1.0 equiv, $10 \mathrm{mmol}$ ) in anhydrous $\mathrm{THF}$, at $0{ }^{\circ} \mathrm{C}$ under a $\mathrm{N}_{2}$ atmosphere, a solution of Grignard reagent (1.2 equiv) was added. The mixture was allowed to reach room temperature and stirred for $1-2 \mathrm{~h}$ (GC-MS monitoring). Then, a saturated $\mathrm{NH}_{4} \mathrm{Cl}$ solution was added, and the mixture was extracted three times with $\mathrm{DCM}$; the combined organic layers were dried over $\mathrm{Na}_{2} \mathrm{SO}_{4}$, filtered and the volatiles were evaporated under reduced pressure to obtain the crude furyl alcohol, which was used for the next step without further purification.

To a $1.0 \mathrm{M}$ solution of the crude furanyl alcohol in anhydrous DMF, at $0{ }^{\circ} \mathrm{C}$ under a $\mathrm{N}_{2}$ atmosphere, $\mathrm{NaH}$ (1.5 equiv) was added portionwise. The mixture was stirred at $0{ }^{\circ} \mathrm{C}$ for $15 \mathrm{~min}$, then propargyl bromide (1.5 equiv) was added, and the mixture was allowed to reach room temperature and stirred overnight. Then, the mixture was cooled down to $0{ }^{\circ} \mathrm{C}$, water was added, and the mixture was extracted three times with DCM; the combined organic layers were washed three times with water, dried over anhydrous $\mathrm{Na}_{2} \mathrm{SO}_{4}$, and filtered, and the volatiles were removed under reduced pressure. The crude product was purified by flash column chromatography to obtain the pure O-propargyl product.

2-Methyl-5-((prop-2-yn-1-yloxy)methyl)furan, ${ }^{31}$ int-10. Synthesized according to the general procedure GP3A. Pale yellow oil, 1.01 g, 67\% yield (2 steps). $R_{f} 0.18$ (99/1 PE/EtOAc). ${ }^{1} \mathrm{H}$ NMR $(600 \mathrm{MHz}$, $\left.\mathrm{CDCl}_{3}\right) \delta(\mathrm{ppm}): 6.25(\mathrm{dd}, 1 \mathrm{H}, J=3.0,0.6 \mathrm{~Hz}), 5.92(\mathrm{dq}, 1 \mathrm{H}, J=3.1$ $\mathrm{Hz}, 1.1 \mathrm{~Hz}), 4.50(\mathrm{~s}, 2 \mathrm{H}), 4.16(\mathrm{~d}, 2 \mathrm{H}, J=2.4 \mathrm{~Hz}), 2.43(\mathrm{~s}, 3 \mathrm{H}), 2.29(\mathrm{~d}$ $3 \mathrm{H}, J=1.0 \mathrm{~Hz}$ ). GC-MS $m / z(\%): 150[\mathrm{M}]^{+}(30), 110(21), 95$ (100).

2-Methyl-5-(1-(prop-2-yn-1-yloxy)propyl)furan, ${ }^{32}$ int-11. Synthesized according to the general procedure GP3B with EtMgBr

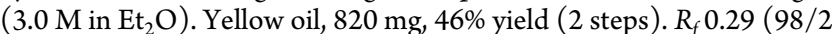
PE/EtOAc). ${ }^{1} \mathrm{H}$ NMR $\left(600 \mathrm{MHz}, \mathrm{CDCl}_{3}\right) \delta(\mathrm{ppm}): 6.17(\mathrm{~d}, 1 \mathrm{H}, J=$ $3.0 \mathrm{~Hz}), 5.89(\mathrm{dq}, 1 \mathrm{H}, J=3.1,1.0 \mathrm{~Hz}), 4.34(\mathrm{t}, 1 \mathrm{H}, J=7.1 \mathrm{~Hz}), 4.13(\mathrm{dd}$, $1 \mathrm{H}, J=15.8,2.4 \mathrm{~Hz}), 3.95(\mathrm{dd}, 1 \mathrm{H}, J=15.8,2.4 \mathrm{~Hz}), 2.38(\mathrm{t}, 1 \mathrm{H}, J=2.4$ $\mathrm{Hz}), 2.27(\mathrm{~d}, 3 \mathrm{H}, J=1.0 \mathrm{~Hz}), 1.95-1.81(\mathrm{~m}, 2 \mathrm{H}), 0.90(\mathrm{t}, 3 \mathrm{H}, J=7.5$ Hz). GC-MS $m / z(\%): 178[\mathrm{M}]^{+}(5), 149$ (100), 123 (38), 120 (24), 109 (36), 91 (22), 77 (33), 43 (41).

2-(2,2-Dimethyl-1-(prop-2-yn-1-yloxy)propyl)-5-methylfuran, int-12. Synthesized according to the general procedure GP3B with $t$ - BuMgCl $(1.0 \mathrm{M}$ in THF). Yellow oil, $720 \mathrm{mg}$, 35\% yield (2 steps). $R_{f} 0.45$ (99/1 PE/EtOAc). ${ }^{1} \mathrm{H} \mathrm{NMR}\left(600 \mathrm{MHz}, \mathrm{CDCl}_{3}\right) \delta$ $(\mathrm{ppm}): 6.13(\mathrm{~d}, 1 \mathrm{H}, J=3.0 \mathrm{~Hz}), 5.91(\mathrm{dd}, 1 \mathrm{H}, J=3.0,1.0 \mathrm{~Hz}), 4.16(\mathrm{dd}$, $1 \mathrm{H}, J=15.6,2.4 \mathrm{~Hz}), 4.12(\mathrm{~s}, 1 \mathrm{H}), 3.87(\mathrm{dd}, 1 \mathrm{H}, J=16.0,2.3 \mathrm{~Hz}), 2.37$ $(\mathrm{t}, 1 \mathrm{H}, J=2.4 \mathrm{~Hz}), 2.28(\mathrm{~d}, 3 \mathrm{H}, J=0.8 \mathrm{~Hz}), 0.96(\mathrm{~s}, 9 \mathrm{H}) .{ }^{13} \mathrm{C}\left\{{ }^{1} \mathrm{H}\right\}$ NMR $\left(150 \mathrm{MHz}, \mathrm{CDCl}_{3}\right) \delta(\mathrm{ppm}): 151.8(\mathrm{Cq}), 151.0$ (Cq), 110.4 $(\mathrm{CH}), 105.9(\mathrm{CH}), 82.1(\mathrm{CH}), 80.3(\mathrm{Cq}), 74.0(\mathrm{CH}), 56.0\left(\mathrm{CH}_{2}\right)$, 
$35.3(\mathrm{Cq}), 26.4\left(\mathrm{CH}_{3}\right), 13.8\left(\mathrm{CH}_{3}\right)$. HRMS (ESI) $m / z:[\mathrm{M}+\mathrm{Na}]^{+}$ calcd for $\mathrm{C}_{13} \mathrm{H}_{18} \mathrm{O}_{2} \mathrm{Na}^{+} 229.1199$; found 229.1193 .

General Procedure for the Sonogashira Coupling (GP4). ${ }^{33}$ In a vial under a $\mathrm{N}_{2}$ atmosphere, a $2.0 \mathrm{M}$ solution of the terminal alkyne (1.0 equiv, $1.0-2.0 \mathrm{mmol}$ ) in anhydrous THF was prepared. The aryl iodide or aryl bromide (1.5 equiv) and $\mathrm{Et}_{3} \mathrm{~N}$ (2.0 equiv) were added, and the mixture was degassed for $10-15 \mathrm{~min}$. Then, $\left[\left(\mathrm{Ph}_{3} \mathrm{P}\right)_{2} \mathrm{PdCl}_{2}\right]$ ( 0.02 equiv) and $\mathrm{CuI}$ ( 0.04 equiv) were added, and the mixture was stirred at room temperature (for ArI) or at $60{ }^{\circ} \mathrm{C}$ (for $\mathrm{ArBr}$ ) overnight. After that time, a saturated $\mathrm{NH}_{4} \mathrm{Cl}$ solution was added, and the mixture was extracted three times with EtOAc; the combined organic layers were dried over $\mathrm{Na}_{2} \mathrm{SO}_{4}$ and filtered, and the volatiles were removed under reduced pressure. The crude product was purified by flash column chromatography to obtain pure aryl alkynes $\mathbf{1}$ as the product.

4-Methyl- $N$-((5-methylfuran-2-yl)methyl)- $N$-(3-phenylprop2-yn-1-yl)benzenesulfonamide, 1a. Synthesized according to the general procedure GP4 at rt with PhI. White solid, $252 \mathrm{mg}, 68 \%$ yield, mp $81-83{ }^{\circ} \mathrm{C}\left(\mathrm{Et}_{2} \mathrm{O}\right) \cdot R_{f} 0.17$ (95/5 PE/EtOAc). ${ }^{1} \mathrm{H} \mathrm{NMR}(600 \mathrm{MHz}$, $\left.\mathrm{CDCl}_{3}\right) \delta(\mathrm{ppm}): 7.78(\mathrm{~d}, 2 \mathrm{H}, J=8.2 \mathrm{~Hz}), 7.31-7.23(\mathrm{~m}, 5 \mathrm{H}), 7.09(\mathrm{~d}$, $2 \mathrm{H}, J=8.1 \mathrm{~Hz}), 6.20(\mathrm{~d}, 1 \mathrm{H}, J=2.8 \mathrm{~Hz}), 5.88(\mathrm{~d}, 1 \mathrm{H}, J=2.0 \mathrm{~Hz}), 4.42$ $(\mathrm{s}, 2 \mathrm{H}), 4.24(\mathrm{~s}, 2 \mathrm{H}), 2.34(\mathrm{~s}, 3 \mathrm{H}), 2.22(\mathrm{~s}, 3 \mathrm{H}) .{ }^{13} \mathrm{C}\left\{{ }^{1} \mathrm{H}\right\}$ NMR $(150$ $\left.\mathrm{MHz}, \mathrm{CDCl}_{3}\right) \delta(\mathrm{ppm}): 153.0(\mathrm{Cq}), 146.7(\mathrm{Cq}), 143.6(\mathrm{Cq}), 136.2$ $(\mathrm{Cq}), 131.7(\mathrm{CH}), 129.6(\mathrm{CH}), 128.5(\mathrm{CH}), 128.3(\mathrm{CH}), 128.0(\mathrm{CH})$, $122.4(\mathrm{Cq}), 111.2(\mathrm{CH}), 106.4(\mathrm{CH}), 85.9(\mathrm{Cq}), 81.9(\mathrm{Cq}), 43.4$ $\left(\mathrm{CH}_{2}\right), 37.1\left(\mathrm{CH}_{2}\right), 21.6\left(\mathrm{CH}_{3}\right), 13.7\left(\mathrm{CH}_{3}\right)$. HRMS (ESI) $\mathrm{m} / z:[\mathrm{M}+$ $\mathrm{Na}]^{+}$calcd for $\mathrm{C}_{22} \mathrm{H}_{21} \mathrm{NO}_{3} \mathrm{SNa}^{+} 402.1134$; found 402.1127 .

$\mathrm{N}$-(3-(4-Methoxyphenyl)prop-2-yn-1-yl)-4-methyl-N-((5methylfuran-2-yl)methyl)benzenesulfonamide, $1 \mathrm{~b}$. Synthesized according to the general procedure GP4 at rt with $p$-iodoanisole. White solid, $568 \mathrm{mg}$, $69 \%$ yield, $\mathrm{mp} 92-94{ }^{\circ} \mathrm{C}\left(\mathrm{Et}_{2} \mathrm{O}\right) \cdot R_{f} 0.28$ (9/1 PE/ EtOAc). ${ }^{1} \mathrm{H}$ NMR $\left(600 \mathrm{MHz}, \mathrm{CDCl}_{3}\right) \delta(\mathrm{ppm}): 7.79-7.77(\mathrm{~m}, 2 \mathrm{H})$, $7.27-7.25(\mathrm{~m}, 2 \mathrm{H}), 7.06-7.03(\mathrm{~m}, 2 \mathrm{H}), 6.79-6.76(\mathrm{~m}, 2 \mathrm{H}), 6.19$ (d, $1 \mathrm{H}, J=3.0 \mathrm{~Hz}), 5.87(\mathrm{dq}, 1 \mathrm{H}, J=3.0,1.0 \mathrm{~Hz}), 4.41(\mathrm{~s}, 2 \mathrm{H9}, 4.22(\mathrm{~s}$, $2 \mathrm{H}), 3.80(\mathrm{~s}, 3 \mathrm{H}), 2.36(\mathrm{~s}, 3 \mathrm{H}), 2.22(\mathrm{~d}, 3 \mathrm{H}, J=0.9 \mathrm{~Hz}) .{ }^{13} \mathrm{C}\left\{{ }^{1} \mathrm{H}\right\} \mathrm{NMR}$

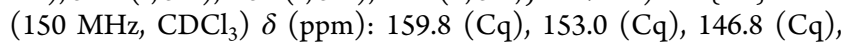
$143.5(\mathrm{Cq}), 136.3(\mathrm{Cq}), 133.2(\mathrm{CH}), 129.6(\mathrm{CH}), 128.0(\mathrm{CH}), 114.5$ (Cq), $113.9(\mathrm{CH}), 111.1(\mathrm{CH}), 106.4(\mathrm{CH}), 85.8(\mathrm{Cq}), 80.4(\mathrm{Cq})$, 55.4 $\left(\mathrm{CH}_{3}\right), 43.3\left(\mathrm{CH}_{2}\right), 37.2\left(\mathrm{CH}_{2}\right), 21.6\left(\mathrm{CH}_{3}\right), 13.7\left(\mathrm{CH}_{3}\right)$. HRMS (ESI) $\mathrm{m} / z$ : $[\mathrm{M}+\mathrm{Na}]^{+}$calcd for $\mathrm{C}_{23} \mathrm{H}_{23} \mathrm{NO}_{4} \mathrm{SNa}^{+} 432.1240$; found 432.1230 .

$\mathrm{N}$-(3-(4-Hydroxyphenyl)prop-2-yn-1-yl)-4-methyl-N-((5methylfuran-2-yl)methyl)benzenesulfonamide, 1c. Synthesized according to the general procedure GP4 at rt with $p$-iodophenol. White solid, $236 \mathrm{mg}, 60 \%$ yield, $\mathrm{mp} 100-103{ }^{\circ} \mathrm{C}\left(\mathrm{DCM} /\right.$ pentane). $R_{f} 0.27$ (8/ $2 \mathrm{PE} / \mathrm{EtOAc}) .{ }^{1} \mathrm{H}$ NMR $\left(600 \mathrm{MHz}, \mathrm{CDCl}_{3}\right) \delta(\mathrm{ppm}): 7.79(\mathrm{~m}, 2 \mathrm{H})$, 7.27-7.24 (m, 2H), 7.01-6.98 (m, 2H), 6.73-6.70 (m, 2H), 6.19 (d, $1 \mathrm{H}, J=3.1 \mathrm{~Hz}), 5.87(\mathrm{dd}, 1 \mathrm{H}, J=3.0,1.0 \mathrm{~Hz}), 5.03(\mathrm{br} \mathrm{s}, 1 \mathrm{H}), 4.41(\mathrm{~s}$, $2 \mathrm{H}), 4.21(\mathrm{~s}, 3 \mathrm{H}), 2.36(\mathrm{~s}, 3 \mathrm{H}), 2.22(\mathrm{~s}, 3 \mathrm{H}) .{ }^{13} \mathrm{C}\left\{{ }^{1} \mathrm{H}\right\}$ NMR $(150$ $\left.\mathrm{MHz}, \mathrm{CDCl}_{3}\right) \delta(\mathrm{ppm}): 156.1(\mathrm{Cq}), 153.0(\mathrm{Cq}), 146.7(\mathrm{Cq}), 143.7$ $(\mathrm{Cq}), 136.1(\mathrm{Cq}), 133.3(\mathrm{CH}), 129.6(\mathrm{CH}), 128.0(\mathrm{CH}), 115.4(\mathrm{CH})$, $114.5(\mathrm{Cq}), 111.2(\mathrm{CH}), 106.4(\mathrm{CH}), 85.9(\mathrm{Cq}), 80.2(\mathrm{Cq}), 43.2$ $\left(\mathrm{CH}_{2}\right), 37.2\left(\mathrm{CH}_{2}\right), 21.6\left(\mathrm{CH}_{3}\right), 13.7\left(\mathrm{CH}_{3}\right)$. HRMS (ESI) $\mathrm{m} / z$ : $[\mathrm{M}+$ $\mathrm{H}]^{+}$calcd for $\mathrm{C}_{22} \mathrm{H}_{22} \mathrm{NO}_{4} \mathrm{~S}^{+} 396.1264$; found 396.1265.

4-Methyl- $N$-((5-methylfuran-2-yl)methyl)- $N$-(3-(p-tolyl)prop-2-yn-1-yl)benzenesulfonamide, 1d. Synthesized according to the general procedure GP5 at rt with $p$-iodotoluene. White solid, 272 mg, $69 \%$ yield, mp $58-60{ }^{\circ} \mathrm{C}\left(\mathrm{Et}_{2} \mathrm{O}\right) . R_{f} 0.18$ (93/7 PE/EtOAc). ${ }^{1} \mathrm{H}$ NMR $\left(600 \mathrm{MHz}, \mathrm{CDCl}_{3}\right) \delta(\mathrm{ppm}): 7.79-7.77(\mathrm{~m}, 2 \mathrm{H}), 7.26(\mathrm{~d}, 2 \mathrm{H}, J$ $=7.9 \mathrm{~Hz}), 7.05(\mathrm{~d}, 2 \mathrm{H}, J=7.8 \mathrm{~Hz}), 6.99-6.97(\mathrm{~m}, 2 \mathrm{H}), 6.19(\mathrm{~d}, 1 \mathrm{H}, J=$ $3.0 \mathrm{~Hz}), 5.88-5.87(\mathrm{~m}, 1 \mathrm{H}), 4.41(\mathrm{~s}, 2 \mathrm{H}), 4.22(\mathrm{~s}, 2 \mathrm{H}), 2.36(\mathrm{~s}, 3 \mathrm{H})$, $2.33(\mathrm{~s}, 3 \mathrm{H}), 2.22(\mathrm{~d}, 3 \mathrm{H}, J=1.0 \mathrm{~Hz}) .{ }^{13} \mathrm{C}\left\{{ }^{1} \mathrm{H}\right\}$ NMR $(150 \mathrm{MHz}$, $\left.\mathrm{CDCl}_{3}\right) \delta(\mathrm{ppm}): 153.0(\mathrm{Cq}), 146.7(\mathrm{Cq}), 143.6(\mathrm{Cq}), 138.7(\mathrm{Cq})$, $136.2(\mathrm{Cq}), 131.6(\mathrm{CH}), 129.6(\mathrm{CH}), 129.0(\mathrm{CH}), 128.0(\mathrm{CH}), 119.3$ $(\mathrm{Cq}), 111.1(\mathrm{CH}), 106.4(\mathrm{CH}), 86.0(\mathrm{Cq}), 81.1(\mathrm{Cq}), 43.3\left(\mathrm{CH}_{2}\right)$, $37.1\left(\mathrm{CH}_{2}\right), 21.6\left(2 \times \mathrm{CH}_{3}\right), 13.7\left(\mathrm{CH}_{3}\right)$. HRMS (ESI) $m / z:[\mathrm{M}+\mathrm{Na}]^{+}$ calcd for $\mathrm{C}_{23} \mathrm{H}_{23} \mathrm{NO}_{3} \mathrm{SNa}^{+} 416.1291$; found 416.1281.

Methyl 4-(3-((4-Methyl-N-((5-methylfuran-2-yl)methyl)phenyl)sulfonamido)prop-1-yn-1-yl)benzoate, 1e. Synthesized according to the general procedure GP4 at rt with methyl $p$ iodobenzoate. White solid, $373 \mathrm{mg}, 85 \%$ yield, mp $133-135{ }^{\circ} \mathrm{C}$
$\left(\mathrm{Et}_{2} \mathrm{O}\right) . R_{\mathrm{f}} 0.26$ (9/1 PE/EtOAc). ${ }^{1} \mathrm{H}$ NMR $\left(600 \mathrm{MHz}, \mathrm{CDCl}_{3}\right) \delta$ (ppm): 7.93-7.90 (m, 2H), 7.79-7.76 (m, 2H), 7.26-7.24 (m, 2H), $7.15-7.13(\mathrm{~m}, 2 \mathrm{H}), 6.19(\mathrm{~d}, 1 \mathrm{H}, J=3.1 \mathrm{~Hz}), 5.89-5.87(\mathrm{dq}, 1 \mathrm{H}, J=$ $3.1,1.2 \mathrm{~Hz}), 4.42(\mathrm{~s}, 2 \mathrm{H}), 4.25(\mathrm{~s}, 2 \mathrm{H}), 3.92(\mathrm{~d}, 3 \mathrm{H}, J=0.7 \mathrm{~Hz}), 2.34(\mathrm{~s}$, $3 \mathrm{H}), 2.21(\mathrm{~s}, 3 \mathrm{H}) .{ }^{13} \mathrm{C}\left\{{ }^{1} \mathrm{H}\right\} \mathrm{NMR}\left(150 \mathrm{MHz}, \mathrm{CDCl}_{3}\right) \delta(\mathrm{ppm}): 166.5$ $(\mathrm{Cq}), 153.1(\mathrm{Cq}), 146.6(\mathrm{Cq}), 143.7(\mathrm{Cq}), 136.1(\mathrm{Cq}), 131.6(\mathrm{CH})$, $129.8(\mathrm{Cq}), 129.7(\mathrm{CH}), 129.4(\mathrm{CH}), 128.0(\mathrm{CH}), 127.0(\mathrm{Cq}), 111.2$ $(\mathrm{CH}), 106.5(\mathrm{CH}), 85.1(\mathrm{Cq}), 85.1(\mathrm{Cq}), 52.4\left(\mathrm{CH}_{3}\right), 43.6\left(\mathrm{CH}_{2}\right)$, $37.1\left(\mathrm{CH}_{2}\right), 21.6\left(\mathrm{CH}_{3}\right), 13.7\left(\mathrm{CH}_{3}\right)$. HRMS (ESI) $\mathrm{m} / z:[\mathrm{M}+\mathrm{Na}]^{+}$ calcd for $\mathrm{C}_{24} \mathrm{H}_{23} \mathrm{NO}_{5} \mathrm{SNa}^{+} 460.1189$; found 460.1180 .

$\mathrm{N}$-(3-(4-Acetylphenyl)prop-2-yn-1-yl)-4-methyl- $N$-((5-methylfuran-2-yl)methyl)benzenesulfonamide, 1f. Synthesized according to the general procedure GP4 at rt with $4^{\prime}$-iodoacetophenone. White solid, $307 \mathrm{mg}$, $73 \%$ yield, $\mathrm{mp} 91-93{ }^{\circ} \mathrm{C}\left(\mathrm{Et}_{2} \mathrm{O}\right) \cdot R_{f} 0.32(8 / 2 \mathrm{PE} /$ EtOAc). ${ }^{1} \mathrm{H}$ NMR $\left(600 \mathrm{MHz}, \mathrm{CDCl}_{3}\right) \delta(\mathrm{ppm}): 7.85-7.83(\mathrm{~m}, 2 \mathrm{H})$, 7.79-7.77 (m, 2H), 7.28-7.25 (m, 2H), 7.19-7.16 (m, 2H), $6.18(\mathrm{~d}$, $1 \mathrm{H}, J=3.0 \mathrm{~Hz}), 5.88(\mathrm{dq}, 1 \mathrm{H}, J=3.0,1.0 \mathrm{~Hz}), 4.42(\mathrm{~s}, 2 \mathrm{H}), 4.26(\mathrm{~s}$, $2 \mathrm{H}), 2.59(\mathrm{~s}, 3 \mathrm{H}), 2.35$ (s, 3H), $2.21(\mathrm{~s}, 3 \mathrm{H}) .{ }^{13} \mathrm{C}\left\{{ }^{1} \mathrm{H}\right\}$ NMR $(150$ $\left.\mathrm{MHz}_{\mathrm{CDCl}}\right) \delta(\mathrm{ppm}): 197.3(\mathrm{Cq}), 153.1(\mathrm{Cq}), 146.6(\mathrm{Cq}), 143.7$ $(\mathrm{Cq}), 136.5(\mathrm{Cq}), 136.2(\mathrm{Cq}), 131.8(\mathrm{CH}), 129.7(\mathrm{CH}), 128.2(\mathrm{CH})$, $128.0(\mathrm{CH}), 127.2(\mathrm{Cq}), 111.2(\mathrm{CH}), 106.5(\mathrm{CH}), 85.6(\mathrm{Cq}), 85.0$ (Cq), $43.6\left(\mathrm{CH}_{2}\right)$, 37.1 $\left(\mathrm{CH}_{2}\right), 26.7\left(\mathrm{CH}_{3}\right), 21.6\left(\mathrm{CH}_{3}\right), 13.7\left(\mathrm{CH}_{3}\right)$. HRMS (ESI) $\mathrm{m} / z$ : $[\mathrm{M}+\mathrm{H}]^{+}$calcd for $\mathrm{C}_{24} \mathrm{H}_{24} \mathrm{NO}_{4} \mathrm{~S}^{+} 422.1421$; found 422.1416.

$\mathrm{N}$-(3-(4-fluorophenyl)prop-2-yn-1-yl)-4-methyl- $\mathrm{N}$-((5-methylfuran-2-yl)methyl)benzenesulfonamide, 1g. Synthesized according to the general procedure GP4 at rt with $p$-fluoroiodobenzene. White solid, $339 \mathrm{mg}$, $86 \%$ yield, $\mathrm{mp} 86-88^{\circ} \mathrm{C}\left(\mathrm{Et}_{2} \mathrm{O}\right) \cdot R_{f} 0.36(9 / 1 \mathrm{PE} /$ EtOAc). ${ }^{1} \mathrm{H}$ NMR $\left(600 \mathrm{MHz}, \mathrm{CDCl}_{3}\right) \delta(\mathrm{ppm}): 7.78(\mathrm{~d}, 2 \mathrm{H}, J=8.3$ $\mathrm{Hz}), 7.27-7.25(\mathrm{~m}, 2 \mathrm{H}), 7.10-7.06(\mathrm{~m}, 2 \mathrm{H}), 6.97-6.92(\mathrm{~m}, 2 \mathrm{H}), 6.18$ $(\mathrm{d}, 1 \mathrm{H}, J=3.0 \mathrm{~Hz}), 5.88(\mathrm{~d}, 1 \mathrm{H}, J=2.9 \mathrm{~Hz}), 4.41(\mathrm{~s}, 2 \mathrm{H}), 4.22(\mathrm{~s}, 2 \mathrm{H})$, $2.36(\mathrm{~s}, 3 \mathrm{H}), 2.21(\mathrm{~s}, 3 \mathrm{H}) .{ }^{13} \mathrm{C}\left\{{ }^{1} \mathrm{H}\right\} \operatorname{NMR}\left(150 \mathrm{MHz}, \mathrm{CDCl}_{3}\right) \delta(\mathrm{ppm})$ : $162.6(\mathrm{Cq}, \mathrm{d}, J=250.0 \mathrm{~Hz}), 153.0(\mathrm{Cq}), 146.7(\mathrm{Cq}), 143.6(\mathrm{Cq}), 136.2$ $(\mathrm{Cq}), 133.6(\mathrm{CH}, \mathrm{d}, J=8.6 \mathrm{~Hz}), 129.6(\mathrm{CH}), 128.0(\mathrm{CH}), 118.5(\mathrm{Cq}$, d, $J=3.3 \mathrm{~Hz}), 115.6(\mathrm{CH}, \mathrm{d}, J=21.6 \mathrm{~Hz}), 111.1(\mathrm{CH}), 106.4(\mathrm{CH})$, $84.8(\mathrm{Cq}), 81.7(\mathrm{Cq}), 43.4\left(\mathrm{CH}_{2}\right), 37.1\left(\mathrm{CH}_{2}\right), 21.6\left(\mathrm{CH}_{3}\right), 13.7$ $\left(\mathrm{CH}_{3}\right) \cdot{ }^{19} \mathrm{~F}$ NMR $\left(564 \mathrm{MHz}, \mathrm{CDCl}_{3}\right) \delta(\mathrm{ppm}):-110.4(\mathrm{tt}, J=8.6,5.4$ $\mathrm{Hz}$ ). HRMS (ESI) $m / z:[\mathrm{M}+\mathrm{Na}]^{+}$calcd for $\mathrm{C}_{22} \mathrm{H}_{20} \mathrm{FNO}_{3} \mathrm{SNa}^{+}$ 420.1040; found 420.1027 .

4-methyl- $\mathrm{N}$-((5-methylfuran-2-yl)methyl)- $\mathrm{N}$-(3-(thiophen-2yl)prop-2-yn-1-yl)benzenesulfonamide, 1h. Synthesized according to the general procedure GP4 at $60{ }^{\circ} \mathrm{C}$ with 2-bromothiophene. White solid, $166 \mathrm{mg}$, $43 \%$ yield, $\mathrm{mp} 87-89^{\circ} \mathrm{C}\left(\mathrm{Et}_{2} \mathrm{O}\right) . R_{f} 0.34(9 / 1 \mathrm{PE} /$ EtOAc). ${ }^{1} \mathrm{H}$ NMR $\left(600 \mathrm{MHz}, \mathrm{CDCl}_{3}\right) \delta(\mathrm{ppm}): 7.79-7.76(\mathrm{~m}, 2 \mathrm{H})$, $7.30-7.27(\mathrm{~m}, 2 \mathrm{H}), 7.21(\mathrm{dd}, 1 \mathrm{H}, J=5.1,1.2 \mathrm{~Hz}), 6.25(\mathrm{dd}, 1 \mathrm{H}, J=3.6$, $1.2 \mathrm{~Hz}), 6.92(\mathrm{dd}, 1 \mathrm{H}, J=5.2,3.6 \mathrm{~Hz}), 6.19(\mathrm{~d}, 1 \mathrm{H}, J=3.0 \mathrm{~Hz}), 5.88$ $(\mathrm{dq}, 1 \mathrm{H}, J=3.0,1.0 \mathrm{~Hz}), 4.39(\mathrm{~s}, 2 \mathrm{H}), 4.25(\mathrm{~s}, 2 \mathrm{H}), 2.38(\mathrm{~s}, 3 \mathrm{H}), 2.22$ $(\mathrm{d}, 3 \mathrm{H}, J=1.1 \mathrm{~Hz}) .{ }^{13} \mathrm{C}\left\{{ }^{1} \mathrm{H}\right\} \mathrm{NMR}\left(150 \mathrm{MHz}, \mathrm{CDCl}_{3}\right) \delta(\mathrm{ppm}): 153.1$ $(\mathrm{Cq}), 146.6(\mathrm{Cq}), 143.7(\mathrm{Cq}), 136.0(\mathrm{Cq}), 132.4(\mathrm{CH}), 129.7(\mathrm{CH})$, $127.9(\mathrm{CH}), 127.4(\mathrm{CH}), 126.9(\mathrm{CH}), 122.3(\mathrm{Cq}), 111.2(\mathrm{CH}), 106.5$ $(\mathrm{CH}), 85.9(\mathrm{Cq}), 79.1(\mathrm{Cq}), 43.5\left(\mathrm{CH}_{2}\right), 37.3\left(\mathrm{CH}_{2}\right), 21.7\left(\mathrm{CH}_{3}\right)$, $13.7\left(\mathrm{CH}_{3}\right)$. HRMS (ESI) $\mathrm{m} / z$ : $[\mathrm{M}+\mathrm{H}]^{+}$calcd for $\mathrm{C}_{20} \mathrm{H}_{20} \mathrm{NO}_{3} \mathrm{~S}_{2}{ }^{+}$ 386.0879; found 386.0879 .

4-Methyl- $N$-((5-methylfuran-2-yl)methyl)- $N$-(3-(naphthalen1-yl)prop-2-yn-1-yl)benzenesulfonamide, 1i. Synthesized according to the general procedure GP4 at rt with 1-iodonaphthalene. White solid, $324 \mathrm{mg}, 75 \%$ yield, $\mathrm{mp} 107-109^{\circ} \mathrm{C}\left(\mathrm{Et}_{2} \mathrm{O}\right) . R_{f} 0.41(9 / 1$ $\mathrm{PE} / \mathrm{EtOAc}) .{ }^{1} \mathrm{H}$ NMR $\left(600 \mathrm{MHz}, \mathrm{CDCl}_{3}\right) \delta(\mathrm{ppm}): 7.90(\mathrm{~d}, 1 \mathrm{H}, J=$ $7.7 \mathrm{~Hz}), 7.84-7.79(\mathrm{~m}, 4 \mathrm{H}), 7.53-7.47(\mathrm{~m}, 2 \mathrm{H}), 7.17(\mathrm{~d}, 2 \mathrm{H}, J=8.0$ $\mathrm{Hz}), 6.25(\mathrm{~d}, 1 \mathrm{H}, J=3.0 \mathrm{~Hz}), 5.91-5.89(\mathrm{~m}, 1 \mathrm{H}), 4.52(\mathrm{~s}, 2 \mathrm{H}), 4.41(\mathrm{~s}$, $2 \mathrm{H}), 2.24(\mathrm{~s}, 3 \mathrm{H}), 2.17(\mathrm{~s}, 3 \mathrm{H}) .{ }^{13} \mathrm{C}\left\{{ }^{1} \mathrm{H}\right\} \mathrm{NMR}\left(150 \mathrm{MHz}, \mathrm{CDCl}_{3}\right) \delta$ $(\mathrm{ppm}): 153.1(\mathrm{Cq}), 146.7(\mathrm{Cq}), 143.7(\mathrm{Cq}), 136.1(\mathrm{Cq}), 133.2(\mathrm{Cq})$, $130.7(\mathrm{CH}), 129.7(\mathrm{CH}), 129.0(\mathrm{CH}), 128.4(\mathrm{CH}), 128.1(\mathrm{Cq}), 127.9$ $(\mathrm{CH}), 126.9(\mathrm{CH}), 126.5(\mathrm{CH}), 126.1(\mathrm{CH}), 125.1(\mathrm{CH}), 120.1(\mathrm{Cq})$, $11.2(\mathrm{CH}), 106.5(\mathrm{CH}), 86.8(\mathrm{Cq}), 84.2(\mathrm{Cq}), 43.5\left(\mathrm{CH}_{2}\right), 37.3$ $\left(\mathrm{CH}_{2}\right), 21.4\left(\mathrm{CH}_{3}\right), 13.8\left(\mathrm{CH}_{3}\right)$. HRMS (ESI) $\mathrm{m} / z:[\mathrm{M}+\mathrm{Na}]^{+}$calcd for $\mathrm{C}_{26} \mathrm{H}_{23} \mathrm{NO}_{3} \mathrm{SNa}^{+}$452.1291; found 452.1278 .

4-Methyl-N-((5-phenylfuran-2-yl)methyl)- $N$-(3-phenylprop2-yn-1-yl)benzenesulfonamide, $1 \mathbf{j}$. Synthesized according to the general procedure GP4 at rt with PhI. White solid, $357 \mathrm{mg}$, $81 \%$ yield, 
$110-111^{\circ} \mathrm{C}\left(\mathrm{CHCl}_{3}\right) \cdot R_{f} 0.41(9 / 1 \mathrm{PE} / \mathrm{EtOAc}) .{ }^{1} \mathrm{H} \mathrm{NMR}(600 \mathrm{MHz}$, $\left.\mathrm{CDCl}_{3}\right) \delta(\mathrm{ppm}): 7.80(\mathrm{~d}, 2 \mathrm{H}, J=8.3 \mathrm{~Hz}), 7.60-7.58(\mathrm{~m}, 2 \mathrm{H}), 7.35(\mathrm{t}$, $2 \mathrm{H}, J=7.7 \mathrm{~Hz}), 7.31-7.29(\mathrm{~m}, 1 \mathrm{H}), 7.26-7.22(\mathrm{~m}, 5 \mathrm{H}), 7.12(\mathrm{dd}, 2 \mathrm{H}$, $J=8.3,1.2 \mathrm{~Hz}), 6.56(\mathrm{~d}, 1 \mathrm{H}, J=3.3 \mathrm{~Hz}), 6.41(\mathrm{~d}, 1 \mathrm{H}, J=3.3 \mathrm{~Hz}), 4.56$ (s, 2H), $4.31(\mathrm{~s}, 2 \mathrm{H}), 2.32(\mathrm{~s}, 3 \mathrm{H}) \cdot{ }^{13} \mathrm{C}\left\{{ }^{1} \mathrm{H}\right\} \mathrm{NMR}\left(150 \mathrm{MHz}, \mathrm{CDCl}_{3}\right)$ $\delta(\mathrm{ppm}): 154.5(\mathrm{Cq}), 148.3(\mathrm{Cq}), 143.7(\mathrm{Cq}), 136.1(\mathrm{Cq}), 131.7$ $(\mathrm{CH}), 130.6(\mathrm{Cq}), 129.7(\mathrm{CH}), 128.7(\mathrm{Cq}), 128.6(\mathrm{CH}), 128.3(\mathrm{CH})$, $127.9(\mathrm{CH}), 127.7(\mathrm{CH}), 123.9(\mathrm{CH}), 122.3(\mathrm{Cq}), 112.3(\mathrm{CH}), 105.8$ $(\mathrm{CH}), 86.0(\mathrm{Cq}), 81.9(\mathrm{Cq}), 43.5\left(\mathrm{CH}_{2}\right), 37.5\left(\mathrm{CH}_{2}\right), 21.5\left(\mathrm{CH}_{3}\right)$. HRMS (ESI) $m / z:[\mathrm{M}+\mathrm{H}]^{+}$calcd for $\mathrm{C}_{27} \mathrm{H}_{24} \mathrm{NO}_{3} \mathrm{~S}^{+} 442.1471$; found 442.1463.

$\mathrm{N}$-(3-(4-Methoxyphenyl)prop-2-yn-1-yl)-4-methyl-N-((5phenylfuran-2-yl)methyl)benzenesulfonamide, $1 \mathrm{k}$. Synthesized according to the general procedure GP4 at rt with $p$-iodoanisole. White solid, $435 \mathrm{mg}$, 93\% yield, $130-132{ }^{\circ} \mathrm{C}$ (EtOAc). $R_{f} 0.24$ (9/1 PE/ EtOAc). ${ }^{1} \mathrm{H}$ NMR $\left(600 \mathrm{MHz}, \mathrm{CDCl}_{3}\right) \delta(\mathrm{ppm}): 7.81-7.79(\mathrm{~m}, 2 \mathrm{H})$, $7.59-7.57(\mathrm{~m}, 2 \mathrm{H}), 7.37-7.34(\mathrm{~m}, 2 \mathrm{H}), 7.27-7.24(\mathrm{~m}, 3 \mathrm{H}), 7.08-$ $7.05(\mathrm{~m}, 2 \mathrm{H}), 6.78-6.75(\mathrm{~m}, 2 \mathrm{H}), 6.56(\mathrm{~d}, 1 \mathrm{H}, J=3.3 \mathrm{~Hz}), 6.41(\mathrm{~d}, 1 \mathrm{H}$, $J=3.3 \mathrm{~Hz}), 4.55(\mathrm{~s}, 2 \mathrm{H}), 4.29(\mathrm{~s}, 2 \mathrm{H}), 3.80(\mathrm{~s}, 3 \mathrm{H}), 2.33(\mathrm{~s}, 3 \mathrm{H})$. ${ }^{13} \mathrm{C}\left\{{ }^{1} \mathrm{H}\right\}$ NMR $\left(150 \mathrm{MHz}, \mathrm{CDCl}_{3}\right) \delta(\mathrm{ppm}): 159.8(\mathrm{Cq}), 154.5(\mathrm{Cq})$, $148.4(\mathrm{Cq}), 143.6(\mathrm{Cq}), 136.2(\mathrm{Cq}), 133.2(\mathrm{CH}), 130.6(\mathrm{Cq}), 129.7$ $(\mathrm{CH}), 128.7(\mathrm{CH}), 127.9(\mathrm{CH}), 127.6(\mathrm{CH}), 123.9(\mathrm{CH}), 114.4(\mathrm{Cq})$, $113.9(\mathrm{CH}), 112.2(\mathrm{CH}), 105.8(\mathrm{CH}), 85.9(\mathrm{Cq}), 80.4(\mathrm{Cq}), 55.4$ $\left(\mathrm{CH}_{3}\right), 43.4\left(\mathrm{CH}_{2}\right), 37.5\left(\mathrm{CH}_{2}\right), 21.6\left(\mathrm{CH}_{3}\right)$. HRMS (ESI) $\mathrm{m} / z:[\mathrm{M}+$ $\mathrm{Na}]^{+}$calcd for $\mathrm{C}_{28} \mathrm{H}_{25} \mathrm{NO}_{4} \mathrm{SNa}^{+}$494.1397; found 494.1385.

4-Methyl- $N$-(3-phenylprop-2-yn-1-yl)- $N$-((5-(thiophen-2-yl)furan-2-yl)methyl)benzenesulfonamide, 11. Synthesized according to the general procedure GP4 at rt with PhI. Yellow solid, $318 \mathrm{mg}$, $71 \%$, mp 90-92 ${ }^{\circ} \mathrm{C}\left(\mathrm{Et}_{2} \mathrm{O}\right) \cdot R_{f} 0.23$ (9/1 PE/EtOAc). ${ }^{1} \mathrm{H}$ NMR $(600$ $\left.\mathrm{MHz}, \mathrm{CDCl}_{3}\right) \delta(\mathrm{ppm}): 7.80(\mathrm{~d}, 2 \mathrm{H}, J=8.3 \mathrm{~Hz}), 7.31-7.28(\mathrm{~m}, 1 \mathrm{H})$, $7.27-7.23(\mathrm{~m}, 4 \mathrm{H}), 7.21(\mathrm{dd}, 1 \mathrm{H}, J=5.0,1.0 \mathrm{~Hz}), 7.19(\mathrm{dd}, 1 \mathrm{H}, J=3.6$, $1.0 \mathrm{~Hz}), 7.14-7.11(\mathrm{~m}, 2 \mathrm{H}), 7.01(\mathrm{dd}, 1 \mathrm{H}, J=5.0,3.6 \mathrm{~Hz}), 6.41(\mathrm{~d}, 1 \mathrm{H}$, $J=3.3 \mathrm{~Hz}), 6.38(\mathrm{~d}, 1 \mathrm{H}, J=3.4 \mathrm{~Hz}), 4.53(\mathrm{~s}, 2 \mathrm{H}), 4.30(\mathrm{~s}, 2 \mathrm{H}), 2.32(\mathrm{~s}$, 3H). ${ }^{13} \mathrm{C}\left\{{ }^{1} \mathrm{H}\right\}$ NMR $\left(150 \mathrm{MHz}, \mathrm{CDCl}_{3}\right) \delta(\mathrm{ppm}): 150.0(\mathrm{Cq}), 148.0$ $(\mathrm{Cq}), 143.8(\mathrm{Cq}), 136.0(\mathrm{Cq}), 133.4(\mathrm{Cq}), 131.7(\mathrm{CH}), 129.7(\mathrm{CH})$, $128.6(\mathrm{CH}), 128.3(\mathrm{CH}), 127.9(\mathrm{CH}), 127.2(\mathrm{CH}), 124.5(\mathrm{CH}), 123.0$ $(\mathrm{CH}), 122.3(\mathrm{Cq}), 112.2(\mathrm{CH}), 105.8(\mathrm{CH}), 86.1(\mathrm{Cq}), 81.8(\mathrm{Cq})$, $43.4\left(\mathrm{CH}_{2}\right), 37.4\left(\mathrm{CH}_{2}\right), 21.6\left(\mathrm{CH}_{3}\right)$. HRMS (ESI) $\mathrm{m} / z:[\mathrm{M}+\mathrm{Na}]^{+}$ calcd for $\mathrm{C}_{25} \mathrm{H}_{21} \mathrm{NO}_{3} \mathrm{~S}_{2} \mathrm{Na}^{+} 470.0855$; found 470.0840 .

$\mathrm{N}$-([2,2'-Bifuran]-5-ylmethyl)-4-methyl- $\mathrm{N}$-(3-phenylprop-2yn-1-yl)benzenesulfonamide, $1 \mathrm{~m}$. Synthesized according to the general procedure GP4 at rt with PhI. Yellow solid, $303 \mathrm{mg}, 70 \%$ yield, $\mathrm{mp} 84-86^{\circ} \mathrm{C}\left(\mathrm{CHCl}_{3}\right) \cdot R_{f} 0.54(7 / 3 \mathrm{DCM} / \mathrm{PE}) .{ }^{1} \mathrm{H} \mathrm{NMR}(600 \mathrm{MHz}$, $\left.\mathrm{CDCl}_{3}\right) \delta(\mathrm{ppm}): 7.29(\mathrm{~d}, 2 \mathrm{H}, J=8.3 \mathrm{~Hz}), 7.40-7.39(\mathrm{~m}, 1 \mathrm{H}), 7.31-$ $7.28(\mathrm{~m}, 1 \mathrm{H}), 7.27-7.23(\mathrm{~m}, 4 \mathrm{H}), 7.11-7.09(\mathrm{~m}, 2 \mathrm{H}), 6.49(\mathrm{~d}, 1 \mathrm{H}, J=$ $3.4 \mathrm{~Hz}), 6.46(\mathrm{~d}, 1 \mathrm{H}, J=3.3 \mathrm{~Hz}), 6.44(\mathrm{dd}, 1 \mathrm{H}, J=3.4,1.8 \mathrm{~Hz}), 6.39(\mathrm{~d}$, $1 \mathrm{H}, J=3.3 \mathrm{~Hz}), 4.53(\mathrm{~s}, 2 \mathrm{H}), 4.38(\mathrm{~s}, 2 \mathrm{H}), 2.33(\mathrm{~s}, 3 \mathrm{H}) .{ }^{13} \mathrm{C}\left\{{ }^{1} \mathrm{H}\right\} \mathrm{NMR}$ $\left(150 \mathrm{MHz}, \mathrm{CDCl}_{3}\right) \delta(\mathrm{ppm}): 148.1(\mathrm{Cq}), 147.1(\mathrm{Cq}), 146.3(\mathrm{Cq})$, $143.8(\mathrm{Cq}), 142.0(\mathrm{CH}), 136.0(\mathrm{Cq}), 131.7(\mathrm{CH}), 129.7(\mathrm{CH}), 128.6$ $(\mathrm{CH}), 128.3(\mathrm{CH}), 128.0(\mathrm{CH}), 122.3(\mathrm{Cq}), 112.0(\mathrm{CH}), 111.5(\mathrm{CH})$, $105.9(\mathrm{CH}), 105.8(\mathrm{CH}), 86.1(\mathrm{Cq}), 81.7(\mathrm{Cq}), 43.4\left(\mathrm{CH}_{2}\right), 37.4$ $\left(\mathrm{CH}_{2}\right), 21.6\left(\mathrm{CH}_{3}\right)$. HRMS (ESI) $m / z:[\mathrm{M}+\mathrm{Na}]^{+}$calcd for $\mathrm{C}_{25} \mathrm{H}_{21} \mathrm{NO}_{4} \mathrm{SNa}^{+} 454.1083$; found 454.1078 .

2-Methyl-5-(((3-phenylprop-2-yn-1-yl)oxy)methyl)furan, $1 \mathrm{n}$. Synthesized according to the general procedure GP4 at $\mathrm{rt}$ with PhI. Colorless oil, $166 \mathrm{mg}, 74 \%$ yield. $R_{f} 0.21$ (99/1 PE/EtOAc). ${ }^{1} \mathrm{H}$ NMR $\left(600 \mathrm{MHz}, \mathrm{CDCl}_{3}\right) \delta(\mathrm{ppm}): 7.48-7.44(\mathrm{~m}, 2 \mathrm{H}), 7.34-7.30(\mathrm{~m}, 3 \mathrm{H})$, $6.28(\mathrm{~d}, 1 \mathrm{H}, J=3.0 \mathrm{~Hz}), 5.94(\mathrm{dq}, 1 \mathrm{H}, J=3.0,1.0 \mathrm{~Hz}), 4.56(\mathrm{~s}, 2 \mathrm{H})$, $4.39(\mathrm{~s}, 2 \mathrm{H}), 2.30(\mathrm{~d}, 3 \mathrm{H}, J=0.8 \mathrm{~Hz}) .{ }^{13} \mathrm{C}\left\{{ }^{1} \mathrm{H}\right\}$ NMR $(150 \mathrm{MHz}$, $\left.\mathrm{CDCl}_{3}\right) \delta(\mathrm{ppm}): 153.1(\mathrm{Cq}), 149.2(\mathrm{Cq}), 131.9(\mathrm{CH}), 128.6(\mathrm{CH})$, $128.4(\mathrm{CH}), 122.8(\mathrm{Cq}), 111.2(\mathrm{CH}), 106.4(\mathrm{CH}), 86.6(\mathrm{Cq}), 85.0$ (Cq), $63.5\left(\mathrm{CH}_{2}\right), 57.6\left(\mathrm{CH}_{2}\right), 13.7\left(\mathrm{CH}_{3}\right)$. HRMS (ESI) $m / z:[\mathrm{M}+$ $\mathrm{H}]^{+}$calcd for $\mathrm{C}_{15} \mathrm{H}_{15} \mathrm{O}_{2}^{+} 227.1067$; found 227.1067.

2-(((3-(4-Methoxyphenyl)prop-2-yn-1-yl)oxy)methyl)-5methylfuran, 10. Synthesized according to the general procedure GP4 at rt with $p$-iodoanisole. Colorless oil, $170 \mathrm{mg}, 66 \%$ yield. $R_{f} 0.28$ (96/4 PE/EtOAc). ${ }^{1} \mathrm{H}$ NMR (600 MHz, $\left.\mathrm{CDCl}_{3}\right) \delta$ (ppm): 7.41-7.38 $(\mathrm{m}, 2 \mathrm{H}), 6.86-6.83(\mathrm{~m}, 2 \mathrm{H}), 6.27(\mathrm{~d}, 1 \mathrm{H}, J=3.0 \mathrm{~Hz}), 5.93(\mathrm{dd}, 1 \mathrm{H}, J=$ 3.1, $1.0 \mathrm{~Hz}), 4.55(\mathrm{~s}, 2 \mathrm{H}), 4.38(\mathrm{~s}, 2 \mathrm{H}), 3.81(\mathrm{~s}, 3 \mathrm{H}), 2.29(\mathrm{~s}, 3 \mathrm{H})$. ${ }^{13} \mathrm{C}\left\{{ }^{1} \mathrm{H}\right\} \operatorname{NMR}\left(150 \mathrm{MHz}, \mathrm{CDCl}_{3}\right) \delta(\mathrm{ppm}): 159.8(\mathrm{Cq}), 153.1(\mathrm{Cq})$,
$149.3(\mathrm{Cq}), 133.4(\mathrm{CH}), 114.9(\mathrm{Cq}), 114.0(\mathrm{CH}), 111.2(\mathrm{CH}), 106.4$ $(\mathrm{CH}), 86.6(\mathrm{Cq}), 83.5(\mathrm{Cq}), 63.4\left(\mathrm{CH}_{2}\right), 57.7\left(\mathrm{CH}_{2}\right), 55.4\left(\mathrm{CH}_{3}\right)$, $13.8\left(\mathrm{CH}_{3}\right)$. HRMS (ESI) $\mathrm{m} / z$ : $[\mathrm{M}+\mathrm{H}]^{+}$calcd for $\mathrm{C}_{16} \mathrm{H}_{17} \mathrm{O}_{3}^{+}$ 257.1172; found 257.1175.

Methyl 4-(3-((5-Methylfuran-2-yl)methoxy)prop-1-yn-1-yl)benzoate, 1p. Synthesized according to the general procedure GP4 at $\mathrm{rt}$ with methyl $p$-iodobenzoate. White solid, $211 \mathrm{mg}, 75 \%$ yield, $\mathrm{mp}$ $45-47^{\circ} \mathrm{C}\left(\mathrm{Et}_{2} \mathrm{O}\right) . R_{f} 0.15$ (96/4 PE/EtOAc). ${ }^{1} \mathrm{H}$ NMR $(600 \mathrm{MHz}$, $\left.\mathrm{CDCl}_{3}\right) \delta(\mathrm{ppm}): 8.00-7.98(\mathrm{~m}, 2 \mathrm{H}), 7.52-7.50(\mathrm{~m}, 2 \mathrm{H}), 6.28(\mathrm{~d}, 1 \mathrm{H}$, $J=3.1 \mathrm{~Hz}), 5.94(\mathrm{dq}, 1 \mathrm{H}, J=3.0,1.1 \mathrm{~Hz}), 4.56(\mathrm{~s}, 2 \mathrm{H}), 4.40(\mathrm{~s}, 2 \mathrm{H})$, $3.92(\mathrm{~s}, 3 \mathrm{H}), 2.30(\mathrm{~d}, 3 \mathrm{H}, J=1.0 \mathrm{~Hz}) .{ }^{13} \mathrm{C}\left\{{ }^{1} \mathrm{H}\right\} \mathrm{NMR}(150 \mathrm{MHz}$, $\left.\mathrm{CDCl}_{3}\right) \delta(\mathrm{ppm}): 166.6(\mathrm{Cq}), 153.2(\mathrm{Cq}), 149.1(\mathrm{Cq}), 131.8(\mathrm{CH})$ $129.9(\mathrm{Cq}), 129.6(\mathrm{CH}), 127.5(\mathrm{Cq}), 111.4(\mathrm{CH}), 106.5(\mathrm{CH}), 88.1$ $(\mathrm{Cq}), 85.9(\mathrm{Cq}), 63.7\left(\mathrm{CH}_{2}\right), 57.5\left(\mathrm{CH}_{2}\right), 52.4\left(\mathrm{CH}_{3}\right), 13.8\left(\mathrm{CH}_{3}\right)$. HRMS (ESI) $m / z:[\mathrm{M}+\mathrm{Na}]^{+}$calcd for $\mathrm{C}_{17} \mathrm{H}_{16} \mathrm{O}_{4} \mathrm{Na}^{+} 307.0941$; found 307.0940

2-Methyl-5-(1-((3-phenylprop-2-yn-1-yl)oxy)propyl)furan, 1q. Synthesized according to the general procedure GP4 at rt with PhI. Colorless oil, $193 \mathrm{mg}, 76 \%$ yield. $R_{f} 0.34$ (99/1 PE/EtOAc). ${ }^{1} \mathrm{H}$ NMR $\left(600 \mathrm{MHz}, \mathrm{CDCl}_{3}\right) \delta(\mathrm{ppm}): 7.45-7.42(\mathrm{~m}, 2 \mathrm{H}), 7.32-7.29(\mathrm{~m}, 3 \mathrm{H})$, $6.22(\mathrm{~d}, 1 \mathrm{H}, J=3.0 \mathrm{~Hz}), 5.93-5.91(\mathrm{~m}, 1 \mathrm{H}), 4.42(\mathrm{t}, 1 \mathrm{H}, J=7.1 \mathrm{~Hz})$, $4.36(\mathrm{~d}, 1 \mathrm{H}, J=15.8 \mathrm{~Hz}), 4.21(\mathrm{~d}, 1 \mathrm{H}, J=15.8 \mathrm{~Hz}), 2.28(\mathrm{~d}, 3 \mathrm{H}, J=0.9$ $\mathrm{Hz}), 2.00-1.85(\mathrm{~m}, 2 \mathrm{H}), 0.93(\mathrm{t}, 3 \mathrm{H}, J=7.5 \mathrm{~Hz}) .{ }^{13} \mathrm{C}\left\{{ }^{1} \mathrm{H}\right\} \mathrm{NMR}(150$ $\left.\mathrm{MHz}, \mathrm{CDCl}_{3}\right) \delta(\mathrm{ppm}): 152.3(\mathrm{Cq}), 151.6(\mathrm{Cq}), 131.8(\mathrm{CH}), 128.4$ $(\mathrm{CH}), 128.3(\mathrm{CH}), 122.9(\mathrm{Cq}), 109.9(\mathrm{CH}), 106.0(\mathrm{CH}), 85.9(\mathrm{Cq})$, $85.6(\mathrm{Cq}), 75.3(\mathrm{CH}), 56.3\left(\mathrm{CH}_{2}\right), 27.1\left(\mathrm{CH}_{2}\right), 13.7\left(\mathrm{CH}_{3}\right), 10.3$ $\left(\mathrm{CH}_{3}\right)$. HRMS (ESI) m/z: $[\mathrm{M}+\mathrm{Na}]^{+}$calcd for $\mathrm{C}_{17} \mathrm{H}_{18} \mathrm{O}_{2} \mathrm{Na}^{+}$ 277.1199; found 277.1197.

2-(2,2-Dimethyl-1-((3-phenylprop-2-yn-1-yl)oxy)propyl)-5methylfuran, $1 \mathrm{r}$. Synthesized according to the general procedure GP4 at rt with PhI. Colorless oil, $165 \mathrm{mg}, 58 \%$ yield. $R_{f} 0.20(99 / 1 \mathrm{PE} /$ EtOAc). ${ }^{1} \mathrm{H}$ NMR $\left(600 \mathrm{MHz}, \mathrm{CDCl}_{3}\right) \delta(\mathrm{ppm}): 7.44-7.42(\mathrm{~m}, 2 \mathrm{H})$, $7.32-7.29(\mathrm{~m}, 3 \mathrm{H}), 6.16(\mathrm{~d}, 1 \mathrm{H}, J=3.0 \mathrm{~Hz}), 5.92(\mathrm{dq}, 1 \mathrm{H}, J=3.0,1.0$ $\mathrm{Hz}), 4.38(\mathrm{~d}, 1 \mathrm{H}, J=16.1 \mathrm{~Hz}), 4.19(\mathrm{~s}, 1 \mathrm{H}), 4.11(\mathrm{~d}, 1 \mathrm{H}, J=16.1 \mathrm{~Hz})$, $2.28(\mathrm{~s}, 3 \mathrm{H}), 0.98(\mathrm{~s}, 9 \mathrm{H}) .{ }^{13} \mathrm{C}\left\{{ }^{1} \mathrm{H}\right\} \mathrm{NMR}\left(150 \mathrm{MHz}, \mathrm{CDCl}_{3}\right) \delta(\mathrm{ppm})$ : $151.9(\mathrm{Cq}), 151.2(\mathrm{Cq}), 131.8(\mathrm{CH}), 128.4(\mathrm{CH}), 128.48(\mathrm{CH}), 123.1$ $(\mathrm{Cq}), 110.3(\mathrm{CH}), 105.9(\mathrm{CH}), 85.9(\mathrm{Cq}), 82.2(\mathrm{Cq}), 56.9\left(\mathrm{CH}_{2}\right)$, $35.4(\mathrm{Cq}), 26.4\left(\mathrm{CH}_{3}\right), 13.8\left(\mathrm{CH}_{3}\right)$. HRMS (ESI) $\mathrm{m} / z:[\mathrm{M}+\mathrm{Na}]^{+}$ calcd for $\mathrm{C}_{19} \mathrm{H}_{22} \mathrm{O}_{2} \mathrm{Na}^{+}$305.1512; found 305.1500.

$N$-(Furan-2-ylmethyl)-4-methyl- $N$-(3-phenylprop-2-yn-1-yl)benzenesulfonamide, $1 \mathrm{~s}$. Synthesized according to the general procedure GP4 at rt with PhI. White solid, $280 \mathrm{mg}$, 77\% yield, mp 68$70{ }^{\circ} \mathrm{C}\left(\mathrm{Et}_{2} \mathrm{O}\right) \cdot R_{f} 0.18(95 / 5 \mathrm{PE} / \mathrm{EtOAc}) .{ }^{1} \mathrm{H}$ NMR $\left(600 \mathrm{MHz}, \mathrm{CDCl}_{3}\right)$ $\delta(\mathrm{ppm}): 7.79(\mathrm{~d}, 2 \mathrm{H}, J=8.3 \mathrm{~Hz}), 7.38(\mathrm{q}, 1 \mathrm{H}, J=0.9 \mathrm{~Hz}), 7.31-7.23$ (m, $5 \mathrm{H}), 7.11-7.08(\mathrm{~m}, 2 \mathrm{H}), 6.35-6.32(\mathrm{~m}, 2 \mathrm{H}), 4.48(\mathrm{~s}, 2 \mathrm{H}), 4.24(\mathrm{~s}$, $2 \mathrm{H}), 2.35(\mathrm{~s}, 3 \mathrm{H}) .{ }^{13} \mathrm{C}\left\{{ }^{1} \mathrm{H}\right\} \mathrm{NMR}\left(150 \mathrm{MHz}, \mathrm{CDCl}_{3}\right) \delta(\mathrm{ppm}): 148.9$ $(\mathrm{Cq}), 143.7(\mathrm{Cq}), 143.2(\mathrm{CH}), 136.1(\mathrm{Cq}), 131.7(\mathrm{CH}), 129.7(\mathrm{CH})$, $128.6(\mathrm{CH}), 128.3(\mathrm{CH}), 128.0(\mathrm{CH}), 122.3(\mathrm{Cq}), 110.6(\mathrm{CH}), 110.1$ $(\mathrm{CH}), 86.0(\mathrm{Cq}), 81.7(\mathrm{Cq}), 43.2\left(\mathrm{CH}_{2}\right), 37.3\left(\mathrm{CH}_{2}\right), 21.6\left(\mathrm{CH}_{3}\right)$. HRMS (ESI) $m / z:[\mathrm{M}+\mathrm{Na}]^{+}$calcd for $\mathrm{C}_{21} \mathrm{H}_{19} \mathrm{NO}_{3} \mathrm{SNa}^{+}$388.0978; found 388.0974.

General Procedure for Gold(I)-Catalyzed Synthesis of Dihydropyridinones and Pyranones (GP5). A solution of the furan-yne substrate (1.0 equiv, $0.2 \mathrm{mmol}$ ) and 4-nitropyridine $\mathrm{N}$-oxide (1.2 equiv) in DCE (0.1 M with respect to the furan-yne) was prepared, then $\left[(\operatorname{IPr}) \mathrm{Au}\left(\mathrm{NTf}_{2}\right)\right]$ (0.05 equiv) was added, and the mixture was stirred at room temperature for $20 \mathrm{~h}$. Then, $\mathrm{MsOH}$ (5.0 equiv) was added, and the mixture was stirred at room temperature for $1 \mathrm{~h}$. A few drops of $\mathrm{Et}_{3} \mathrm{~N}$ were added, and the solvent was evaporated under reduced pressure. The crude product was purified by flash column chromatography to afford pure dihydropyridinones or pyranones $\mathbf{2}$ as the product.

(E)-5-(3-Oxobut-1-en-1-yl)-4-phenyl-1-tosyl-1,6-dihydropyridin-3(2H)-one, $2 \mathrm{a}$. Synthesized according to the general procedure GP5. Yellow solid, $57 \mathrm{mg}, 72 \%$ yield, $\mathrm{mp} 176-177{ }^{\circ} \mathrm{C}$ ( $\mathrm{MeCN}$, decomposition). The reaction was also repeated at $1.3 \mathrm{mmol}$ scale, obtaining $367 \mathrm{mg}$ (71\% yield) of product. $R_{f} 0.21(75 / 25 \mathrm{PE} /$ EtOAc). ${ }^{1} \mathrm{H}$ NMR $\left(600 \mathrm{MHz}, \mathrm{CDCl}_{3}\right) \delta(\mathrm{ppm}): 7.73-7.69(\mathrm{~m}, 2 \mathrm{H})$, $7.39-7.34(\mathrm{~m}, 5 \mathrm{H}), 7.03(\mathrm{~d}, 1 \mathrm{H}, J=16.6 \mathrm{~Hz}), 6.87-6.84(\mathrm{~m}, 2 \mathrm{H}), 6.46$ $(\mathrm{d}, 1 \mathrm{H}, J=16.6 \mathrm{~Hz}), 4.31(\mathrm{~s}, 2 \mathrm{H}), 4.04(\mathrm{~s}, 2 \mathrm{H}), 2.44(\mathrm{~s}, 3 \mathrm{H}), 2.19$ (s, 
$3 \mathrm{H}) .{ }^{13} \mathrm{C}\left\{{ }^{1} \mathrm{H}\right\} \mathrm{NMR}\left(150 \mathrm{MHz}, \mathrm{CDCl}_{3}\right) \delta$ (ppm): 197.3 (Cq), 190.4 $(\mathrm{Cq}), 144.9(\mathrm{Cq}), 144.1(\mathrm{Cq}), 141.4(\mathrm{Cq}), 137.9(\mathrm{CH}), 133.4(\mathrm{Cq})$, $132.2(\mathrm{CH}), 131.3(\mathrm{Cq}), 130.5(\mathrm{CH}), 130.4(\mathrm{CH}), 129.2(\mathrm{CH}), 128.3$ $(\mathrm{CH}), 127.8(\mathrm{CH}), 53.3\left(\mathrm{CH}_{2}\right), 45.1\left(\mathrm{CH}_{2}\right), 28.0\left(\mathrm{CH}_{3}\right), 21.7\left(\mathrm{CH}_{3}\right)$. HRMS (ESI) $m / z:[\mathrm{M}+\mathrm{H}]^{+}$calcd for $\mathrm{C}_{22} \mathrm{H}_{22} \mathrm{NO}_{4} \mathrm{~S}^{+} 396.1264$; found 396.1262 .

(E)-4-(4-Methoxyphenyl)-5-(3-oxobut-1-en-1-yl)-1-tosyl-1,6dihydropyridin-3(2H)-one, $2 \mathrm{~b}$. Synthesized according to the general procedure GP5. Yellow solid, $64 \mathrm{mg}, 75 \%$ yield, mp $150-153{ }^{\circ} \mathrm{C}$ (decomposition). $R_{f} 0.24$ (7/3 PE/EtOAc). ${ }^{1} \mathrm{H}$ NMR $(600 \mathrm{MHz}$, $\left.\mathrm{CDCl}_{3}\right) \delta(\mathrm{ppm}): 7.69(\mathrm{~d}, 2 \mathrm{H}, J=8.3 \mathrm{~Hz}), 7.35(\mathrm{~d}, 2 \mathrm{H}, J=8.1 \mathrm{~Hz}), 7.09$ $(\mathrm{d}, 1 \mathrm{H}, J=16.6 \mathrm{~Hz}), 6.90-6.87(\mathrm{~m}, 2 \mathrm{H}), 6.82-6.79(\mathrm{~m}, 2 \mathrm{H}), 6.45(\mathrm{~d}$, $1 \mathrm{H}, J=16.6 \mathrm{~Hz}), 4.29(\mathrm{~s}, 2 \mathrm{H}), 4.03(\mathrm{~s}, 2 \mathrm{H}), 3.83(\mathrm{~s}, 3 \mathrm{H}), 2.44(\mathrm{~s}, 3 \mathrm{H})$, $2.22(\mathrm{~s}, 3 \mathrm{H}) .{ }^{13} \mathrm{C}\left\{{ }^{1} \mathrm{H}\right\}$ NMR $\left(150 \mathrm{MHz}, \mathrm{CDCl}_{3}\right) \delta(\mathrm{ppm}): 197.4(\mathrm{Cq})$, $190.7(\mathrm{Cq}), 160.3(\mathrm{Cq}), 144.8(\mathrm{Cq}), 143.5(\mathrm{Cq}), 140.9(\mathrm{Cq}), 138.2$ $(\mathrm{CH}), 133.3(\mathrm{Cq}), 132.0(\mathrm{CH}), 131.8(\mathrm{CH}), 130.4(\mathrm{CH}), 127.8(\mathrm{CH})$, $123.3(\mathrm{Cq}), 113.8(\mathrm{CH}), 55.4\left(\mathrm{CH}_{3}\right), 53.3\left(\mathrm{CH}_{2}\right), 45.2\left(\mathrm{CH}_{2}\right), 28.1$ $\left(\mathrm{CH}_{3}\right), 21.7\left(\mathrm{CH}_{3}\right)$. HRMS (ESI) $m / z:[\mathrm{M}+\mathrm{Na}]^{+} \mathrm{C}_{23} \mathrm{H}_{23} \mathrm{NO}_{5} \mathrm{SNa}^{+}$ 448.1189; found 448.1094.

(E)-4-(4-Hydroxyphenyl)-5-(3-oxobut-1-en-1-yl)-1-tosyl-1,6dihydropyridin-3 $(2 \mathrm{H})$-one, $2 \mathrm{c}$. Synthesized according to the general procedure GP5. Yellow solid, $42 \mathrm{mg}$, 52\% yield, mp $170-174{ }^{\circ} \mathrm{C}$ (decomposition). $R_{f} 0.25$ (9/1 DCM/EtOAc). ${ }^{1} \mathrm{H}$ NMR $(600 \mathrm{MHz}$, $\left.\mathrm{CD}_{3} \mathrm{OD}\right) \delta(\mathrm{ppm}): 7.74-7.72(\mathrm{~m}, 2 \mathrm{H}), 7.42(\mathrm{~d}, 2 \mathrm{H}, J=7.9 \mathrm{~Hz}), 7.07$ $(\mathrm{d}, 1 \mathrm{H}, J=16.4 \mathrm{~Hz}), 6.78-6.75(\mathrm{~m}, 2 \mathrm{H}), 6.69(\mathrm{~d}, 1 \mathrm{H}, J=16.4 \mathrm{~Hz})$, 6.64-6.62 (m, 2H), $4.51(\mathrm{~s}, 2 \mathrm{H}), 4.17(\mathrm{~s}, 2 \mathrm{H}), 2.45(\mathrm{~s}, 3 \mathrm{H}), 2.24(\mathrm{~s}$, $3 \mathrm{H}), 2.19(\mathrm{~s}, 1 \mathrm{H}) \cdot{ }^{13} \mathrm{C}\left\{{ }^{1} \mathrm{H}\right\} \mathrm{NMR}\left(150 \mathrm{MHz}, \mathrm{CD}_{3} \mathrm{OD}\right) \delta(\mathrm{ppm}): 200.5$ $(\mathrm{Cq}), 193.7(\mathrm{Cq}), 160.2(\mathrm{Cq}), 146.9(\mathrm{Cq}), 145.9(\mathrm{Cq}), 143.0(\mathrm{Cq})$, $140.2(\mathrm{CH}), 136.9(\mathrm{Cq}), 134.1(\mathrm{CH}), 133.4(\mathrm{CH}), 132.3(\mathrm{CH}), 129.6$ $(\mathrm{CH}), 124.6(\mathrm{Cq}), 116.5(\mathrm{CH}), 55.2\left(\mathrm{CH}_{2}\right), 47.1\left(\mathrm{CH}_{2}\right), 29.1\left(\mathrm{CH}_{3}\right)$, $22.3\left(\mathrm{CH}_{3}\right)$. HRMS (ESI) $m / z:[\mathrm{M}+\mathrm{Na}]^{+}$calcd for $\mathrm{C}_{22} \mathrm{H}_{21} \mathrm{NO}_{5} \mathrm{SNa}^{+}$ 434.1033; found 434.1024.

(E)-5-(3-Oxobut-1-en-1-yl)-4-( -tolyl)-1-tosyl-1,6-dihydropyridin-3(2H)-one, 2d. Synthesized according to the general procedure GP5. Yellow solid, $71 \mathrm{mg}, 86 \%$ yield, mp $166-167{ }^{\circ} \mathrm{C}$ $\left(\mathrm{Et}_{2} \mathrm{O}\right) \cdot R_{f} 0.25(75 / 25 \mathrm{PE} / \mathrm{EtOAc}) .{ }^{1} \mathrm{H}$ NMR $\left(600 \mathrm{MHz}, \mathrm{CDCl}_{3}\right) \delta$ (ppm): $7.71-7.69(\mathrm{~m}, 2 \mathrm{H}), 7.35(\mathrm{~d}, 2 \mathrm{H}, J=8.0 \mathrm{~Hz}), 7.16(\mathrm{~d}, 2 \mathrm{H}, J=$ $7.8 \mathrm{~Hz}), 7.07(\mathrm{~d}, 1 \mathrm{H}, J=16.6 \mathrm{~Hz}), 6.75-6.72(\mathrm{~m}, 2 \mathrm{H}), 6.45(\mathrm{~d}, 1 \mathrm{H}, J=$ $16.5 \mathrm{~Hz}), 4.30(\mathrm{~s}, 2 \mathrm{H}), 4.04(\mathrm{~s}, 2 \mathrm{H}), 2.44(\mathrm{~s}, 3 \mathrm{H}), 2.36(\mathrm{~s}, 3 \mathrm{H}), 2.21(\mathrm{~s}$, $3 \mathrm{H}) .{ }^{13} \mathrm{C}\left\{{ }^{1} \mathrm{H}\right\}$ NMR $\left(150 \mathrm{MHz}, \mathrm{CDCl}_{3}\right) \delta(\mathrm{ppm}): 197.4(\mathrm{Cq}), 190.6$ $(\mathrm{Cq}), 144.8(\mathrm{Cq}), 143.7(\mathrm{Cq}), 141.4(\mathrm{Cq}), 139.3(\mathrm{Cq}), 138.1(\mathrm{CH})$, $133.4(\mathrm{Cq}), 131.9(\mathrm{CH}), 130.4(\mathrm{CH}), 129.1(\mathrm{CH}), 128.2(\mathrm{Cq}), 127.8$ $(\mathrm{CH}), 53.3\left(\mathrm{CH}_{2}\right), 45.1\left(\mathrm{CH}_{2}\right), 28.1\left(\mathrm{CH}_{3}\right), 21.7\left(\mathrm{CH}_{3}\right), 21.5\left(\mathrm{CH}_{3}\right)$. HRMS (ESI) $m / z$ : $[\mathrm{M}+\mathrm{H}]^{+}$calcd for $\mathrm{C}_{23} \mathrm{H}_{24} \mathrm{NO}_{4} \mathrm{~S}^{+} 410.1421$; found 410.1415 .

Methyl (E)-4-(3-Oxo-5-(3-oxobut-1-en-1-yl)-1-tosyl-1,2,3,6tetrahydropyridin-4-yl)benzoate, 2e. Synthesized according to the general procedure GP5. Light orange solid, $52 \mathrm{mg}$, $57 \%$ yield, $\mathrm{mp}$ $186-191{ }^{\circ} \mathrm{C}$ (decomposition). $R_{f} 0.21$ (65/35 PE/EtOAc). ${ }^{1} \mathrm{H}$ NMR $\left(600 \mathrm{MHz}, \mathrm{CDCl}_{3}\right) \delta(\mathrm{ppm}): 8.04-8.02(\mathrm{~m}, 2 \mathrm{H}), 7.71-7.69(\mathrm{~m}, 2 \mathrm{H})$, $7.36(\mathrm{~d}, 2 \mathrm{H}, J=8.0 \mathrm{~Hz}), 6.95(\mathrm{~d}, 1 \mathrm{H}, J=16.6 \mathrm{~Hz})$ superimposed to 6.94-6.92 (m, 2H), $6.50(\mathrm{~d}, 1 \mathrm{H}, J=16.5 \mathrm{~Hz}), 4.34(\mathrm{~s}, 2 \mathrm{H}), 4.06(\mathrm{~s}$, $2 \mathrm{H}), 3.93(\mathrm{~s}, 3 \mathrm{H}), 2.45(\mathrm{~s}, 3 \mathrm{H}), 2.20(\mathrm{~s}, 3 \mathrm{H}) .{ }^{13} \mathrm{C}\left\{{ }^{1} \mathrm{H}\right\}$ NMR $(150$ $\left.\mathrm{MHz}, \mathrm{CDCl}_{3}\right) \delta(\mathrm{ppm}): 197.0(\mathrm{Cq}), 190.0(\mathrm{Cq}), 166.6(\mathrm{Cq}), 145.0$ $(\mathrm{Cq}), 144.8(\mathrm{Cq}), 140.4(\mathrm{Cq}), 137.0(\mathrm{CH}), 136.0(\mathrm{Cq}), 133.4(\mathrm{Cq})$, $132.7(\mathrm{CH}), 130.7(\mathrm{Cq}), 130.6(\mathrm{CH}), 130.5(\mathrm{CH}), 129.5(\mathrm{CH}), 127.8$ $(\mathrm{CH}), 53.2\left(\mathrm{CH}_{2}\right), 52.5\left(\mathrm{CH}_{3}\right), 45.1\left(\mathrm{CH}_{2}\right), 28.2\left(\mathrm{CH}_{3}\right), 21.7\left(\mathrm{CH}_{3}\right)$. HRMS (ESI) $m / z$ : $[\mathrm{M}+\mathrm{H}]^{+}$calcd for $\mathrm{C}_{24} \mathrm{H}_{24} \mathrm{NO}_{6} \mathrm{~S}^{+} 454.1319$; found 454.1307.

(E)-4-(4-Acetylphenyl)-5-(3-oxobut-1-en-1-yl)-1-tosyl-1,6dihydropyridin-3(2H)-one, $2 \mathrm{f}$. Synthesized according to the general procedure GP5. White solid, $37 \mathrm{mg}$, $43 \%$ yield, mp $161-164{ }^{\circ} \mathrm{C}$ (decomposition). $R_{f} 0.24$ (6/4 PE/EtOAc). ${ }^{1} \mathrm{H}$ NMR $(600 \mathrm{MHz}$, $\left.\mathrm{CDCl}_{3}\right) \delta(\mathrm{ppm}): 7.96-7.93(\mathrm{~m}, 2 \mathrm{H}), 7.71(\mathrm{~d}, 2 \mathrm{H}, J=8.3 \mathrm{~Hz}), 7.37(\mathrm{~d}$, $2 \mathrm{H}, J=8.0 \mathrm{~Hz}), 6.96(\mathrm{~d}, 1 \mathrm{H}, J=16.4 \mathrm{~Hz})$ superimposed to $6.98-6.95$ $(\mathrm{m}, 2 \mathrm{H}), 6.51(\mathrm{~d}, 1 \mathrm{H}, J=16.4 \mathrm{~Hz}), 4.34(\mathrm{~s}, 2 \mathrm{H}), 4.06(\mathrm{~s}, 2 \mathrm{H}), 2.62(\mathrm{~s}$, $3 \mathrm{H}), 2.45(\mathrm{~s}, 3 \mathrm{H}), 2.22(\mathrm{~s}, 3 \mathrm{H}) \cdot{ }^{13} \mathrm{C}\left\{{ }^{1} \mathrm{H}\right\} \operatorname{NMR}\left(150 \mathrm{MHz}, \mathrm{CDCl}_{3}\right) \delta$ $(\mathrm{ppm}): 197.6(\mathrm{Cq}), 196.9(\mathrm{Cq}), 190.0(\mathrm{Cq}), 145.0(\mathrm{Cq}), 144.8(\mathrm{Cq})$, $140.4(\mathrm{Cq}), 137.4(\mathrm{Cq}), 136.9(\mathrm{CH}), 136.2(\mathrm{Cq}), 133.4(\mathrm{Cq}), 132.7$ $(\mathrm{CH}), 130.8(\mathrm{CH}), 130.5(\mathrm{CH}), 128.2(\mathrm{CH}), 127.8(\mathrm{CH}), 53.2\left(\mathrm{CH}_{2}\right)$,
$45.1\left(\mathrm{CH}_{2}\right), 28.4\left(\mathrm{CH}_{3}\right), 26.8\left(\mathrm{CH}_{3}\right), 21.7\left(\mathrm{CH}_{3}\right)$. HRMS (ESI) $m / z$ : $[\mathrm{M}+\mathrm{Na}]^{+}$calcd for $\mathrm{C}_{24} \mathrm{H}_{23} \mathrm{NO}_{5} \mathrm{SNa}^{+} 460.1189$; found 460.1182 .

(E)-4-(4-Fluorophenyl)-5-(3-oxobut-1-en-1-yl)-1-tosyl-1,6dihydropyridin-3(2H)-one, $2 \mathrm{~g}$. Synthesized according to the general procedure GP5. Yellow solid, $40 \mathrm{mg}$, $48 \%$ yield, mp $149-153{ }^{\circ} \mathrm{C}$ (decomposition). $R_{f} 0.16$ (7/3 PE/EtOAc). ${ }^{1} \mathrm{H}$ NMR $(600 \mathrm{MHz}$, $\left.\mathrm{CDCl}_{3}\right) \delta(\mathrm{ppm}): 7.70(\mathrm{~d}, 2 \mathrm{H}, J=8.3 \mathrm{~Hz}), 7.36(\mathrm{~d}, 2 \mathrm{H}, J=7.9 \mathrm{~Hz})$, $7.08-7.05(\mathrm{~m}, 2 \mathrm{H}), 7.01(\mathrm{~d}, 1 \mathrm{H}, J=16.4 \mathrm{~Hz}), 6.87-6.83(\mathrm{~m}, 2 \mathrm{H}), 6.49$ $(\mathrm{d}, 1 \mathrm{H}, J=16.5 \mathrm{~Hz}), 4.31(\mathrm{~s}, 2 \mathrm{H}), 4.04(\mathrm{~s}, 2 \mathrm{H}), 2.44(\mathrm{~s}, 3 \mathrm{H}), 2.22(\mathrm{~s}$, $3 \mathrm{H}) .{ }^{13} \mathrm{C}\left\{{ }^{1} \mathrm{H}\right\}$ NMR $\left(150 \mathrm{MHz}, \mathrm{CDCl}_{3}\right) \delta(\mathrm{ppm}): 197.1(\mathrm{Cq}), 190.4$ $(\mathrm{Cq}), 163.2(\mathrm{Cq}, \mathrm{d}, J=250.1 \mathrm{~Hz}), 144.9(\mathrm{Cq}), 144.4(\mathrm{Cq}), 140.3(\mathrm{Cq})$, $137.4(\mathrm{CH}), 133.3(\mathrm{Cq}), 132.4(\mathrm{CH}, \mathrm{d}, J=8.7 \mathrm{~Hz}), 132.3(\mathrm{CH}), 130.4$ $(\mathrm{CH}), 127.8(\mathrm{CH}), 127.1(\mathrm{Cq}), 115.5(\mathrm{CH}, \mathrm{d}, J=21.5 \mathrm{~Hz}), 53.3$ $\left(\mathrm{CH}_{2}\right), 45.1\left(\mathrm{CH}_{2}\right), 28.3\left(\mathrm{CH}_{3}\right), 21.7\left(\mathrm{CH}_{3}\right) .{ }^{19} \mathrm{~F}$ NMR $(564 \mathrm{MHz}$, $\left.\mathrm{CDCl}_{3}\right) \delta(\mathrm{ppm}):(-111.4)-(-112.0)(\mathrm{m})$. HRMS (ESI) $\mathrm{m} / z$ : $[\mathrm{M}+$ $\mathrm{Na}]^{+}$calcd for $\mathrm{C}_{22} \mathrm{H}_{20} \mathrm{FNO}_{4} \mathrm{SNa}^{+} 436.0989$; found 436.0969.

(E)-5-(3-Oxobut-1-en-1-yl)-4-(thiophen-2-yl)-1-tosyl-1,6dihydropyridin-3(2H)-one, $2 \mathrm{~h}$. Synthesized according to the general procedure GP5. Yellow solid, $24 \mathrm{mg}, 30 \%$ yield, mp 146-148 $\left(\mathrm{Et}_{2} \mathrm{O}\right.$, decomposition). $R_{f} 0.23$ (7/3 PE/EtOAc). ${ }^{1} \mathrm{H}$ NMR $(600 \mathrm{MHz}$, $\left.\mathrm{CDCl}_{3}\right) \delta(\mathrm{ppm}): 7.70-7.67(\mathrm{~m}, 2 \mathrm{H}), 7.52(\mathrm{dd}, 1 \mathrm{H}, J=5.2,1.2 \mathrm{~Hz})$, $7.36(\mathrm{~d}, 1 \mathrm{H}, J=16.5 \mathrm{~Hz}), 7.32(\mathrm{~d}, 2 \mathrm{H}, J=8.0 \mathrm{~Hz}), 7.06(\mathrm{dd}, 1 \mathrm{H}, J=5.1$, $3.6 \mathrm{~Hz}), 6.81(\mathrm{dd}, 1 \mathrm{H}, J=3.6,1.2 \mathrm{~Hz}), 6.55(\mathrm{~d}, 1 \mathrm{H}, J=16.5 \mathrm{~Hz}), 4.31(\mathrm{~s}$, $2 \mathrm{H}), 4.1(\mathrm{~s}, 2 \mathrm{H}), 2.39(\mathrm{~s}, 3 \mathrm{H}), 2.29(\mathrm{~s}, 3 \mathrm{H}) .{ }^{13} \mathrm{C}\left\{{ }^{1} \mathrm{H}\right\} \mathrm{NMR}(150 \mathrm{MHz}$, $\left.\mathrm{CDCl}_{3}\right) \delta(\mathrm{ppm}): 197.3(\mathrm{Cq}), 189.9(\mathrm{Cq}), 144.9(\mathrm{Cq}), 143.9(\mathrm{Cq})$, $138.0(\mathrm{CH}), 134.4(\mathrm{Cq}), 133.2(\mathrm{Cq}), 132.4(\mathrm{CH}), 131.8(\mathrm{CH})$, 131.1(Cq), $130.5(\mathrm{CH}), 130.4(\mathrm{CH}), 127.7(\mathrm{CH}), 126.7(\mathrm{CH}), 53.3$ $\left(\mathrm{CH}_{2}\right), 45.7\left(\mathrm{CH}_{2}\right), 28.3\left(\mathrm{CH}_{3}\right), 21.7\left(\mathrm{CH}_{3}\right)$. HRMS (ESI) $\mathrm{m} / z$ : $[\mathrm{M}+$ $\mathrm{H}]^{+}$calcd for $\mathrm{C}_{20} \mathrm{H}_{20} \mathrm{NO}_{4} \mathrm{~S}_{2}{ }^{+} 402.0828$; found 402.0827 .

(E)-4-(Naphthalen-1-yl)-5-(3-oxobut-1-en-1-yl)-1-tosyl-1,6dihydropyridin-3(2H)-one, $2 \mathrm{i}$. Synthesized according to the general procedure GP5. Yellow solid, $65 \mathrm{mg}, 73 \%$ yield, $\mathrm{mp} 131-141{ }^{\circ} \mathrm{C}$ (decomposition). $R_{f} 0.16$ (7/3 PE/EtOAc). ${ }^{1} \mathrm{H}$ NMR $(600 \mathrm{MHz}$, $\left.\mathrm{CDCl}_{3}\right) \delta(\mathrm{ppm}): 7.89(\mathrm{~d}, 1 \mathrm{H}, J=8.3 \mathrm{~Hz}), 7.87(\mathrm{~d}, 1 \mathrm{H}, J=8.2 \mathrm{~Hz}), 7.77$ $(\mathrm{d}, 2 \mathrm{H}, J=8.4 \mathrm{~Hz}), 7.49-7.44(\mathrm{~m}, 2 \mathrm{H}), 7.42(\mathrm{~d}, 2 \mathrm{H}, J=7.9 \mathrm{~Hz}), 7.38-$ $7.35(\mathrm{~m}, 1 \mathrm{H}), 7.20(\mathrm{~d}, 1 \mathrm{H}, J=8.4 \mathrm{~Hz}), 6.91(\mathrm{dd}, 1 \mathrm{H}, J=7.0,1.0 \mathrm{~Hz})$, $6.79(\mathrm{~d}, 1 \mathrm{H}, J=16.6 \mathrm{~Hz}), 6.45(\mathrm{~d}, 1 \mathrm{H}, J=16.6 \mathrm{~Hz}), 4.42(\mathrm{~d}, 1 \mathrm{H}, J=17.2$ $\mathrm{Hz}), 4.34(\mathrm{~d}, 1 \mathrm{H}, J=17.4 \mathrm{~Hz}), 4.14(\mathrm{dd}, 1 \mathrm{H}, J=16.7,1.3 \mathrm{~Hz}), 4.04(\mathrm{dd}$, $1 \mathrm{H}, J=16.7,1.4 \mathrm{~Hz}), 2.49(\mathrm{~s}, 3 \mathrm{H}), 2.01(\mathrm{~s}, 3 \mathrm{H}) .{ }^{13} \mathrm{C}\left\{{ }^{1} \mathrm{H}\right\} \mathrm{NMR}(150$ $\left.\mathrm{MHz}, \mathrm{CDCl}_{3}\right) \delta(\mathrm{ppm}): 197.3(\mathrm{Cq}), 190.3(\mathrm{Cq}), 152.2(\mathrm{CH}), 146.4$ $(\mathrm{Cq}), 145.0(\mathrm{Cq}), 140.8(\mathrm{Cq}), 137.5(\mathrm{CH}), 133.6(\mathrm{Cq}), 133.0(\mathrm{Cq})$, $132.5(\mathrm{CH}), 132.0(\mathrm{Cq}), 130.5(\mathrm{CH}), 129.8(\mathrm{CH}), 129.4(\mathrm{Cq}), 128.8$ $(\mathrm{CH}), 128.4(\mathrm{CH}), 127.9(\mathrm{CH}), 126.8(\mathrm{CH}), 126.4(\mathrm{CH}), 125.1$ $(\mathrm{CH}), 125.0(\mathrm{CH}), 53.3\left(\mathrm{CH}_{2}\right), 44.9\left(\mathrm{CH}_{2}\right), 27.7\left(\mathrm{CH}_{3}\right), 21.8\left(\mathrm{CH}_{3}\right)$. HRMS (ESI) $m / z:[\mathrm{M}+\mathrm{H}]^{+}$calcd for $\mathrm{C}_{26} \mathrm{H}_{24} \mathrm{NO}_{4} \mathrm{~S}^{+} 446.1421$; found 446.1410 .

(E)-5-(3-0xo-3-phenylprop-1-en-1-yl)-4-phenyl-1-tosyl-1,6dihydropyridin-3(2H)-one, $2 \mathrm{j}$. Synthesized according to the general procedure GP5. Yellow solid, $57 \mathrm{mg}, 62 \%$ yield, mp $175-178{ }^{\circ} \mathrm{C}$ (decomposition). $R_{f} 0.23$ (8/2 PE/EtOAc). ${ }^{1} \mathrm{H}$ NMR $(600 \mathrm{MHz}$, $\left.\mathrm{CDCl}_{3}\right) \delta(\mathrm{ppm}): 7.92(\mathrm{~d}, 2 \mathrm{H}, J=7.2 \mathrm{~Hz}), 7.72(\mathrm{~d}, 2 \mathrm{H}, J=8.3 \mathrm{~Hz})$, 7.64-7.60 (m, 1H), $7.51(\mathrm{t}, 2 \mathrm{H}, J=7.8 \mathrm{~Hz}), 7.37-7.34(\mathrm{~m}, 5 \mathrm{H}), 7.29$ $(\mathrm{d}, 2 \mathrm{H}, J=1.3 \mathrm{~Hz}), 6.82(\mathrm{dd}, 2 \mathrm{H}, J=7.7,1.6 \mathrm{~Hz}), 4.49(\mathrm{~s}, 2 \mathrm{H}), 4.11(\mathrm{~s}$, 2H), $2.44(\mathrm{~s}, 3 \mathrm{H}) \cdot{ }^{13} \mathrm{C}\left\{{ }^{1} \mathrm{H}\right\}$ NMR $\left(150 \mathrm{MHz}, \mathrm{CDCl}_{3}\right) \delta$ (ppm): 190.6 $(\mathrm{Cq}), 189.3(\mathrm{Cq}), 144.9(\mathrm{Cq}), 144.1(\mathrm{Cq}), 141.5(\mathrm{Cq}), 139.2(\mathrm{CH})$, $137.2(\mathrm{Cq}), 133.7(\mathrm{CH}), 133.7(\mathrm{Cq}), 131.3(\mathrm{Cq}), 130.5(\mathrm{CH}), 130.5$ $(\mathrm{CH}), 129.1(\mathrm{CH}), 129.0(\mathrm{CH}), 128.7(\mathrm{CH}), 128.3(\mathrm{CH}), 127.8$ $(\mathrm{CH}), 127.7(\mathrm{CH}), 53.3\left(\mathrm{CH}_{2}\right), 45.2\left(\mathrm{CH}_{2}\right), 21.7\left(\mathrm{CH}_{3}\right)$. HRMS (ESI) $m / z:[\mathrm{M}+\mathrm{Na}]^{+}$calcd for $\mathrm{C}_{27} \mathrm{H}_{23} \mathrm{NO}_{4} \mathrm{SNa}^{+} 480.1240$; found 480.1220 .

(E)-4-(4-Methoxyphenyl)-5-(3-oxo-3-phenylprop-1-en-1-yl)1-tosyl-1,6-dihydropyridin-3(2H)-one, 2k. Synthesized according to the general procedure GP5. Yellow solid, $77 \mathrm{mg}, 78 \%$ yield, $\mathrm{mp} 159-$ $163{ }^{\circ} \mathrm{C}$ (decomposition). $R_{f} 0.40$ (99/1 DCM/EtOAc). ${ }^{1} \mathrm{H}$ NMR $(600$ $\left.\mathrm{MHz}, \mathrm{CDCl}_{3}\right) \delta(\mathrm{ppm}): 7.96-7.94(\mathrm{~m}, 2 \mathrm{H}), 7.73-7.70(\mathrm{~m}, 2 \mathrm{H}), 7.63$ $(\mathrm{tt}, 1 \mathrm{H}, J=7.1,1.2 \mathrm{~Hz}), 7.54-7.51(\mathrm{~m}, 2 \mathrm{H}), 7.36(\mathrm{~d}, 1 \mathrm{H}, J=16.0 \mathrm{~Hz})$ superimposed to $7.34(\mathrm{~d}, 2 \mathrm{H}, J=7.9 \mathrm{~Hz}), 7.28(\mathrm{~d}, 1 \mathrm{H}, J=16.0 \mathrm{~Hz})$, 6.88-6.86 (m, 2H), 6.79-6.77 (m, 2H), $4.47(\mathrm{~s}, 2 \mathrm{H}), 4.09(\mathrm{~s}, 2 \mathrm{H})$, $3.82(\mathrm{~s}, 3 \mathrm{H}), 2.43(\mathrm{~s}, 3 \mathrm{H}) .{ }^{13} \mathrm{C}\left\{{ }^{1} \mathrm{H}\right\} \operatorname{NMR}\left(150 \mathrm{MHz}, \mathrm{CDCl}_{3}\right) \delta(\mathrm{ppm})$ : $190.9(\mathrm{Cq}), 189.3(\mathrm{Cq}), 160.3(\mathrm{Cq}), 144.9(\mathrm{Cq}), 143.4(\mathrm{Cq}), 141.1$ (Cq), $139.7(\mathrm{CH}), 137.7(\mathrm{Cq}), 133.7(\mathrm{CH}), 133.7(\mathrm{Cq}), 132.1(\mathrm{CH})$, 
$130.5(\mathrm{CH}), 129.1(\mathrm{CH}), 128.8(\mathrm{CH}), 127.7(\mathrm{CH}), 127.3(\mathrm{CH}), 123.4$ (Cq), $113.8(\mathrm{CH}), 55.4\left(\mathrm{CH}_{3}\right), 53.4\left(\mathrm{CH}_{2}\right), 45.4\left(\mathrm{CH}_{2}\right), 21.7\left(\mathrm{CH}_{3}\right)$. HRMS (ESI) $\mathrm{m} / z$ : $[\mathrm{M}+\mathrm{Na}]^{+}$calcd for $\mathrm{C}_{28} \mathrm{H}_{25} \mathrm{NO}_{5} \mathrm{SNa}^{+}$510.1346; found 510.1324

(E)-5-(3-0xo-3-(thiophen-2-yl)prop-1-en-1-yl)-4-phenyl-1tosyl-1,6-dihydropyridin-3(2H)-one, 2l. Synthesized according to the general procedure GP5. Yellow solid, $58 \mathrm{mg}$, 63\% yield, mp 145$149{ }^{\circ} \mathrm{C}$ (decomposition). $R_{f} 0.24$ (7/3 PE/EtOAc). ${ }^{1} \mathrm{H}$ NMR (600 $\left.\mathrm{MHz}, \mathrm{CDCl}_{3}\right) \delta(\mathrm{ppm}): 7.82(\mathrm{dd}, 1 \mathrm{H}, J=3.8,1.0 \mathrm{~Hz}), 7.74(\mathrm{dd}, 1 \mathrm{H}, J=$ $4.9,1.0 \mathrm{~Hz}), 7.72-7.70(\mathrm{~m}, 2 \mathrm{H}), 7.37-7.32(\mathrm{~m}, 5 \mathrm{H})$ superimposed to $7.32(\mathrm{~d}, 1 \mathrm{H}, J=15.6 \mathrm{~Hz}), 7.21(\mathrm{dd}, 1 \mathrm{H}, J=4.9,3.9 \mathrm{~Hz}), 7.14(\mathrm{~d}, 1 \mathrm{H}, J=$ $15.8 \mathrm{~Hz}), 6.80-8.78(\mathrm{~m}, 2 \mathrm{H}), 4.49(\mathrm{~s}, 2 \mathrm{H}), 4.12(\mathrm{~s}, 2 \mathrm{H}), 2.44(\mathrm{~s}, 3 \mathrm{H})$. ${ }^{13} \mathrm{C}\left\{{ }^{1} \mathrm{H}\right\} \operatorname{NMR}\left(150 \mathrm{MHz}, \mathrm{CDCl}_{3}\right) \delta(\mathrm{ppm}): 190.6(\mathrm{Cq}), 180.9(\mathrm{Cq})$, $144.9(\mathrm{Cq}), 144.8(\mathrm{Cq}), 143.8(\mathrm{Cq}), 141.6(\mathrm{Cq}), 138.6(\mathrm{CH}), 135.4$ $(\mathrm{CH}), 133.8(\mathrm{Cq}), 132.8(\mathrm{CH}), 131.2(\mathrm{Cq}), 130.5(\mathrm{CH}), 130.5(\mathrm{CH})$, $129.2(\mathrm{CH}), 128.7(\mathrm{CH}), 128.3(\mathrm{CH}), 127.7(\mathrm{CH}), 127.5(\mathrm{CH}), 53.4$ $\left(\mathrm{CH}_{2}\right), 45.3\left(\mathrm{CH}_{2}\right), 21.7\left(\mathrm{CH}_{3}\right)$. HRMS (ESI) m/z: $[\mathrm{M}+\mathrm{Na}]^{+}$calcd for $\mathrm{C}_{25} \mathrm{H}_{21} \mathrm{NO}_{4} \mathrm{~S}_{2} \mathrm{Na}^{+}$486.0804; found 486.0784 .

(E)-5-(3-(Furan-2-yl)-3-oxoprop-1-en-1-yl)-4-phenyl-1-tosyl1,6-dihydropyridin-3(2H)-one, $2 \mathrm{~m}$. Synthesized according to the general procedure GP5. Yellow solid, $37 \mathrm{mg}$, 41\% yield, mp 159-162 ${ }^{\circ} \mathrm{C}$ (decomposition). $R_{f} 0.20$ (99/1 DCM/EtOAc). ${ }^{1} \mathrm{H}$ NMR (600 $\left.\mathrm{MHz}, \mathrm{CDCl}_{3}\right) \delta(\mathrm{ppm}): 7.73-7.71(\mathrm{~m}, 2 \mathrm{H}), 7.68(\mathrm{dd}, 1 \mathrm{H}, J=1.7,0.8$ $\mathrm{Hz}), 7.37-7.31(\mathrm{~m}, 7 \mathrm{H}), 7.22(\mathrm{~d}, 1 \mathrm{H}, J=16.1 \mathrm{~Hz}), 6.80-6.78(\mathrm{~m}, 2 \mathrm{H})$, $6.63(\mathrm{dd}, 1 \mathrm{H}, J=3.6,1.7 \mathrm{~Hz}), 4.50(\mathrm{~s}, 2 \mathrm{H}), 4.12(\mathrm{~s}, 2 \mathrm{H}), 2.44(\mathrm{~s}, 3 \mathrm{H})$. ${ }^{13} \mathrm{C}\left\{{ }^{1} \mathrm{H}\right\} \mathrm{NMR}\left(150 \mathrm{MHz}, \mathrm{CDCl}_{3}\right) \delta(\mathrm{ppm}): 190.6(\mathrm{Cq}), 176.7(\mathrm{Cq})$, $153.3(\mathrm{Cq}), 147.3(\mathrm{CH}), 144.9(\mathrm{Cq}), 143.9(\mathrm{Cq}), 141.6(\mathrm{Cq}), 138.5$ $(\mathrm{CH}), 133.9(\mathrm{Cq}), 131.2(\mathrm{Cq}), 130.6(\mathrm{CH}), 130.5(\mathrm{CH}), 129.2(\mathrm{CH})$, $128.3(\mathrm{CH}), 127.7(\mathrm{CH}), 127.2(\mathrm{CH}), 118.7(\mathrm{CH}), 113.2(\mathrm{CH}), 53.4$ $\left(\mathrm{CH}_{2}\right), 45.2\left(\mathrm{CH}_{2}\right), 21.7\left(\mathrm{CH}_{3}\right)$. HRMS (ESI) $\mathrm{m} / z:[\mathrm{M}+\mathrm{H}]^{+}$calcd for $\mathrm{C}_{25} \mathrm{H}_{22} \mathrm{NO}_{5} \mathrm{~S}^{+} 448.1213$; found 448.1202 .

(E)-5-(3-Oxobut-1-en-1-yl)-4-phenyl-2H-pyran-3(6H)-one, $2 n$. Synthesized according to the general procedure GP5. Yellow solid, $38 \mathrm{mg}, 78 \%$ yield, $\mathrm{mp} 104-106^{\circ} \mathrm{C}\left(\mathrm{Et}_{2} \mathrm{O}\right) . R_{f} 0.44$ (6/4 PE/EtOAc). ${ }^{1} \mathrm{H}$ NMR $\left(600 \mathrm{MHz}, \mathrm{CDCl}_{3}\right) \delta(\mathrm{ppm}): 7.46-7.41(\mathrm{~m}, 3 \mathrm{H}), 7.20-7.17$ $(\mathrm{m}, 2 \mathrm{H})$ superimposed to $7.15(\mathrm{~d}, 1 \mathrm{H}, J=16.7 \mathrm{~Hz}), 6.32(\mathrm{~d}, 1 \mathrm{H}, J=$ $16.7 \mathrm{~Hz}), 4.72(\mathrm{~s}, 2 \mathrm{H}), 4.34(\mathrm{~s}, 2 \mathrm{H}), 2.19(\mathrm{~s}, 3 \mathrm{H}) .{ }^{13} \mathrm{C}\left\{{ }^{1} \mathrm{H}\right\} \operatorname{NMR}(150$ $\left.\mathrm{MHz}, \mathrm{CDCl}_{3}\right) \delta(\mathrm{ppm}): 197.6(\mathrm{Cq}), 193.2(\mathrm{Cq}), 146.6(\mathrm{Cq}), 140.1$ $(\mathrm{Cq}), 137.5(\mathrm{CH}), 132.0(\mathrm{CH}), 131.3(\mathrm{Cq}), 130.7(\mathrm{CH}), 129.1(\mathrm{CH})$, $128.4(\mathrm{CH}), 72.5\left(\mathrm{CH}_{2}\right), 65.6\left(\mathrm{CH}_{2}\right), 27.6\left(\mathrm{CH}_{3}\right)$. HRMS (ESI) $\mathrm{m} / z$ : $[\mathrm{M}+\mathrm{H}]^{+}$calcd for $\mathrm{C}_{15} \mathrm{H}_{15} \mathrm{O}_{3}{ }^{+} 243.1016$; found 243.1017.

(E)-4-(4-Methoxyphenyl)-5-(3-oxobut-1-en-1-yl)-2H-pyran$3(6 H)$-one, 20. Synthesized according to the general procedure GP5. Yellow solid, $16 \mathrm{mg}$, 29\% yield. $R_{f} 0.35$ (75/25 PE/EtOAc). ${ }^{1} \mathrm{H}$ NMR $\left(600 \mathrm{MHz}, \mathrm{CDCl}_{3}\right) \delta(\mathrm{ppm}): 7.21(\mathrm{~d}, 1 \mathrm{H}, J=16.8 \mathrm{~Hz}), 7.15-7.12(\mathrm{~m}$, $2 \mathrm{H}), 6.98-6.95(\mathrm{~m}, 2 \mathrm{H}), 6.32(\mathrm{~d}, 1 \mathrm{H}, J=16.7 \mathrm{~Hz}), 4.70(\mathrm{~s}, 2 \mathrm{H}), 4.33$ $(\mathrm{s}, 2 \mathrm{H}), 3.86(\mathrm{~s}, 3 \mathrm{H}), 2.21(\mathrm{~s}, 3 \mathrm{H}) .{ }^{13} \mathrm{C}\left\{{ }^{1} \mathrm{H}\right\}$ NMR $\left(150 \mathrm{MHz}, \mathrm{CDCl}_{3}\right)$ $\delta(\mathrm{ppm}): 197.7(\mathrm{Cq}), 193.6(\mathrm{Cq}), 160.3(\mathrm{Cq}), 145.0(\mathrm{Cq}), 139.7(\mathrm{Cq})$, $137.9(\mathrm{CH}), 132.3(\mathrm{CH}), 131.6(\mathrm{CH}), 123.4(\mathrm{Cq}), 113.9(\mathrm{CH}), 72.6$ $\left(\mathrm{CH}_{2}\right), 65.7\left(\mathrm{CH}_{2}\right), 55.5\left(\mathrm{CH}_{3}\right), 27.7\left(\mathrm{CH}_{3}\right)$. HRMS (ESI) $\mathrm{m} / z$ : $[\mathrm{M}+$ $\mathrm{H}]^{+}$calcd for $\mathrm{C}_{16} \mathrm{H}_{17} \mathrm{O}_{4}{ }^{+} 273.1121$; found 273.1124 .

Methyl 4-(3-Oxo-5-(3-oxobut-1-en-1-yl)-3,6-dihydro- $2 \mathrm{H}$ pyran-4-yl)benzoate, $2 p$. Synthesized according to the general procedure GP5. Yellow solid, $45 \mathrm{mg}$, $75 \%$ yield, $E / Z$ ratio $40 / 60 . R_{f} 0.13$ (7/3 PE/EtOAc). (E)-2p ${ }^{1} \mathrm{H}$ NMR (600 MHz, $\left.\mathrm{CDCl}_{3}\right) \delta$ (ppm): 8.11 $(\mathrm{d}, 2 \mathrm{H}, J=8.1 \mathrm{~Hz}), 7.27(\mathrm{~d}, 2 \mathrm{H}, J=8.3 \mathrm{~Hz}), 7.07(\mathrm{~d}, 1 \mathrm{H}, J=16.6 \mathrm{~Hz})$, $6.35(\mathrm{~d}, 1 \mathrm{H}, J=16.7 \mathrm{~Hz}), 4.73(\mathrm{~s}, 2 \mathrm{H}), 4.35(\mathrm{~s}, 2 \mathrm{H}), 3.95(\mathrm{~s}, 3 \mathrm{H}), 2.19$ (s, 3H). ${ }^{13} \mathrm{C}\left\{{ }^{1} \mathrm{H}\right\}$ NMR $\left(150 \mathrm{MHz}, \mathrm{CDCl}_{3}\right) \delta(\mathrm{ppm}): 197.2(\mathrm{Cq})$, $192.7(\mathrm{Cq}), 166.7(\mathrm{Cq}), 147.3(\mathrm{Cq}), 139.2(\mathrm{Cq}), 136.6(\mathrm{CH}), 136.0$ (Cq), $132.5(\mathrm{CH}), 130.8(\mathrm{CH}), 130.7(\mathrm{Cq}), 129.6(\mathrm{CH}), 72.4\left(\mathrm{CH}_{2}\right)$, $65.5\left(\mathrm{CH}_{2}\right), 52.4\left(\mathrm{CH}_{3}\right), 27.8\left(\mathrm{CH}_{3}\right) .(\mathrm{Z})-2 \mathrm{p}{ }^{1} \mathrm{H}$ NMR $(600 \mathrm{MHz}$, $\left.\mathrm{CDCl}_{3}\right) \delta(\mathrm{ppm}): 8.02(\mathrm{~d}, 2 \mathrm{H}, J=8.2 \mathrm{~Hz}), 7.20(\mathrm{~d}, 2 \mathrm{H}, J=8.3 \mathrm{~Hz}), 6.25$ $(\mathrm{d}, 1 \mathrm{H}, J=12.3 \mathrm{~Hz}), 6.21(\mathrm{~d}, 1 \mathrm{H}, J=12.3 \mathrm{~Hz}), 4.56(\mathrm{~s}, 2 \mathrm{H}), 4.35(\mathrm{~s}$, $\left.2 \mathrm{H}), 3.92(\mathrm{~s}, 3 \mathrm{H}), 2.23(\mathrm{~s}, 3 \mathrm{H}) .{ }^{13} \mathrm{C}\left\{{ }^{1} \mathrm{H}\right\} \mathrm{NMR}\left(150 \mathrm{MHz}^{\mathrm{CDCl}}\right)_{3}\right) \delta$ $(\mathrm{ppm}): 198.3(\mathrm{Cq}), 193.0(\mathrm{Cq}), 166.8(\mathrm{Cq}), 153.2(\mathrm{Cq}), 137.3(\mathrm{Cq})$, $135.9(\mathrm{Cq}), 135.3(\mathrm{CH}), 131.2(\mathrm{CH}), 130.5(\mathrm{CH}), 130.2(\mathrm{Cq}), 129.3$ $(\mathrm{CH}), 72.8\left(\mathrm{CH}_{2}\right), 67.6\left(\mathrm{CH}_{2}\right), 52.4\left(\mathrm{CH}_{3}\right), 31.1\left(\mathrm{CH}_{3}\right)$. HRMS (ESI) $m / z:[\mathrm{M}+\mathrm{Na}]^{+}$calcd for $\mathrm{C}_{17} \mathrm{H}_{16} \mathrm{O}_{5} \mathrm{Na}^{+} 323.0890$; found 323.0873.

(E)-6-Ethyl-5-(3-oxobut-1-en-1-yl)-4-phenyl-2H-pyran$3(6 H)$-one, 2q. Synthesized according to the general procedure GP5.
Yellow oil, $33 \mathrm{mg}$, $62 \%$ yield. $R_{f} 0.12$ (9/1 PE/EtOAc). ${ }^{1} \mathrm{H}$ NMR (600 $\left.\mathrm{MHz}, \mathrm{CDCl}_{3}\right) \delta(\mathrm{ppm}): 7.34-7.27(\mathrm{~m}, 3 \mathrm{H}), 7.07-7.04(\mathrm{~m}, 2 \mathrm{H}), 6.29$ $(\mathrm{dd}, 1 \mathrm{H}, J=12.2,1.6 \mathrm{~Hz}), 6.10(\mathrm{~d}, 1 \mathrm{H}, J=12.2 \mathrm{~Hz}), 4.75-4.72(\mathrm{~m}$, $1 \mathrm{H}), 4.42(\mathrm{~d}, 1 \mathrm{H}, J=16.4 \mathrm{~Hz}), 4.32(\mathrm{dd}, 1 \mathrm{H}, J=16.4,1.3 \mathrm{~Hz}), 2.08(\mathrm{~s}$, $3 \mathrm{H}), 1.79-1.73(\mathrm{~m}, 2 \mathrm{H}), 1.03(\mathrm{t}, 3 \mathrm{H}, J=7.4 \mathrm{~Hz}) .{ }^{13} \mathrm{C}\left\{{ }^{1} \mathrm{H}\right\} \operatorname{NMR}(150$ $\left.\mathrm{MHz}, \mathrm{CDCl}_{3}\right) \delta(\mathrm{ppm}): 197.7(\mathrm{Cq}), 193.3(\mathrm{Cq}), 156.9(\mathrm{Cq}), 136.8$ $(\mathrm{CH}), 134.9(\mathrm{Cq}), 133.2(\mathrm{Cq}), 130.1(\mathrm{CH}), 129.6(\mathrm{CH}), 128.1(\mathrm{CH})$, $128.1(\mathrm{CH}), 77.2(\mathrm{CH}), 70.5\left(\mathrm{CH}_{2}\right), 30.9\left(\mathrm{CH}_{3}\right), 25.9\left(\mathrm{CH}_{2}\right), 10.1$ $\left(\mathrm{CH}_{3}\right)$. HRMS (ESI) $\mathrm{m} / z:[\mathrm{M}+\mathrm{H}]^{+}$calcd for $\mathrm{C}_{17} \mathrm{H}_{19} \mathrm{O}_{3}{ }^{+}$271.1329; found 271.1338 .

(E)-6-(tert-Butyl)-5-(3-oxobut-1-en-1-yl)-4-phenyl-2Hpyran-3(6H)-one, 2r. Synthesized according to the general procedure GP5. Brown oil, $28 \mathrm{mg}, 47 \%$ yield. $R_{f} 0.17$ (95/5 PE/EtOAc). ${ }^{1} \mathrm{H}$ NMR $\left(600 \mathrm{MHz}, \mathrm{CDCl}_{3}\right) \delta(\mathrm{ppm}): 7.34-7.27(\mathrm{~m}, 3 \mathrm{H}), 7.05-7.02(\mathrm{~m}, 2 \mathrm{H})$, $6.41(\mathrm{~d}, 1 \mathrm{H}, J=12.1 \mathrm{~Hz}), 6.00(\mathrm{~d}, 1 \mathrm{H}, J=12.1 \mathrm{~Hz}), 4.48(\mathrm{~d}, 1 \mathrm{H}, J=16.5$ $\mathrm{Hz}), 4.41(\mathrm{~s}, 1 \mathrm{H})$ superimposed to $4.39(\mathrm{~d}, 1 \mathrm{H}, J=16.7 \mathrm{~Hz}), 1.08(\mathrm{~s}$, 9H). ${ }^{13} \mathrm{C}\left\{{ }^{1} \mathrm{H}\right\}$ NMR $\left(150 \mathrm{MHz}, \mathrm{CDCl}_{3}\right) \delta(\mathrm{ppm}): 197.6(\mathrm{Cq}), 193.2$ $(\mathrm{Cq}), 139.3(\mathrm{Cq}), 135.6(\mathrm{Cq}), 133.9(\mathrm{Cq}), 129.7(\mathrm{CH}), 129.7(\mathrm{CH})$, $128.3(\mathrm{CH}), 128.3(\mathrm{CH}), 128.0(\mathrm{CH}), 84.2(\mathrm{CH}), 70.4\left(\mathrm{CH}_{2}\right), 37.6$ (Cq), $31.0\left(\mathrm{CH}_{3}\right), 28.2\left(\mathrm{CH}_{3}\right)$. HRMS (ESI) $\mathrm{m} / z:[\mathrm{M}+\mathrm{Na}]^{+}$calcd for $\mathrm{C}_{19} \mathrm{H}_{22} \mathrm{O}_{3} \mathrm{Na}^{+}$321.1461; found 321.1443 .

Gold(I)-Catalyzed Synthesis of (E)-4-(4-Benzoyl-1-tosyl-2,5dihydro-1H-pyrrol-3-yl)but-3-en-2-one, 3a. A solution of 1 a (1.0 equiv, $0.2 \mathrm{mmol}$ ) and 8 -methylquinoline $\mathrm{N}$-oxide (1.2 equiv) in DCE (0.1 M with respect to the furan-yne) was prepared, then [(IPr)Au$\left.\left(\mathrm{NTf}_{2}\right)\right]\left(0.05\right.$ equiv) was added, and the mixture was stirred at $80^{\circ} \mathrm{C}$ for $6 \mathrm{~h}$. Then, a few drops of $\mathrm{Et}_{3} \mathrm{~N}$ were added, and the solvent was evaporated under reduced pressure. The crude product was purified by flash column chromatography to afford pure dihydropyrrole $3 \mathbf{a}$ as the product. Yellow solid, $21 \mathrm{mg}, 27 \%$ yield, $\mathrm{mp} 139-140{ }^{\circ} \mathrm{C}\left(\mathrm{Et}_{2} \mathrm{O}\right) . R_{f}$ 0.24 (75/25 PE/EtOAc). ${ }^{1} \mathrm{H}$ NMR $\left(600 \mathrm{MHz} \mathrm{CDCl}_{3}\right) \delta(\mathrm{ppm})$ : 7.77-7.75 (m, 2H), 7.67-7.65 (m, 2H), 7.64-7.61 (m, 1H), 7.49$7.46(\mathrm{~m}, 2 \mathrm{H}), 7.37(\mathrm{~d}, 2 \mathrm{H}, J=8.1 \mathrm{~Hz}), 6.93(\mathrm{~d}, 1 \mathrm{H}, J=16.4 \mathrm{~Hz}), 6.01$ $(\mathrm{d}, 1 \mathrm{H}, J=16.4 \mathrm{~Hz}), 4.56(\mathrm{t}, 2 \mathrm{H}, J=4.1 \mathrm{~Hz}), 4.46(\mathrm{t}, 2 \mathrm{H}, J=4.1 \mathrm{~Hz})$, $2.46(\mathrm{~s}, 3 \mathrm{H}), 2.06(\mathrm{~s}, 3 \mathrm{H}) \cdot{ }^{13} \mathrm{C}\left\{{ }^{1} \mathrm{H}\right\} \mathrm{NMR}\left(150 \mathrm{MHz}, \mathrm{CDCl}_{3}\right) \delta(\mathrm{ppm})$ : $197.7(\mathrm{Cq}), 191.8(\mathrm{Cq}), 144.4(\mathrm{Cq}), 140.3(\mathrm{Cq}), 138.5(\mathrm{Cq}), 137.2$ (Cq), $134.3(\mathrm{CH}), 133.3(\mathrm{Cq}), 132.3(\mathrm{CH}), 132.2(\mathrm{CH}), 130.3(\mathrm{CH})$, $129.3(\mathrm{CH}), 129.1(\mathrm{CH}), 127.7(\mathrm{CH}), 57.5\left(\mathrm{CH}_{2}\right), 55.2\left(\mathrm{CH}_{2}\right), 27.3$ $\left(\mathrm{CH}_{3}\right), 21.7\left(\mathrm{CH}_{3}\right)$. HRMS (ESI) $\mathrm{m} / z:[\mathrm{M}+\mathrm{Na}]^{+}$calcd for $\mathrm{C}_{22} \mathrm{H}_{21} \mathrm{NO}_{4} \mathrm{SNa}^{+}$418.1083; found 418.1057 .

Gold(I)-Catalyzed Synthesis of (E)-4-Methyl- $N$-((5-methylfuran-2-yl)methyl)-N-(3-oxo-3-phenylprop-1-en-1-yl)benzenesulfonamide, $4 \mathrm{a}$. A solution of $1 \mathrm{a}$ (1.0 equiv, $0.2 \mathrm{mmol}$ ) and 8-methylquinoline $\mathrm{N}$-oxide ( 1.2 equiv) in DCE ( $0.1 \mathrm{M}$ with respect to the furan-yne $)$ was prepared, then [(MorDalPhos) $\mathrm{Au}(\mathrm{NCMe})] \mathrm{SbF}_{6}$ ( 0.05 equiv) was added, and the mixture was stirred at room temperature for $20 \mathrm{~h}$. Then, a few drops of $\mathrm{Et}_{3} \mathrm{~N}$ were added, and the solvent was evaporated under reduced pressure. The crude product was purified by flash column chromatography to afford pure vinyl ketone $4 \mathbf{a}$ as the product. White solid, $59 \mathrm{mg}, 74 \%$ yield, $\mathrm{mp} 127-128{ }^{\circ} \mathrm{C}$ (EtOAc). $R_{f} 0.20$ (9/1 PE/EtOAc). ${ }^{1} \mathrm{H}$ NMR $\left(600 \mathrm{MHz}, \mathrm{CDCl}_{3}\right) \delta$ (ppm): $8.26(\mathrm{~d}, 1 \mathrm{H}, J=13.6 \mathrm{~Hz}), 7.88-7.85(\mathrm{~m}, 2 \mathrm{H}), 7.71(\mathrm{~d}, 2 \mathrm{H}, J=$ $8.4 \mathrm{~Hz}), 7.54-7.51(\mathrm{~m}, 1 \mathrm{H}), 7.46-7.42(\mathrm{~m}, 2 \mathrm{H}), 7.29(\mathrm{~d}, 2 \mathrm{H}, J=8.0$ $\mathrm{Hz}), 6.50(\mathrm{~d}, 1 \mathrm{H}, J=13.6 \mathrm{~Hz}), 6.07(\mathrm{~d}, 1 \mathrm{H}, J=3.1 \mathrm{~Hz}), 5.85-5.84(\mathrm{~m}$, $1 \mathrm{H}), 4.74(\mathrm{~s}, 2 \mathrm{H}), 2.42(\mathrm{~s}, 3 \mathrm{H}), 2.07(\mathrm{~d}, 3 \mathrm{H}, J=1.1 \mathrm{~Hz}) .{ }^{13} \mathrm{C}\left\{{ }^{1} \mathrm{H}\right\} \mathrm{NMR}$ $\left(150 \mathrm{MHz}, \mathrm{CDCl}_{3}\right) \delta(\mathrm{ppm}): 189.4(\mathrm{Cq}), 152.4(\mathrm{Cq}), 146.0(\mathrm{Cq})$, $144.9(\mathrm{Cq}), 142.6(\mathrm{CH}), 138.7(\mathrm{Cq}), 135.7(\mathrm{Cq}), 132.5(\mathrm{CH}), 130.1$ $(\mathrm{CH}), 128.6(\mathrm{CH}), 128.2(\mathrm{CH}), 127.6(\mathrm{CH}), 110.7(\mathrm{CH}), 106.7$ $(\mathrm{CH}), 104.0(\mathrm{CH}), 43.3\left(\mathrm{CH}_{2}\right), 21.7\left(\mathrm{CH}_{3}\right), 13.5\left(\mathrm{CH}_{3}\right)$. HRMS (ESI) $\mathrm{m} / z:[\mathrm{M}+\mathrm{H}]^{+}$calcd for $\mathrm{C}_{22} \mathrm{H}_{22} \mathrm{NO}_{4} \mathrm{~S}^{+}$396.1264; found 396.1263.

Synthesis of 5-(3-Oxo-1-(phenylthio)butyl)-4-phenyl-1tosyl-1,6-dihydropyridin-3(2H)-one, 5. A reported procedure was followed. ${ }^{15}$ To a $0.1 \mathrm{M}$ solution of $2 \mathrm{a}$ (1.0 equiv, $0.1 \mathrm{mmol}$ ) in DCM, at $0{ }^{\circ} \mathrm{C}$ under air, thiophenol (1.2 equiv) and $\mathrm{Et}_{3} \mathrm{~N}$ (0.1 equiv) were added. The mixture was allowed to warm to room temperature and was stirred for $2 \mathrm{~h}$. Then, the volatiles were removed under reduced pressure. The crude product was purified by flash column chromatography to obtain pure sulfide $\mathbf{5}$ as the product. Two diastereoisomers, arising from the chiral carbon and atropisomerism, could be separated. Colorless oil, $34 \mathrm{mg}, 68 \%$ overall yield. 5-D1 $R_{f} 0.15$ 
(8/2 PE/EtOAc). ${ }^{1} \mathrm{H}$ NMR (600 MHz, $\left.\mathrm{CDCl}_{3}\right) \delta$ (ppm): 7.70-7.68 $(\mathrm{m}, 2 \mathrm{H}), 7.34(\mathrm{~d}, 2 \mathrm{H}, J=7.9 \mathrm{~Hz}), 7.32-7.30(\mathrm{~m}, 3 \mathrm{H}), 7.29-7.26(\mathrm{~m}$, $1 \mathrm{H}), 7.25-7.22(\mathrm{~m}, 2 \mathrm{H}), 7.12-7.10(\mathrm{~m}, 2 \mathrm{H}), 6.81-6.78(\mathrm{~m}, 2 \mathrm{H}), 4.17$ $(\mathrm{dd}, 1 \mathrm{H}, J=18.4,1.6 \mathrm{~Hz}), 4.06(\mathrm{dd}, 1 \mathrm{H}, J=18.4,1.6 \mathrm{~Hz}), 3.98(\mathrm{dd}, 1 \mathrm{H}$, $J=16.7,1.6 \mathrm{~Hz}), 3.79(\mathrm{dd}, 1 \mathrm{H}, J=16.7,1.7 \mathrm{~Hz}), 3.38(\mathrm{t}, 1 \mathrm{H}, J=7.7$ $\mathrm{Hz}), 2.65(\mathrm{dd}, 1 \mathrm{H}, J=14.8,7.4 \mathrm{~Hz}), 2.59(\mathrm{dd}, 1 \mathrm{H}, J=14.9,8.1 \mathrm{~Hz})$, $2.45(\mathrm{~s}, 3 \mathrm{H}), 2.07(\mathrm{~s}, 3 \mathrm{H}) .{ }^{13} \mathrm{C}\left\{{ }^{1} \mathrm{H}\right\} \mathrm{NMR}\left(150 \mathrm{MHz}, \mathrm{CDCl}_{3}\right) \delta(\mathrm{ppm})$ : $203.3(\mathrm{Cq}), 190.1(\mathrm{Cq}), 152.6(\mathrm{Cq}), 144.6(\mathrm{Cq}), 138.0(\mathrm{Cq}), 133.4$ $(\mathrm{Cq}), 132.9(\mathrm{CH}), 132.7(\mathrm{Cq}), 131.8(\mathrm{Cq}), 130.3(\mathrm{CH}), 129.5(\mathrm{CH})$, $129.5(\mathrm{CH}), 128.8(\mathrm{CH}), 128.7(\mathrm{CH}), 128.4(\mathrm{CH}), 127.8(\mathrm{CH}), 54.5$ $(\mathrm{CH}), 52.8\left(\mathrm{CH}_{2}\right), 49.2\left(\mathrm{CH}_{2}\right), 34.1\left(\mathrm{CH}_{2}\right), 27.6\left(\mathrm{CH}_{3}\right), 21.7\left(\mathrm{CH}_{3}\right)$. HRMS (ESI) $m / z$ : $[\mathrm{M}+\mathrm{Na}]^{+}$calcd for $\mathrm{C}_{28} \mathrm{H}_{27} \mathrm{NO}_{4} \mathrm{~S}_{2} \mathrm{Na}^{+}$528.1274, found 528.1265. 5-D2 $R_{f} 0.08$ (8/2 PE/EtOAc). ${ }^{1} \mathrm{H}$ NMR $(600 \mathrm{MHz}$, $\left.\mathrm{CDCl}_{3}\right) \delta(\mathrm{ppm}): 7.75(\mathrm{~d}, 2 \mathrm{H}, J=8.2 \mathrm{~Hz}), 7.40-7.35(\mathrm{~m}, 3 \mathrm{H}), 7.33-$ $7.30(\mathrm{~m}, 1 \mathrm{H}), 7.26-7.21(\mathrm{~m}, 6 \mathrm{H}), 7.20-7.14(\mathrm{~m}, 2 \mathrm{H}), 4.56(\mathrm{dd}, 1 \mathrm{H}, J$ $=17.1,1.6 \mathrm{~Hz}), 4.32(\mathrm{t}, 1 \mathrm{H}, J=7.6 \mathrm{~Hz}), 3.93(\mathrm{dd}, 1 \mathrm{H}, J=17.0,1.7 \mathrm{~Hz})$ superimposed to $3.92(\mathrm{dd}, 1 \mathrm{H}, J=16.8,1.6 \mathrm{~Hz}), 3.79(\mathrm{dd}, 1 \mathrm{H}, J=16.7$, $1.7 \mathrm{~Hz}), 2.86(\mathrm{dd}, 1 \mathrm{H}, J=17.1,7.5 \mathrm{~Hz}), 2.65(\mathrm{dd}, 1 \mathrm{H}, J=17.1,7.5 \mathrm{~Hz})$, $2.46(\mathrm{~s}, 3 \mathrm{H}), 2.10(\mathrm{~s}, 3 \mathrm{H}) .{ }^{13} \mathrm{C}\left\{{ }^{1} \mathrm{H}\right\} \mathrm{NMR}\left(150 \mathrm{MHz}, \mathrm{CDCl}_{3}\right) \delta(\mathrm{ppm})$ : $203.8(\mathrm{Cq}), 190.7(\mathrm{Cq}), 152.2(\mathrm{Cq}), 144.7(\mathrm{Cq}), 137.3(\mathrm{Cq}), 134.6$ $(\mathrm{CH}), 133.0(\mathrm{Cq}), 131.9(\mathrm{Cq}), 131.8(\mathrm{Cq}), 130.3(\mathrm{CH}), 129.6(\mathrm{CH})$, $129.5(\mathrm{CH}), 129.1(\mathrm{CH}), 128.3(\mathrm{CH}), 128.2(\mathrm{CH}), 128.1(\mathrm{CH}), 128.1$ $(\mathrm{CH}), 53.4\left(\mathrm{CH}_{2}\right), 46.7(\mathrm{CH}), 45.7\left(\mathrm{CH}_{2}\right), 44.9\left(\mathrm{CH}_{2}\right), 30.4\left(\mathrm{CH}_{3}\right)$, $21.7\left(\mathrm{CH}_{3}\right)$. HRMS (ESI) $m / z:[\mathrm{M}+\mathrm{Na}]^{+}$calcd for $\mathrm{C}_{28} \mathrm{H}_{27} \mathrm{NO}_{4} \mathrm{~S}_{2} \mathrm{Na}^{+}$ 528.1274; found 528.1265.

Synthesis of 5-(3-Oxobutyl)-4-phenyl-1-tosyl-1,6-dihydropyridin-3(2H)-one, 6 . To $0.1 \mathrm{M}$ solution of $2 \mathrm{a}$ (1.0 equiv, 0.1 $\mathrm{mmol})$ in EtOAc, Pd/C $(10 \% \mathrm{w} / \mathrm{w})$ was added. The flask was evacuated and backfilled with $\mathrm{H}_{2}$ three times, and then the mixture was stirred at $50{ }^{\circ} \mathrm{C}$ under a $\mathrm{H}_{2}$ atmosphere for $2 \mathrm{~h}$. After that time, the mixture was cooled down to room temperature and filtered over a Celite pad, and the volatiles were removed under reduced pressure. The crude product was purified by flash column chromatography to afford the partially hydrogenated product 6 . Colorless oil, $28 \mathrm{mg}, 71 \%$ yield. $R_{f}$ 0.22 (65/35 PE/EtOAc). ${ }^{1} \mathrm{H}$ NMR (600 $\left.\mathrm{MHz}, \mathrm{CDCl}_{3}\right) \delta(\mathrm{ppm})$ : 7.72-7.69 (m, 2H), 7.41-7.36 (m, 2H), 7.34-7.29 (m, 3H), 6.84$6.82(\mathrm{~m}, 2 \mathrm{H}), 4.05(\mathrm{~s}, 2 \mathrm{H}), 3.91(\mathrm{~s}, 2 \mathrm{H}), 2.46(\mathrm{~s}, 3 \mathrm{H}), 2.43(\mathrm{t}, 2 \mathrm{H}, J=$ $7.7 \mathrm{~Hz}), 2.33(\mathrm{t}, 2 \mathrm{H}, J=7.6 \mathrm{~Hz}), 2.05(\mathrm{~s}, 3 \mathrm{H}) .{ }^{13} \mathrm{C}\left\{{ }^{1} \mathrm{H}\right\} \operatorname{NMR}(150$ $\left.\mathrm{MHz}, \mathrm{CDCl}_{3}\right) \delta(\mathrm{ppm}): 206.2(\mathrm{Cq}), 190.2(\mathrm{Cq}), 154.7$ (Cq), 144.7 $(\mathrm{Cq}), 137.3(\mathrm{Cq}), 133.1(\mathrm{Cq}), 132.9(\mathrm{Cq}), 130.3(\mathrm{CH}), 129.4(\mathrm{CH})$, $128.6(\mathrm{CH}), 128.2(\mathrm{CH}), 127.9(\mathrm{CH}), 52.9\left(\mathrm{CH}_{2}\right), 48.1\left(\mathrm{CH}_{2}\right), 40.9$ $\left(\mathrm{CH}_{2}\right), 29.8\left(\mathrm{CH}_{3}\right), 26.9\left(\mathrm{CH}_{2}\right), 21.7\left(\mathrm{CH}_{3}\right)$. HRMS (ESI) $\mathrm{m} / z:[\mathrm{M}+$ $\mathrm{H}]^{+}$calcd for $\mathrm{C}_{22} \mathrm{H}_{24} \mathrm{NO}_{4} \mathrm{~S}^{+} 398.1421$; found 398.1418.

Synthesis of $(E)-5-(3-H y d r o x y b u t-1-e n-1-y l)-4-p h e n y l-1-$ tosyl-1,2,3,6-tetrahydropyridin-3-ol, 7. To $0.1 \mathrm{M}$ solution of 2a ( 1.0 equiv, $0.1 \mathrm{mmol}$ ) in $\mathrm{DCM} / \mathrm{MeOH} 1 / 1$ at $0{ }^{\circ} \mathrm{C}, \mathrm{NaBH}_{4}$ (1.0 equiv) was added, and the mixture was allowed to warm to room temperature and stirred for $1.5 \mathrm{~h}$. Then, the solvent was evaporated under reduced pressure to a quarter of the initial volume. Water was added, and the mixture was extracted three times with DCM; the combined organic layers were dried over anhydrous $\mathrm{Na}_{2} \mathrm{SO}_{4}$ and filtered, and the volatiles were removed under reduced pressure. The crude product was purified by flash column chromatography to afford the diol product 7 as a $1 / 1$ mixture of diastereoisomers. Colorless oil, $21 \mathrm{mg}, 53 \%$ yield. $R_{f} 0.24$ (6/ $4 \mathrm{PE} / \mathrm{EtOAc}) .{ }^{1} \mathrm{H}$ NMR $\left(600 \mathrm{MHz}, \mathrm{CDCl}_{3}\right) \delta(\mathrm{ppm}): 7.76(\mathrm{~d}, 2 \mathrm{H}, J=$ $8.2 \mathrm{~Hz})$ superimposed to $7.76(\mathrm{~d}, 2 \mathrm{H}, J=8.3 \mathrm{~Hz}), 7.40-7.37(\mathrm{~m}, 4 \mathrm{H})$, $7.26-7.34(\mathrm{~m}, 4 \mathrm{H}), 7.33-7.30(\mathrm{~m}, 2 \mathrm{H}), 7.17-7.14(\mathrm{~m}, 4 \mathrm{H}), 6.12$ (d, $2 \mathrm{H}, J=16.2 \mathrm{~Hz}), 5.71(\mathrm{dd}, 1 \mathrm{H}, J=16.2,6.4 \mathrm{~Hz})$ superimposed to 5.71 $(\mathrm{dd}, 1 \mathrm{H}, J=16.2,6.7 \mathrm{~Hz}), 4.42-4.38(\mathrm{~m}, 2 \mathrm{H}), 4.25-4.17(\mathrm{~m}, 4 \mathrm{H})$, $3.74(\mathrm{dd}, 1 \mathrm{H}, J=11.8,3.4 \mathrm{~Hz}), 3.68(\mathrm{dd}, 1 \mathrm{H}, J=11.8,3.6 \mathrm{~Hz}), 3.44(\mathrm{~d}$, $1 \mathrm{H}, J=15.6 \mathrm{~Hz}), 3.40(\mathrm{~d}, 1 \mathrm{H}, J=15.6 \mathrm{~Hz}), 3.02(\mathrm{dd}, 1 \mathrm{H}, J=11.9,3.3$ $\mathrm{Hz}), 2.95(\mathrm{dd}, 1 \mathrm{H}, J=11.8,3.2 \mathrm{~Hz}), 2.46(\mathrm{~s}, 6 \mathrm{H}), 1.23(\mathrm{~d}, 3 \mathrm{H}, J=6.4$ $\mathrm{Hz}), 1.20(\mathrm{~d}, 3 \mathrm{H}, J=6.3 \mathrm{~Hz}) \cdot{ }^{13} \mathrm{C}\left\{{ }^{1} \mathrm{H}\right\} \mathrm{NMR}\left(150 \mathrm{MHz}, \mathrm{CDCl}_{3}\right) \delta$ $(\mathrm{ppm}): 144.3(\mathrm{Cq}), 144.2(\mathrm{Cq}), 138.4(\mathrm{Cq}), 138.4(\mathrm{Cq}), 138.2(\mathrm{Cq})$, $138.1(\mathrm{Cq}), 134.7(\mathrm{CH}), 134.6(\mathrm{CH}), 132.9(\mathrm{Cq}), 132.8(\mathrm{Cq}), 130.1$ $(\mathrm{CH}), 129.3(\mathrm{CH}), 129.3(\mathrm{CH}), 128.8(\mathrm{Cq}), 128.8(\mathrm{Cq}), 128.6(\mathrm{CH})$, $128.0(\mathrm{CH}), 128.0(\mathrm{CH}), 126.2(\mathrm{CH}), 125.9(\mathrm{CH}), 69.1(\mathrm{CH}), 69.0$ $(\mathrm{CH}), 67.8(\mathrm{CH}), 67.8(\mathrm{CH}), 50.7\left(\mathrm{CH}_{2}\right), 50.6\left(\mathrm{CH}_{2}\right), 45.5\left(\mathrm{CH}_{2}\right)$, $45.5\left(\mathrm{CH}_{2}\right), 23.5\left(\mathrm{CH}_{3}\right), 23.5\left(\mathrm{CH}_{3}\right), 21.7\left(\mathrm{CH}_{3}\right)$. HRMS (ESI) $\mathrm{m} / \mathrm{z}$ : $[\mathrm{M}+\mathrm{Na}]^{+}$calcd for $\mathrm{C}_{22} \mathrm{H}_{25} \mathrm{NO}_{4} \mathrm{SNa}^{+}$422.4942; found 422.4946 .

\section{ASSOCIATED CONTENT}

\section{Supporting Information}

The Supporting Information is available free of charge at https://pubs.acs.org/doi/10.1021/acs.joc.1c00746.

Complete list of optimization experiments, reaction schemes for the synthesis of substrates, crystallographic details, computational details, NMR spectra of new compounds (PDF)

FAIR data, including the primary NMR FID files, for compounds $1 \mathrm{a}-\mathrm{s}, 2 \mathrm{a}-\mathrm{r}$, int-7, int-8, int-9, int-12, 5, 6, 7 (ZIP)

\section{Accession Codes}

CCDC 2067578-2067580 contain the supplementary crystallographic data for this paper. These data can be obtained free of charge via www.ccdc.cam.ac.uk/data_request/cif, or by emailing data_request@ccdc.cam.ac.uk, or by contacting The Cambridge Crystallographic Data Centre, 12 Union Road, Cambridge CB2 1EZ, UK; fax: +44 1223336033.

\section{AUTHOR INFORMATION}

\section{Corresponding Authors}

Cristina Prandi - Dipartimento di Chimica, Università degli Studi di Torino, I-10125 Torino, Italy; ○ orcid.org/00000001-9510-8783; Email: cristina.prandi@unito.it

Stefano Nejrotti - Dipartimento di Chimica, Università degli Studi di Torino, I-10125 Torino, Italy; ㅇo이.org/0000-

0002-6017-5705; Email: stefano.nejrotti@unito.it

\section{Authors}

Francesco Marra - Dipartimento di Chimica, Università degli Studi di Torino, I-10125 Torino, Italy

Emanuele Priola - Dipartimento di Chimica, Università degli Studi di Torino, I-10125 Torino, Italy; 이잉.org/00000002-0270-738X

Andrea Maranzana - Dipartimento di Chimica, Università degli Studi di Torino, I-10125 Torino, Italy

Complete contact information is available at:

https://pubs.acs.org/10.1021/acs.joc.1c00746

\section{Author Contributions}

Cristina Prandi: conceptualization, writing, funding acquisition, supervision. Stefano Nejrotti: conceptualization, investigation, data analysis, writing. Francesco Marra, Emanuele Priola: investigation, data analysis. Andrea Maranzana: investigation, data analysis, writing. All authors have given approval to the final version of the manuscript.

Notes

The authors declare no competing financial interest.

\section{ACKNOWLEDGMENTS}

The Italian Ministry of Research, Regione Piemonte, Cassa di Risparmio di Torino and Huvepharma srl are acknowledged for funding.

\section{REFERENCES}

(1) (a) Hashmi, A. S. K. Homogeneous Gold Catalysts and Alkynes: A Successful Liaison. Gold Bull. 2003, 36, 3-9. (b) Hashmi, A. S. K. GoldCatalyzed Organic Reactions. Chem. Rev. 2007, 107, 3180-3211. (c) Li, Z.; Brouwer, C.; He, C. Gold-Catalyzed Organic Transformations. Chem. Rev. 2008, 108, 3239-3265. (d) Dorel, R.; Echavarren, A. M. Gold(I)-Catalyzed Activation of Alkynes for the 
Construction of Molecular Complexity. Chem. Rev. 2015, 115, 90289072.

(2) (a) First report: Hashmi, A. S. K.; Schwarz, L.; Choi, J.-H.; Frost, T. M. A New Gold-Catalyzed C-C Bond Formation. Angew. Chem., Int. Ed. 2000, 39, 2285-2288. (b) Kirillova, M. S.; Miloserdov, F. M.; Echavarren, A. M. Hydroarylation of Alkynes using $\mathrm{Cu}, \mathrm{Ag}$, and $\mathrm{Au}$ Catalysts. Catalytic Hydroarylation of Carbon-Carbon Multiple Bonds 2017, 217-303. (c) Muratore, M. E.; Echavarren, A. M. Gold-catalyzed hydroarylation of alkynes. PATAI'S Chemistry of Functional Groups 2015, 1-96. (d) Nejrotti, S.; Prandi, C. Gold Catalysis and Furans: A Powerful Match for Synthetic Connections. Synthesis 2021, 53, 10461060.

(3) (a) Hashmi, A. S. K.; Frost, T. M.; Bats, J. Highly Selective GoldCatalyzed Arene Synthesis. J. Am. Chem. Soc. 2000, 122, 11553-11554. (b) Hashmi, A. S. K.; Grundl, L. Gold Catalysis: Five New Bonds by a Domino Hydroarylation/Cycloisomerization. Tetrahedron 2005, 61, 6231-6236. (c) Hashmi, A. S. K.; Weyrauch, J. P.; Kurpejović, E.; Frost, T. M.; Miehlich, B.; Frey, W.; Bats, J. W. Gold Catalysis: Phenol Synthesis in the Presence of Functional Groups. Chem. - Eur. J. 2006, 12, 5806-5814. (d) Hashmi, A. S. K.; Salathé, R.; Frey, W. Gold Catalysis: No Steric Limitations in the Phenol Synthesis. Chem. - Eur. J. 2006, 12, 6991-6996. (e) Hashmi, A. S. K.; Kurpejović, E.; Wölfle, M.; Frey, W.; Bats, J. W. Gold Catalysis: Oxepines from $\gamma$-Alkynylfurans. Adv. Synth. Catal. 2007, 349, 1743-1750. (f) Hashmi, A. S. K.; Rudolph, M.; Siehl, H.-U.; Tanaka, M.; Bats, J. W.; Frey, W. Gold Catalysis: Deuterated Substrates as the Key for an Experimental Insight into the Mechanism and Selectivity of the Phenol Synthesis. Chem. Eur. J. 2008, 14, 3703-3708. (g) Hashmi, A. S. K.; Rudolph, M.; Huck, J.; Frey, W.; Bats, J. W.; Hamzić, M. Gold Catalysis: Switching the Pathway of the Furan-Yne Cyclization. Angew. Chem., Int. Ed. 2009, 48, 5848-5852. (h) Rudolph, M.; McCreery, M. Q.; Frey, W.; Hashmi, A. S. K. High Chemoselectivity in the Phenol Synthesis. Beilstein. Beilstein J. Org. Chem. 2011, 7, 794-801. (i) Hashmi, A. S. K.; Häffner, T.; Rudolph, M.; Rominger, F. Gold Catalysis: Domino Reaction of EnDiynes to Highly Substituted Phenols. Chem. - Eur. J. 2011, 17, 81958201. (j) Zeiler, A.; Ziegler, M. J.; Rudolph, M.; Rominger, F.; Hashmi, A. S. K. Scope and Limitations of the Intermolecular Furan-Yne Cyclization. Adv. Synth. Catal. 2015, 357, 1507-1514.

(4) Leboeuf, D.; Gaydou, M.; Wang, Y.; Echavarren, A. M. Intermolecular reactions of gold(I)-carbenes with furans by related mechanisms. Org. Chem. Front. 2014, 1, 759-764.

(5) Yang, J. M.; Tang, X. Y.; Shi, M. Gold(I)-Catalyzed Intramolecular Cycloisomerization of Propargylic Esters with Furan Rings. Chem. - Eur. J. 2015, 21, 4534-4540.

(6) $\alpha$-Oxo gold carbenes are in equilibrium with the corresponding carbenoid species, generated by attack of the pyridine onto the carbene center. (a) Schulz, J.; Jasikova, L.; Skriba, A.; Roithova, J. Role of Gold(I) $\alpha$-Oxo Carbenes in the Oxidation Reactions of Alkynes Catalyzed by Gold(I) Complexes. J. Am. Chem. Soc. 2014, 136, 1151311523. (b) Schulz, J.; Jašík, J.; Gray, A.; Roithová, J. Formation of Oxazoles from Elusive Gold(I) $\alpha$-Oxocarbenes: A Mechanistic Study. Chem. - Eur. J. 2016, 22, 9827-9834.

(7) Seminal reports: (a) Li, G.; Zhang, L. Gold-Catalyzed Intramolecular Redox Reaction of Sulfinyl Alkynes: Efficient Generation of a-Oxo Gold Carbenoids and Application in Insertion into R-CO Bonds. Angew. Chem., Int. Ed. 2007, 46, 5156-5159. (b) Shapiro, N. D.; Toste, F. D. Rearrangement of Alkynyl Sulfoxides Catalyzed by Gold(I) Complexes. J. Am. Chem. Soc. 2007, 129, 4160-4161. Reviews on the topic: (c) Yeom, H.-S.; Shin, S. Catalytic Access to $\alpha$-Oxo Gold Carbenes by N-O Bond Oxidants. Acc. Chem. Res. 2014, 47, 966-977. (d) Zhang, L. A Non-Diazo Approach to $\alpha$-Oxo Gold Carbenes via Gold-Catalyzed Alkyne Oxidation. Acc. Chem. Res. 2014, 47, 877-888. (e) Bhunia, S.; Ghosh, P.; Patra, S. R. Gold-Catalyzed Oxidative Alkyne Functionalization by $\mathrm{N}-\mathrm{O} / \mathrm{S}-\mathrm{O} / \mathrm{C}-\mathrm{O}$ Bond Oxidants. Adv. Synth. Catal. 2020, 362, 3664-3708. For a recent systematic study on this chemistry, see: (f) Schießl, J.; Stein, P. M.; Stirn, J.; Emler, K.; Rudolph, M.; Rominger, F.; Hashmi, A. S. K. Strategic Approach on N-Oxides in Gold Catalysis - A Case Study. Adv. Synth. Catal. 2019, 361, 725-738.
(8) (a) Oppedisano, A.; Prandi, C.; Venturello, P.; Deagostino, A.; Goti, G.; Scarpi, D.; Occhiato, E. G. Synthesis of Vinylogous Amides by Gold(I)-Catalyzed Cyclization of N-Boc-Protected 6-Alkynyl-3,4dihydro-2H-pyridines. J. Org. Chem. 2013, 78, 11007-11016. (b) Scarpi, D.; Begliomini, S.; Prandi, C.; Oppedisano, A.; Deagostino, A.; Gómez-Bengoa, E.; Fiser, B.; Occhiato, E. G. GoldCatalysed Synthesis of Exocyclic Vinylogous Amides and $\beta$-Amino Ketones: A Detailed Study on the 5-exo/6-endo-dig Selectivity, Methodology and Scope. Eur. J. Org. Chem. 2015, 2015, 3251-3265. (c) Nejrotti, S.; Prina Cerai, G.; Oppedisano, A.; Maranzana, A.; Occhiato, E. G.; Scarpi, D.; Deagostino, A.; Prandi, C. A Gold(I)Catalyzed Oxidative Rearrangement of Heterocycle-Derived 1,3Enynes Provides an Efficient and Selective Route to Divinyl Ketones. Eur. J. Org. Chem. 2017, 2017, 6228-6238. (d) Nejrotti, S.; Ghinato, S.; Gini, E. C.; Scarpi, D.; Occhiato, E. G.; Maranzana, A.; Prandi, C. Gold(I)-Catalysed Hydroarylation of Lactam-Derived Enynes as an Entry to Tetrahydrobenzo $[g]$ quinolines. Eur. J. Org. Chem. 2020, 2020, 646-653.

(9) The "classical" furan-yne with a terminal alkyne (int-6 in the SI) only underwent the Hashmi's phenol synthesis, and oxidative reaction was not observed under the tested reaction conditions.

(10) CCDC codes 2067578-2067580 contain the supplementary crystallographic data for $2 \mathrm{a}, 3 \mathrm{a}$, and $4 \mathrm{a}$.

(11) (a) Ito, M.; Kawasaki, R.; Kanyiva, K. S.; Shibata, T. Catalytic Dearomative Spirocyclization via Gold Carbene Species Derived from Ynamides: Efficient Synthesis of 2-Azaspiro[4.5]decan-3-ones. Chem. Eur. J. 2018, 24, 3721-3724 see also:. (b) Hashmi, A. S. K. Homogeneous Gold Catalysis Beyond Assumptions and ProposalsCharacterized Intermediates. Angew. Chem., Int. Ed. 2010, 49, 52325241. (c) Lauterbach, T.; Asiri, A. M.; Hashmi, A. S. K. Organometallic Intermediates of Gold Catalysis. Adv. Organomet. Chem. 2014, 62, 261-297.

(12) Hashmi, A. S. K.; Wölfle, M.; Ata, F.; Hamzic, M.; Salathé, R.; Frey, W. Gold Catalysis: Dihydroisobenzofurans and Isochromanes by theIntramolecular Furan/Alkyne Reaction. Adv. Synth. Catal. 2006, $348,2501-2508$

(13) (a) Lu, B.; Li, C.; Zhang, L. Gold-Catalyzed Highly Regioselective Oxidation of $\mathrm{C}-\mathrm{C}$ Triple Bonds without Acid Additives: Propargyl Moieties as Masked $\alpha, \beta$-Unsaturated Carbonyls. J. Am. Chem. Soc. 2010, 132, 14070-14072. (b) Davies, P. W.; Cremonesi, A.; Martin, N. Site-Specific Introduction of Gold-Carbenoids by Intermolecular Oxidation of Ynamides or Ynol Ethers. Chem. Commun. 2011, 47, 379-381. (c) Ji, K.; D’souza, B.; Nelson, J.; Zhang, L. GoldCatalyzed Oxidation of Propargylic Ethers with Internal C-C Triple Bonds: Impressive Regioselectivity Enabled by Inductive Effect. $J$. Organomet. Chem. 2014, 770, 142-145.

(14) Ji, K.; Zheng, Z.; Wang, Z.; Zhang, L. Enantioselective Oxidative Gold Catalysis Enabled by a Designed Chiral P,N-Bidentate Ligand. Angew. Chem., Int. Ed. 2015, 54, 1245-1249.

(15) Jouha, J.; Buttard, F.; Lorion, M.; Berthonneau, C.; Khouili, M.; Hiebel, M.-A.; Guillaumet, G.; Brière, J.-F. o.; Suzenet, F. Domino AzaMichael-ih-Diels-Alder Reaction to Various 3-Vinyl-1,2,4-triazines: Access to Polysubstituted Tetrahydro-1,6-naphthyridines. Org. Lett. 2017, 19, 4770-4773.

(16) Zhao, Y.; Truhlar, D. G. The M06 Suite of Density Functionals for Main Group Thermochemistry, Thermochemical Kinetics, Noncovalent Interactions, Excited States, and Transition Elements: Two New Functionals and Systematic Testing of Four M06-Class Functionals and 12 Other Functionals. Theor. Chem. Acc. 2008, 120, 215241.

(17) Dapprich, S.; Komáromi, I.; Byun, K. S.; Morokuma, K.; Frisch, M. J. A New ONIOM Implementation in Gaussian 98. 1. The Calculation of Energies, Gradients and Vibrational Frequencies and Electric Field Derivatives. J. Mol. Struct.: THEOCHEM 1999, 462, 121.

(18) Weigend, F. Accurate Coulomb-Fitting Basis Sets for H to Rn. Phys. Chem. Chem. Phys. 2006, 8, 1057-1065.

(19) Collins, J. B.; von R. Schleyer, P.; Binkley, J. S.; Pople, J. A. SelfConsistent Molecular Orbital Methods. XVII. Geometries and Binding 
Energies of Second-Row Molecules. A Comparison of Three Basis Sets.

J. Chem. Phys. 1976, 64, 5142-5151.

(20) Marenich, A. V.; Cramer, C. J.; Truhlar, D. G. Universal Solvation Model Based on Solute Electron Density and a Continuum Model of the Solvent Defined by the Bulk Dielectric Constant and Atomic Surface Tensions. J. Phys. Chem. B 2009, 113, 6378-6396.

(21) Lu, T.; Chen, F. Multiwfn: a Multifunctional Wavefunction Analyzer. J. Comput. Chem. 2012, 33, 580-592.

(22) Johnson, E. R.; Keinan, S.; Mori-Sánchez, P.; Contreras-García, J.; Cohen, A. J.; Yang, W. Revealing Noncovalent Interactions. J. Am. Chem. Soc. 2010, 132, 6498-6506.

(23) Liu, S. Steric Effects: a Quantitative Description from Density Functional Theory. J. Chem. Phys. 2007, 126, 244103.

(24) Deng, K.; Bensari-Bouguerra, A.; Whetstone, J.; Cohen, T. Cyclization by Intramolecular Carbolithiation of Alkyl- and Vinyllithiums Prepared by the Action of Aromatic Radical Anions on Phenyl Thioethers. High Stereoselectivity in the Cyclization Accelerated by an Allylic Lithium Oxyanion. J. Org. Chem. 2006, 71, 2360-2372.

(25) Geyer, A. G.; McClellan, W. J.; Rockway, T. W.; Stewart, K. D.; Weitzberg, M.; Wendt, M. D. Urokinase inhibitors. US6,258,822B1, 2001.

(26) Bussolari, J. C.; Rehborn, D. C. Preparation of 5-Aryl Furfurals and Aryl Thiophene-2-carboxaldehydes via Palladium-Catalyzed C-C Bond Formation in Aqueous Media. Org. Lett. 1999, 1, 965-967.

(27) Martín-Matute, B.; Nevado, C.; Cárdenas, D. J.; Echavarren, A. M. Intramolecular Reactions of Alkynes with Furans and Electron Rich Arenes Catalyzed by $\mathrm{PtCl}_{2}$ : The Role of Platinum Carbenes as Intermediates. J. Am. Chem. Soc. 2003, 125, 5757-5766.

(28) Lin, J.; Jin, X.; Bu, Y.; Cao, D.; Zhang, N.; Li, S.; Sun, Q.; Tan, C.; Gao, C.; Jiang, Y. Efficient Synthesis of RITA and its Analogues: Derivation of Analogues with Improved Antiproliferative Activity via Modulation of p53/miR-34a Pathway. Org. Biomol. Chem. 2012, 10, 9734-9746.

(29) Terai, T.; Kohno, M.; Boncompain, G.; Sugiyama, S.; Saito, N.; Fujikake, R.; Ueno, T.; Komatsu, T.; Hanaoka, K.; Okabe, T. J. Am. Chem. Soc. 2015, 137, 10464-10467.

(30) Carrettin, S.; Blanco, M. C.; Corma, A.; Hashmi, A. S. K. Artificial Ligands of Streptavidin (ALiS): Discovery, Characterization, and Application for Reversible Control of Intracellular Protein Transport. Adv. Synth. Catal. 2006, 348, 1283-1288.

(31) Feng, W.; Jiang, D.; Kee, C. W.; Liu, H.; Tan, C. H. Bicyclic Guanidine Catalyzed Asymmetric Tandem Isomerization Intramolecular-Diels-Alder Reaction: The First Catalytic Enantioselective Total Synthesis of (+)-alpha-Yohimbine. Chem. - Asian J. 2016, 11, 390-394.

(32) Hashmi, A. S. K.; Hengst, T.; Lothschütz, C.; Rominger, F. New and Easily Accessible Nitrogen Acyclic Gold(I) Carbenes: Structure and Application in the Gold-Catalyzed Phenol Synthesis as well as the Hydration of Alkynes. Adv. Synth. Catal. 2010, 352, 1315-1337.

(33) Hashmi, A. S. K.; Häffner, T.; Yang, W.; Pankajakshan, S.; Schäfer, S.; Schultes, L.; Rominger, F.; Frey, W. Gold Catalysis: NonSpirocyclic Intermediates in the Conversion of Furanynes by the Formal Insertion of an Alkyne into an Aryl-Alkyl C-C Single Bond. Chem. - Eur. J. 2012, 18, 10480-10486. 